\title{
ANALYTICAL PREDICTION OF STICK-SLIP CHAOS IN A DOUBLE SELF-EXCITED DUFFING-TYPE OSCILLATOR
}

\author{
JAN AWREJCEWICZ AND MARIUSZ HOLICKE
}

Received 10 March 2006; Accepted 21 March 2006

We have analytically predicted homoclinic bifurcations in a class of double self-excited Duffing-type oscillators using the Melnikov-Gruendler approach. Both stick-slip and smooth chaotic behaviors predicted analytically have been confirmed by numerical simulations.

Copyright (C) 2006 J. Awrejcewicz and M. Holicke. This is an open access article distributed under the Creative Commons Attribution License, which permits unrestricted use, distribution, and reproduction in any medium, provided the original work is properly cited.

\section{Introduction}

Although the Melnikov method is merely approximative, it is one of few methods, which allow to predict analytically chaos occurrence. Moreover, it can be applied to a relatively large class of dynamical systems.

To sum up, one needs to say that both the classical Melnikov method and its generalization in the form of the Melnikov-Gruendler method found their application in analysis of dynamical systems. The main advantages of both methods cover

(i) possibility of obtaining analytical results,

(ii) possibility of applying the Melnikov method in dynamical systems, whose elements are characterized by arbitrary but integrable characteristics (including discontinuous ones in a finite number of points like, e.g., friction characteristics),

(iii) high efficiency of verification of numerically generated results,

(iv) possibility of examination of strongly nonlinear systems.

Both methods are not ideal, because they have the following defects:

(i) possibility of applying only to systems, characterized by a specific phase portrait (homoclinic orbits of a critical saddle point),

(ii) each is an approximative method, which uses a small parameter,

(iii) a system, when there is no perturbation, should be integrable, 
(iv) it is possible to predict values of the parameters, causing only the so-called homoclinic chaos,

(v) complexity of mathematical calculations.

Despite the aforementioned imperfections, on the current level of science development, the Melnikov method has been accepted by the scientific community and it has been a useful tool to perform advanced investigations in nonlinear dynamics, including deterministic chaos.

Holmes and Marsden [19] applied the Melnikov method to study continuous systems of infinite degrees of freedom governed by partial differential equations.

Bulsara et al. [11] applied the Melnikov method to a system with stochastic excitation of probability distribution of finite mean value and variation. A formula, defining the Melnikov function, has been derived for a considered system in a form of probability distribution (random variable) and its mean value. The obtained analytical results were compared with numerical simulations, performed for a system of the Duffing oscillator, mathematical pendulum, and an abstract system, while high compliance of predictions and numerical experiments was achieved.

Oscillative systems are a subclass of dynamical systems. In [4] one derived formulas, determining the Melnikov function for oscillators (in particular for mechanical systems) in a simpler classical form. The proposed approach allows to simplify calculations, needed to determine conditions of homoclinic bifurcation occurrence in oscillative dynamical systems.

Taki [27] applied the Melnikov method to a bistable optical system, which is characterized by two homoclinic orbits connected with the same saddle point, while one of them is contained in the other. A criterion of the homoclinic chaos for one of the orbits was numerically determined on the basis of the formula describing the Melnikov function. A criterion corresponding to the second orbit was not considered, since it predicted chaos occurrence for relatively large values of the parameter of the considered dynamical system which should be relatively small by the assumption. The obtained results were compared with the numerical simulations and high compliance was achieved between the Melnikov method predictions of chaotic motions and the simulations.

Holmes and Marsden [19] applied the method to a periodically driven beam, which underwent buckling. By applying the Galerkin method, they obtained an equivalent mathematical model in a form of the Duffing oscillator.

Mielke and Holmes [21] applied the Melnikov method to examine a problem of buckling of a strongly curved rod. In the work, a special attention has been paid to possibility of chaos occurrence in systems, in which there are at least two heteroclinic orbits, although the Melnikov function does not possess single roots. It is possible when stable and unstable differentiable manifolds of the same saddle point (which belongs to different homoclinic orbits when there is no perturbation), as a result of appearance of perturbation and split of the homoclinic orbits, will form a chaotic attractor intersecting themselves by infinite countable number of times but they will not intersect simultaneously with other manifolds of the second equilibrium point. By the above, the Melnikov function evaluated along the heteroclinic orbit, being a measure of distance between nonintersecting manifolds, will not possess roots. Thus, it was proved that in some dynamical systems 
there exists a possibility of the chaos occurrence although the Melnikov method shows regular motions occurrence.

Moon and Li [24] considered a dependence of a fractal structure of a basin of attraction on the occurrence of homoclinic bifurcation on the example of the Duffing oscillator. One determined, with the help of the Melnikov method, a limiting value of parameters of the considered system. At these parameters, the Melnikov method predicts the occurrence of a chaotic attractor. Next, one made graphs, in which basins of attractions for several selected values of the parameters were marked. By the comparison of the obtained results, in the above described way, it follows that the structure of basin of attraction boundary stays in connection with occurrence of homoclinic bifurcation and chaos.

Moon and Holmes [22, 23] examined a system of an elastic and harmonically excited pendulum of uniformly distributed mass in a form of steel flat bar of small thickness which is located in a specific magnetic field. By applying the Galerkin method to a system, governed by partial differential equations, one obtained a discrete mathematical model of the examined system in a form of the Duffing equation. In further analysis of the examined system, one applied the classical Melnikov method, whose results were compared with laboratory investigations. One applied the classical Melnikov method to further analysis of the examined system. Its results were compared with laboratory investigations. One applied the spectral analysis to detect chaotic motions. A quantitative divergence was noted between predictions, following from the Melnikov method, and experimental investigations at qualitative compliance. Generally, a larger amplitude of external excitation turned out to be necessary to make deterministic chaos arise in the examined system than it was predicted by the Melnikov method. It follows from the cited works that a better approximation than the one of the Melnikov method was obtained by Holmes [18]. However, one needs to approach to the mentioned results critically, since one used the Fourier transform to detect chaotic motions instead of using the Lyapunov exponents. Methodological imperfections could have been a reason of incorrect identification of weak chaos as regular motions for smaller values of the external excitation, and consequently to distort the results.

Guckenheimer and Holmes [17] applied the Melnikov method to determine limiting parameters of a harmonically driven damped Duffing oscillator at which the homoclinic bifurcation occurs. One determined conditions of occurrence of subharmonic vibrations in the parameters space on the example of harmonically driven damped oscillator. Thus, the Melnikov method was applied to a system, possessing heteroclinic orbits in the phase plane (they are homoclinic orbits on a cylindric phase plane).

Koch and Leven [20] applied the Melnikov method to examine a parametrically driven mathematical pendulum. The Melnikov function and criterion of homoclinic and subharmonic bifurcation were determined for the considered system. The numerical investigations confirmed the occurrence of tangency of stable and unstable orbits for critical parameters.

In [10] the Melnikov method was used as a detector of "global homoclinic structures." One tried to find the influence on the form of frequency spectrum of time course, generated by the Duffing system. As a result of the performed investigations, one succeeded in confirming numerically the obtained linear form, of course, of the averaged spectral 
graph analytically (the Fourier integral transformation applied) for several arbitrarily selected values of the parameters.

Salam [26] used the Melnikov method to study non-Hamiltonian dissipative systems on the example of a strongly damped mathematical pendulum at relatively small timeperiodic external excitation and constant driving torque. One showed in a numerical way that the system without excitation possesses a heteroclinic orbit and its decay into stable and unstable differentiable manifolds caused by a relatively small excitation was shown. Moreover, one determined the appropriate Melnikov function. Predictions based on the Melnikov method were numerically confirmed for several selected parameters values of a given system.

Some self-excited systems with dry friction are included in a group of discontinuous systems with a delayed argument. The Melnikov method has been applied to nonsmooth systems with a delayed argument, on the example of a self-excited, relatively weak driven (quasi-autonomous) Duffing oscillator of one degree of freedom with polynomial friction [1], for the first time applied in [4]. In this work, one defined the Melnikov function for oscillators of one degree of freedom, simultaneously describing both types of motions: stick and slip. Taking into account only one criterion of chaos was a defect of this method, though one could have expected two such criteria. In [5], due to the application of the Melnikov method, one proved analytically that there can occur chaotic attractors in selfexcited autonomous systems (with zero initial excitation) with dry friction. Hence, with the help of the Melnikov method, one verified a phenomenon of deterministic chaos, known from the numerical and laboratory $[6,25]$ investigations of Galvanetto et al. [12], in self-excited systems with dry friction without external excitation in the case of one degree of freedom. The results, obtained by means of the Melnikov method, have been analytically and numerically confirmed in [3].

Among other things, on the basis of $[2,5]$, an interesting application of the Melnikov method to a rotary pin-sleeve system was presented in [7]. One considered a physicalmathematical model covering the influence of warmth, emitted during the dry friction process, on the friction force, however one performed only numerical analysis of the Melnikov function due to its "rather complex form."

The Melnikov method, despite its numerous applications in the classical form, possesses an essential defect: the method can be applied to mechanical systems of one degree of freedom with excitation. This defect is absent in the Melnikov-Gruendler method.

Guckenheimer and Holmes [17] were the first, who determined a set of parameters of relatively weak perturbed Hamiltonian system of two degrees of freedom, at which homoclinic bifurcation occurs on the basis of the KAM theorem.

Another possibility is the approach, based on the assumption that if a mechanical system of a finite number of degrees of freedom is an integrable system (possesses, e.g., cyclic coordinates), then it is described by the Routh equation [9]. If the number of first integrals allows the Routh equation to satisfy the assumptions of the Melnikov method, then it is possible to apply the classical Melnikov method to a system of a larger number of degrees of freedom. In the case when the number of first integrals is unknown, one needs to apply the Melnikov-Gruendler method [14], which is an extension of the Melnikov method to non-Hamiltonian dynamical systems of an arbitrary finite number of degrees 


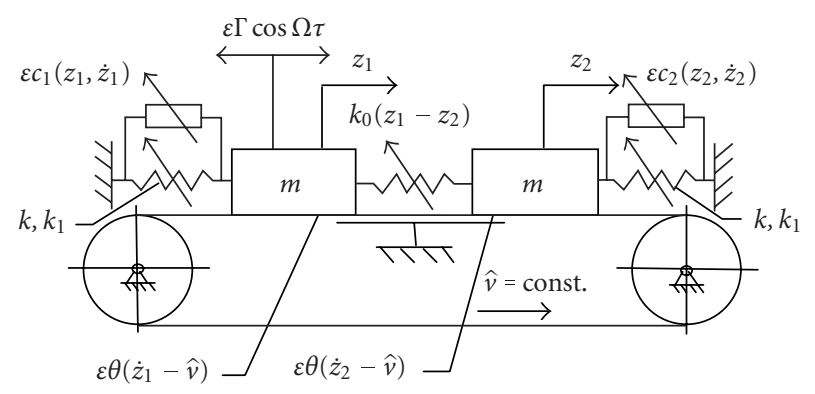

Figure 2.1. Model of the considered system.

of freedom. For some types of systems (symmetry of variational equations on a homoclinic orbit, no coupling, etc.), formulas determining chaos criteria can be simplified.

The Melnikov-Gruendler method, in contrast to the classical Melnikov method, is not widely known. It was applied in [14] to consider small vibrations of a spherical pendulum subjected to magnetic field with a relatively small and then large viscous damping (nonHamiltonian system). Moreover, one applied the Melnikov-Gruendler method to several possessing physical interpretation and abstract dynamical systems $[15,16]$, in order to detect deterministic chaos and analyze structural changes of homoclinic orbits.

The reference [5] played a key role during the attempt to apply the Melnikov-Gruendler method to self-excited systems with polynomial friction of two degrees of freedom [8], however the obtained results did not reflect complexity of the multidimensional problem entirely, by making use of only a few percents of the Melnikov-Gruendler method capabilities. By this reason, one can say that the problem of homoclinic chaos occurrence in two-degree-of-freedom systems with dry friction has not been examined by means of the Melnikov-Gruendler method so far.

\section{Analytical prediction of chaos}

A Duffing-type 2-DOF mechanical system with discontinuities is analyzed (see Figure 2.1 ). It consists of two masses $m$ lying on a rigid belt moving at constant speed $\hat{v}$. It is assumed that between both masses the belt polynomial-type friction occurs. Masses $m$ are coupled by springs with stiffness satisfying the following conditions: $k_{0}(-z)=-k_{0}(z)$. Each of the masses is linked to a basis by nonlinear elastic elements of Duffing-type characteristics as well as elements modeling internal aerodynamic and hydrodynamic friction. One of the masses is driven harmonically with frequency $\Omega$ and relatively small amplitude $\Gamma$. It is assumed that all frictional forces are relatively small, which is formally exhibited by an introduction of small (perturbation) parameter $\varepsilon$.

Dimensional equations of motion have the form

$$
\begin{gathered}
m \ddot{z}_{1}-k \cdot z_{1}+k_{1} \cdot z_{1}^{3}+\varepsilon c_{1}\left(z_{1}, \dot{z}_{1}\right)+k_{0}\left(z_{1}-z_{2}\right)+\varepsilon \theta\left(\dot{z}_{1}-\hat{v}\right)=\varepsilon \Gamma \cos \Omega \tau, \\
m \ddot{z}_{2}-k \cdot z_{2}+k_{1} \cdot z_{2}^{3}+\varepsilon c_{2}\left(z_{2}, \dot{z}_{2}\right)-k_{0}\left(z_{1}-z_{2}\right)+\varepsilon \theta\left(\dot{z}_{2}-\hat{v}\right)=0,
\end{gathered}
$$


6 Stick-slip chaos in a self-excited oscillator

where dry friction characteristic is as follows:

$$
\theta(\dot{z}-\hat{v})=\theta_{0} \operatorname{sgn}(\dot{z}-\hat{v})-\hat{\alpha}(\dot{z}-\hat{v})+\hat{\beta}(\dot{z}-\hat{v})^{3}
$$

Elements modeling internal aerodynamic and hydrodynamic friction are defined by the formula $c_{j}\left(z_{j}, \dot{z}_{j}\right)=k_{j 1} z_{j}+c_{j 1} \dot{z}_{j}+c_{j 2} \dot{z}_{j}^{2} \operatorname{sgn} \dot{z}_{j}+c_{j 3} \dot{z}_{j}^{3}, j=1,2$. According to the introduced general form, one may simultaneously analyze oscillations of various 2-DOF mechanical systems. This is easily realized by a proper choice of parameters. For example, in order to study a self-excited 2-DOF Duffing-type oscillator with polynomial-type friction, one has to assign nonzero values of $\theta_{0}, \hat{\alpha}, \hat{\beta}$, whereas $k_{j 1}, c_{j 1}, c_{j 2}, c_{j 3}$ should be equal to zero. On the other hand, taking $\theta_{0}, \hat{\alpha}, \hat{\beta}, k_{j 1}, c_{j 1}, c_{j 3}$ equal to zero and assuming $c_{j 2} \neq 0$, it is possible to predict thresholds of chaos in our 2-DOF mechanical system with aerodynamic friction, and so forth. Since the studied system has two degrees of freedom, the Melnikov-Gruendler approach is applied to study homoclinic chaos occurrence.

Introducing the variables

$$
z_{1}=x_{1} \sqrt{\frac{k}{k_{1}}}, \quad z_{2}=x_{2} \sqrt{\frac{k}{k_{1}}}, \quad \tau=t \sqrt{\frac{m}{k_{1}}},
$$

the following nondimensional equations are obtained:

$$
\begin{gathered}
\ddot{x}_{1}-x_{1}+x_{1}^{3}+a \cdot k_{0}\left(b \cdot\left(x_{1}-x_{2}\right)\right)=\varepsilon\left(\gamma \cos \omega t-\delta_{1}\left(x_{1}, \dot{x}_{1}\right)-T\left(\dot{x}_{1}-v_{*}\right)\right), \\
\ddot{x}_{2}-x_{2}+x_{2}^{3}-a \cdot k_{0}\left(b \cdot\left(x_{1}-x_{2}\right)\right)=-\varepsilon\left(T\left(\dot{x}_{2}-v_{*}\right)+\delta_{2}\left(x_{2}, \dot{x}_{2}\right)\right),
\end{gathered}
$$

where

$$
\begin{gathered}
T\left(\dot{x}-v_{*}\right)=T_{0} \operatorname{sgn}\left(\dot{x}-v_{*}\right)-\alpha\left(\dot{x}-v_{*}\right)+\beta\left(\dot{x}-v_{*}\right)^{3}, \\
\delta_{j}\left(x_{j}, \dot{x}_{j}\right)=\alpha_{j 1} x_{j}+\delta_{j 1} \dot{x}_{j}+\delta_{j 2} \dot{x}_{j}^{2} \operatorname{sgn}\left(\dot{x}_{j}\right)+\delta_{j 3} \dot{x}_{j}^{3} .
\end{gathered}
$$

In the above, the following notation is applied: $a=(1 / k) \sqrt{k_{1} / k}, b=\sqrt{k / k_{1}}, \gamma=\Gamma\left(\sqrt{k_{1}} / k\right)$, $\omega=\Omega \sqrt{m / k}$ and $v_{*}=\hat{v} \sqrt{m k_{1}} / k, T_{0}=\left(\theta_{0} / k\right) \sqrt{k_{1} / k}, \alpha=\hat{\alpha} \sqrt{m k}, \beta=\hat{\beta} k^{2} / m k_{1} \sqrt{m k}, \alpha_{j 1}=$ $k_{j 1} / \sqrt{m k}, \delta_{j 1}=c_{j 1} / \sqrt{m k}, \delta_{j 2}=\left(c_{j 2} / m\right) \sqrt{k / k_{1}}, \delta_{j 3}=\left(c_{j 3} / k_{1}\right)(k / m)^{3 / 2}$.

The analyzed system is given in the first-order ODEs form

$$
\begin{gathered}
\dot{x}_{1}=v_{1}, \\
\dot{v}_{1}=x_{1}-x_{1}^{3}-a \cdot k_{0}\left(b \cdot\left(x_{1}-x_{2}\right)\right)+\varepsilon\left(\gamma \cos \omega t-\delta_{1}\left(x_{1}, v_{1}\right)-T\left(v_{1}-v_{*}\right)\right), \\
\dot{x}_{2}=v_{2}, \\
\dot{v}_{2}=x_{2}-x_{2}^{3}+a \cdot k_{0}\left(b \cdot\left(x_{1}-x_{2}\right)\right)-\varepsilon\left(T\left(v_{2}-v_{*}\right)+\delta_{2}\left(x_{2}, v_{2}\right)\right) .
\end{gathered}
$$

For $\varepsilon=0$, one gets

$$
\begin{aligned}
& \ddot{x}_{1}-x_{1}+x_{1}^{3}+a \cdot k_{0}\left(b \cdot\left(x_{1}-x_{2}\right)\right)=0, \\
& \ddot{x}_{2}-x_{2}+x_{2}^{3}-a \cdot k_{0}\left(b \cdot\left(x_{1}-x_{2}\right)\right)=0,
\end{aligned}
$$


or equivalently

$$
\begin{gathered}
\dot{x}_{1}=v_{1}, \\
\dot{v}_{1}=x_{1}-x_{1}^{3}-a \cdot k_{0}\left(b \cdot\left(x_{1}-x_{2}\right)\right), \\
\dot{x}_{2}=v_{2}, \\
\dot{v}_{2}=x_{2}-x_{2}^{3}+a \cdot k_{0}\left(b \cdot\left(x_{1}-x_{2}\right)\right) .
\end{gathered}
$$

Since the analyzed system has an equilibrium in the origin of phase coordinates, the following homoclinic solution is assumed:

$$
q_{0}\left(x_{1}, x_{2}\right)(t)=(A r(B t), A r(B t))
$$

where $r(t)=\operatorname{sech}(t)$. Constants $A$ and $B$ are defined by a substitution of the homoclinic orbit into (2.8) from the following algebraic equation:

$$
\operatorname{sech}(B t)\left(1-B^{2}+\left(A^{2}-2 B^{2}\right) \operatorname{sech}^{2}(B t)\right)=0 .
$$

Assuming $B=1$ and substituting it into (2.11), one obtains

$$
A= \pm \sqrt{2}
$$

Furthermore, we assume that the critical point being the origin of our coordinates system is associated with a homoclinic solution of the form

$$
q_{0}\left(x_{1}, x_{2}\right)(t)=(A r(B t),-A r(B t))
$$

Constants $A$ and $B$ are determined in a way similar to the previous one (substituting the homoclinic orbit equation into (2.8)):

$$
a \cdot k_{0}(2 A b \operatorname{sech}(B t))+A \operatorname{sech}(B t)\left(B^{2}-1+\left(A^{2}-2 B^{2}\right) \operatorname{sech}^{2}(B t)\right)=0 .
$$

Since this equation is satisfied for $k_{0}(z)=k_{0} \cdot z$, hence

$$
A \operatorname{sech}(B t)\left(B^{2}-1+2 a k_{0} b+\left(A^{2}-2 B^{2}\right) \operatorname{sech}^{2}(B t)\right)=0 .
$$

Taking $B= \pm \sqrt{1-2 a b k_{0}}\left(a b k_{0}<1 / 2\right)$, one gets

$$
A^{2}-2\left(1-2 a b k_{0}\right)=0,
$$

and finally

$$
A= \pm \sqrt{2\left(1-2 a b k_{0}\right)}, \quad a b k_{0}<\frac{1}{2}
$$


8 Stick-slip chaos in a self-excited oscillator

Note that irrespective of the coupling characteristic $k_{0}\left(z_{1}-z_{2}\right)$ there are two homoclinic orbits associated with the considered equilibrium and defined by the formula

$$
q_{01,2}\left(x_{1}, \dot{x}_{1}, x_{2}, \dot{x}_{2}\right)(t)=\left(\begin{array}{c} 
\pm \sqrt{2} r(t) \\
\pm \sqrt{2} \dot{r}(t) \\
\pm \sqrt{2} r(t) \\
\pm \sqrt{2} \dot{r}(t)
\end{array}\right) .
$$

Furthermore, for stiffness of the coupling element defined by formula $k_{0}(z)=k_{0} \cdot z$, two further homoclinic orbits occur for $a b k_{0}<1 / 2$ defined as follows:

$$
q_{03,4}\left(x_{1}, \dot{x}_{1}, x_{2}, \dot{x}_{2}\right)(t)=\left(\begin{array}{c} 
\pm \sqrt{2\left(1-2 a b k_{0}\right)} r\left(\sqrt{1-2 a b k_{0}} t\right) \\
\pm \sqrt{2\left(1-2 a b k_{0}\right)} \dot{r}\left(\sqrt{1-2 a b k_{0}} t\right) \\
\mp \sqrt{2\left(1-2 a b k_{0}\right)} r\left(\sqrt{1-2 a b k_{0}} t\right) \\
\mp \sqrt{2\left(1-2 a b k_{0}\right)} \dot{r}\left(\sqrt{1-2 a b k_{0}} t\right)
\end{array}\right), \quad a b k_{0}<\frac{1}{2}
$$

Next, we compute the quantities necessary for direct application of the MelnikovGruendler method. Linearization of system (2.8) in the vicinity of a saddle-type equilibrium yields

$$
\begin{gathered}
\ddot{x}_{1}+\left(-1+3 x_{10}^{2}+\left.a \frac{d k_{0}\left(b \cdot\left(x_{1}-x_{2}\right)\right)}{d x_{1}}\right|_{x_{1}=x_{10}, x_{2}=x_{20}}\right) x_{1} \\
+\left.a \frac{d k_{0}\left(b \cdot\left(x_{1}-x_{2}\right)\right)}{d x_{2}}\right|_{x_{1}=x_{10}, x_{2}=x_{20}} \cdot x_{2}=0, \\
\ddot{x}_{2}+\left(-1+3 x_{20}^{2}-\left.a \frac{d k_{0}\left(b \cdot\left(x_{1}-x_{2}\right)\right)}{d x_{2}}\right|_{x_{1}=x_{10}, x_{2}=x_{20}}\right) x_{2} \\
-\left.a \frac{d k_{0}\left(b \cdot\left(x_{1}-x_{2}\right)\right)}{d x_{1}}\right|_{x_{1}=x_{10}, x_{2}=x_{20}} \cdot x_{1}=0 .
\end{gathered}
$$

The linearized (variational) motion equations along two orbits (2.18) are defined by the formulas

$$
\begin{aligned}
& \ddot{x}_{1}+\left(6 \operatorname{sech}^{2} t-1+c\right) x_{1}-c x_{2}=0, \\
& \ddot{x}_{2}+\left(6 \operatorname{sech}^{2} t-1+c\right) x_{2}-c x_{1}=0
\end{aligned}
$$

where

$$
c=\left.a \frac{d k_{0}\left(b \cdot\left(x_{1}-x_{2}\right)\right)}{d x_{1}}\right|_{x_{1}=x_{2}}=-\left.a \frac{d k_{0}\left(b \cdot\left(x_{1}-x_{2}\right)\right)}{d x_{2}}\right|_{x_{1}=x_{2}},
$$


or in the equivalent form

$$
\begin{gathered}
\dot{x}_{1}=v_{1}, \\
\dot{v}_{1}=-\left(6 \operatorname{sech}^{2} t-1+c\right) x_{1}+c x_{2}, \\
\dot{x}_{2}=v_{2}, \\
\dot{v}_{2}=-\left(6 \operatorname{sech}^{2} t-1+c\right) x_{2}+c x_{1} .
\end{gathered}
$$

Subtraction of (2.21) gives

$$
\ddot{y}+\left(6 \operatorname{sech}^{2} t-\bar{b}^{2}\right) y=0,
$$

where $y=x_{1}-x_{2}, \bar{b}^{2}=1-2 c$. Note that the obtained second-order differential equation has time-dependent coefficient. In order to solve it, the following theorem is applied [13]. Theorem 2.1. Let $u=u(t)$ be a general solution to the associated equation

$$
\frac{d^{2} u}{d t^{2}}=[g(t)+h] u
$$

and let $f=f(t)$ be a particular solution to the above equation for a certain $h=h_{1}$, then a generalize solution of the linear homogeneous second-order ODE

$$
\frac{d^{2} y}{d t^{2}}=\left[f(t) \frac{d^{2}}{d t^{2}}\left(\frac{1}{f(t)}\right)+h+h_{1}\right] y
$$

is given by the formula

$$
y=u^{\prime}(t)-u(t) \frac{f^{\prime}(t)}{f(t)}
$$

for $h \neq h_{1}$.

Taking $\left(g(t)=-2 \operatorname{sech}^{2} t, h_{1}=4, h=\bar{b}^{2}, f(t)=\cosh ^{2} t\right)$, the associated equation is defined as follows:

$$
\ddot{u}+\left(2 \operatorname{sech}^{2} t-\bar{b}^{2}\right) u=0,
$$

which has also time-dependent coefficient. In order to solve it again, Theorem 2.1 is applied (we take now $g(t) \equiv 0, h_{1}=1, h=\bar{b}^{2}, f(t)=\cosh t$ ). Solving the obtained equation of the form

$$
\frac{d^{2} \bar{u}}{d t^{2}}=\bar{b}^{2} \bar{u}
$$

one gets

$$
\bar{u}=C_{3} e^{\bar{b} t}-C_{4} e^{-\bar{b} t}
$$


10 Stick-slip chaos in a self-excited oscillator

where $C_{3}$ and $C_{4}$ are the constants of integrations. A general solution of adjoint equation is

$$
u=C_{3} e^{\bar{b} t}(b t-\operatorname{tgh} t)-C_{4} e^{-\bar{b} t}(b t+\operatorname{tgh} t)
$$

and hence

$$
\begin{aligned}
y= & C_{1} e^{\bar{b} t}\left(2+\bar{b}^{2}-3 \operatorname{sech}^{2} t-3 \bar{b} \operatorname{tgh} t\right) \\
& -C_{2} e^{-\bar{b} t}\left(2+\bar{b}^{2}-3 \operatorname{sech}^{2} t+3 \bar{b} \operatorname{tgh} t\right) .
\end{aligned}
$$

Since $\bar{b}^{2}=1-2 c$, the following general solution of (2.24) is obtained:

$$
\begin{aligned}
y(t)= & C_{3} y_{s 3}+C_{4} y_{s 4} \\
= & C_{3} e^{\sqrt{1-2 c} t}\left(-\frac{2}{3} c-(\sqrt{1-2 c}-\operatorname{tgh} t) \operatorname{tgh} t\right) \\
& +C_{4} e^{-\sqrt{1-2 c} t}\left(-\frac{2}{3} c+(\sqrt{1-2 c}+\operatorname{tgh} t) \operatorname{tgh} t\right),
\end{aligned}
$$

where $y_{s 3}$ and $y_{s 4}$ are the particular solutions.

Summation of (2.21) gives

$$
\ddot{x}+\left(6 \operatorname{sech}^{2} t-1\right) x=0,
$$

where $x=x_{1}+x_{2}$. Observe that this is a particular case of (2.24) for $c=0$. Using (2.33), the following solution is obtained:

$$
x(t)=C \dot{r}(t)
$$

where $C$ denotes the constant of integration. The second linearly independent integral is found using Lagrange's method of constant variation. Assuming

$$
x(t)=C(t) \dot{r}(t)
$$

and substituting the obtained formulas into (2.34), one gets

$$
2 \dot{C} \ddot{r}+\ddot{C} \dot{r}=-C \dddot{r}-\left(6 \operatorname{sech}^{2} t-1\right) C \dot{r}=\ddot{x}+\left(6 \operatorname{sech}^{2} t-1\right) x .
$$

Owing to (2.34), the following equation is obtained:

$$
2 \dot{C} \ddot{r}+\ddot{C} \dot{r}=0,
$$

which has the following general solution:

$$
C(t)=C_{2}+C_{1}\left(\frac{3}{2} t-\operatorname{ctgh} t+\frac{1}{4} \sinh 2 t\right),
$$


where $C_{1}$ and $C_{2}$ are the integration constants. Finally, a general solution of (2.34) is as follows:

$$
x(t)=C_{1} x_{s 1}+C_{2} x_{s 2}=C_{1}\left(\frac{3}{2} t-\operatorname{ctgh} t+\frac{1}{4} \sinh 2 t\right) \operatorname{sech} t \operatorname{tgh} t+C_{2} \operatorname{sech} t \operatorname{tgh} t .
$$

Since $y=x_{1}-x_{2}$ and $x=x_{1}+x_{2}$, a solution to (2.21) is a linear combination defined by the following formulas:

$$
x_{1}=\frac{1}{2}(x+y), \quad x_{2}=\frac{1}{2}(x-y) .
$$

System (2.21) has the following fundamental matrix of solutions:

$$
\begin{aligned}
\gamma(t) & =\left[\begin{array}{cccc}
y_{s 1} & x_{s 1} & y_{s 2} & x_{s 2} \\
\dot{y}_{s 1} & \dot{x}_{s 1} & \dot{y}_{s 2} & \dot{x}_{s 2} \\
-y_{s 1} & x_{s 1} & -y_{s 2} & x_{s 2} \\
-\dot{y}_{s 1} & \dot{x}_{s 1} & -\dot{y}_{s 2} & \dot{x}_{s 2}
\end{array}\right] \\
= & {\left[\begin{array}{cccc}
P(t) \exp (\sqrt{1-2 c} t) & Q(t) \dot{r}(t) & P(-t) \exp (-\sqrt{1-2 c} t) & \dot{r}(t) \\
(P(t) \exp (\sqrt{1-2 c} t))^{\bullet} & (Q(t) \dot{r}(t))^{\bullet} & (P(-t) \exp (-\sqrt{1-2 c} t)) & \ddot{r}(t) \\
-P(t) \exp (\sqrt{1-2 c} t) & Q(t) \dot{r}(t) & -P(-t) \exp (-\sqrt{1-2 c} t) & \dot{r}(t) \\
-(P(t) \exp (\sqrt{1-2 c} t))^{\bullet} & (Q(t) \dot{r}(t))^{\bullet} & -(P(-t) \exp (-\sqrt{1-2 c} t))^{\bullet} & \ddot{r}(t)
\end{array}\right], }
\end{aligned}
$$

where $Q(t)=(3 / 2) t-\operatorname{ctgh} t+(1 / 4) \sinh 2 t$ and $P(t)=-(2 / 3) c-(\sqrt{1-2 c}-\operatorname{tgh} t) \operatorname{tgh} t$.

Linearized (variational) equations of motion along both orbits (2.19) are governed by the following equations:

$$
\begin{aligned}
& \ddot{x}_{1}+\left(6(1-2 c) \operatorname{sech}^{2}(\sqrt{1-2 c} t)+c-1\right) x_{1}-c x_{2}=0, \\
& \ddot{x}_{2}+\left(6(1-2 c) \operatorname{sech}^{2}(\sqrt{1-2 c} t)+c-1\right) x_{2}-c x_{1}=0,
\end{aligned}
$$

where $c=a b k_{0}=k_{0} / k<1 / 2$. The equivalent first-order ODEs are

$$
\begin{gathered}
\dot{x}_{1}=v_{1}, \\
\dot{v}_{1}=-\left(6(1-2 c) \operatorname{sech}^{2}(\sqrt{1-2 c} t)-1+c\right) x_{1}+c x_{2}, \\
\dot{x}_{2}=v_{2}, \\
\dot{v}_{2}=-\left(6(1-2 c) \operatorname{sech}^{2}(\sqrt{1-2 c} t)-1+c\right) x_{2}+c x_{1} .
\end{gathered}
$$

Solving (2.43) and after summing them, one obtains

$$
\ddot{x}+\left(6(1-2 c) \operatorname{sech}^{2}(\sqrt{1-2 c} t)-1\right) x=0,
$$


12 Stick-slip chaos in a self-excited oscillator

where $x=x_{1}+x_{2}$. Owing to introduction of a new independent variable by formula $\chi=$ $\sqrt{1-2 c} t,\left(\ddot{x}=x^{\prime \prime} \dot{\chi}^{2}\right)$, one has

$$
x^{\prime \prime}+\left(6 \operatorname{sech}^{2} \chi-\frac{1}{1-2 c}\right) x=0
$$

where $x^{\prime \prime}=d^{2} x / d \chi^{2}$.

Note that the obtained equation is analogous to (2.24) assuming $b^{-2}=1-2 c$. Applying (2.32), one obtains

$$
\begin{aligned}
x(\chi)= & 3 C_{1} e^{\chi / \sqrt{1-2 c}}\left(\frac{3-4 c}{3(1-2 c)}-\operatorname{sech}^{2} \chi-\frac{\operatorname{tgh} \chi}{\sqrt{1-2 c}}\right) \\
& +3 C_{2} e^{-\chi / \sqrt{1-2 c}}\left(\frac{3-4 c}{3(1-2 c)}-\operatorname{sech}^{2} \chi+\frac{\operatorname{tgh} \chi}{\sqrt{1-2 c}}\right) .
\end{aligned}
$$

Since $\chi=\sqrt{1-2 c} t$, a solution to (2.45) is given by the formula

$$
\begin{aligned}
x(t)= & C_{1} x_{s 1}+C_{2} x_{s 2} \\
= & C_{1} e^{t}\left(\frac{3-4 c}{3(1-2 c)}-\operatorname{sech}^{2}(\sqrt{1-2 c} t)-\frac{\operatorname{tgh}(\sqrt{1-2 c} t)}{\sqrt{1-2 c}}\right) \\
& +C_{2} e^{-t}\left(\frac{3-4 c}{3(1-2 c)}-\operatorname{sech}^{2}(\sqrt{1-2 c} t)+\frac{\operatorname{tgh}(\sqrt{1-2 c} t)}{\sqrt{1-2 c}}\right) .
\end{aligned}
$$

Subtraction of both sides of (2.43) yields

$$
\ddot{y}+\left(6(1-2 c) \operatorname{sech}^{2}(\sqrt{1-2 c} t)-1+2 c\right) y=0,
$$

where $y=x_{1}-x_{2}$. Changing the independent variable by formula $\chi=\sqrt{1-2 c t}$, one obtains

$$
y^{\prime \prime}+\left(6 \operatorname{sech}^{2} \chi-1\right) y=0
$$

where $y^{\prime \prime}=d^{2} y / d \chi^{2}$.

Observe that an analogous equation is governed by (2.34). Using (2.40) and taking into account $\chi=\sqrt{1-2 c} t$, we have

$$
\begin{aligned}
y(t)= & C_{1} y_{s 1}+C_{2} y_{s 2} \\
= & C_{1}\left(\frac{3}{2} \sqrt{1-2 c} t-\operatorname{ctgh} \sqrt{1-2 c} t+\frac{1}{4} \sinh (2 \sqrt{1-2 c} t)\right) \operatorname{sech}(\sqrt{1-2 c} t) \operatorname{tgh}(\sqrt{1-2 c} t) \\
& +C_{2} \operatorname{sech}(\sqrt{1-2 c} t) \operatorname{tgh}(\sqrt{1-2 c} t) .
\end{aligned}
$$


Since $y=x_{1}-x_{2}$ and $x=x_{1}+x_{2}$ are also solutions, a solution of system (2.43) is their linear combination, that is,

$$
x_{1}=\frac{1}{2}(x+y), \quad x_{2}=\frac{1}{2}(x-y) .
$$

The system of (2.44) has the fundamental matrix defined as

$$
\begin{aligned}
\gamma(t) & =\left[\begin{array}{cccc}
y_{s 1} & x_{s 1} & y_{s 2} & x_{s 2} \\
\dot{y}_{s 1} & \dot{x}_{s 1} & \dot{y}_{s 2} & \dot{x}_{s 2} \\
-y_{s 1} & x_{s 1} & -y_{s 2} & x_{s 2} \\
-\dot{y}_{s 1} & \dot{x}_{s 1} & -\dot{y}_{s 2} & \dot{x}_{s 2}
\end{array}\right] \\
& =\left[\begin{array}{cccc}
e^{t} R(\sqrt{1-2 c} t) & Q(\sqrt{1-2 c} t) \dot{r}(\sqrt{1-2 c} t) & e^{-t} R(-\sqrt{1-2 c} t) & \dot{r}(\sqrt{1-2 c} t) \\
\left(e^{t} R(\sqrt{1-2 c} t)\right)^{\bullet} & (Q(\sqrt{1-2 c} t) \dot{r}(\sqrt{1-2 c} t))^{\bullet} & \left(e^{-t} R(-\sqrt{1-2 c} t)\right)^{\bullet} & \ddot{r}(\sqrt{1-2 c} t) \\
-e^{t} R(\sqrt{1-2 c} t) & Q(\sqrt{1-2 c} t) \dot{r}(\sqrt{1-2 c} t) & -e^{-t} R(-\sqrt{1-2 c} t) & \dot{r}(\sqrt{1-2 c} t) \\
-\left(e^{t} R(\sqrt{1-2 c} t)\right)^{\bullet} & (Q(\sqrt{1-2 c} t) \dot{r}(\sqrt{1-2 c} t))^{\bullet} & -\left(e^{-t} R(-\sqrt{1-2 c} t)\right)^{\bullet} & \ddot{r}(\sqrt{1-2 c} t)
\end{array}\right],
\end{aligned}
$$

where $Q(t)=(3 / 2) t-\operatorname{ctgh} t+(1 / 4) \sinh 2 t$ and $R(t)=(3-4 c) / 3(1-2 c)-\operatorname{sech}^{2}(t)-$ $\operatorname{tgh}(t) / \sqrt{1-2 c}$.

Next, we consider system (2.4) for $\varepsilon>0$. A perturbation vector associated with homoclinic orbits (2.18) and sign " + " is as follows:

$$
h\left(q_{01}, t\right)=\left[\begin{array}{c}
0 \\
\gamma \cos \omega t-\delta_{1}\left(x_{10}, \dot{x}_{10}\right)-T\left(\dot{x}_{01}-v_{*}\right) \\
0 \\
-T\left(\dot{x}_{02}-v_{*}\right)-\delta_{2}\left(x_{20}, \dot{x}_{20}\right)
\end{array}\right]
$$

Substituting the first column of the fundamental solution matrix (2.42) by vector $h\left(q_{01}, t\right)$, one obtains the matrix whose determinant is

$K_{1}\left(t, t_{0}\right)$

$$
=\left|\begin{array}{cccc}
0 & Q(t) \dot{r}(t) & P(-t) \exp (-\sqrt{1-2 c} t) & \dot{r}(t) \\
\gamma \cos \omega\left(t+t_{0}\right)-\delta_{1}\left(x_{10}, \dot{x}_{10}\right)-T\left(\dot{x}_{01}-v_{*}\right) & (Q(t) \dot{r}(t))^{\bullet} & (P(-t) \exp (-\sqrt{1-2 c} t))^{\bullet} & \ddot{r}(t) \\
0 & Q(t) \dot{r}(t) & -P(-t) \exp (-\sqrt{1-2 c} t) & \dot{r}(t) \\
-T\left(\dot{x}_{02}-v_{*}\right)-\delta_{2}\left(x_{20}, \dot{x}_{20}\right) & (Q(t) \dot{r}(t))^{\bullet} & -(P(-t) \exp (-\sqrt{1-2 c} t))^{\bullet} & \ddot{r}(t)
\end{array}\right| .
$$


14 Stick-slip chaos in a self-excited oscillator

Its development with respect to the first column gives

$$
\begin{aligned}
K_{1}\left(t, t_{0}\right)= & 2 P(-t) \exp (-\sqrt{1-2 c} t) \\
& \times\left(\gamma \cos \omega\left(t+t_{0}\right)-\delta_{1}\left(x_{10}, \dot{x}_{10}\right)+\delta_{2}\left(x_{20}, \dot{x}_{20}\right)-T\left(\dot{x}_{01}-v_{*}\right)+T\left(\dot{x}_{02}-v_{*}\right)\right) .
\end{aligned}
$$

Since in the considered case $x_{01}=x_{02}$, one gets

$$
K_{1}\left(t, t_{0}\right)=2 P(-t) \exp (-\sqrt{1-2 c} t)\left(\gamma \cos \omega\left(t+t_{0}\right)-\delta_{1}\left(x_{10}, \dot{x}_{10}\right)+\delta_{2}\left(x_{10}, \dot{x}_{10}\right)\right) .
$$

Substitution of the second column of the fundamental matrix solution (2.42) by vector $h\left(q_{01}, t\right)$ gives a matrix whose determinant is

$$
\begin{aligned}
& K_{2}\left(t, t_{0}\right) \\
& =\left|\begin{array}{cccr}
P(t) \exp (D) & 0 & P(-t) \exp (-D) & \dot{r}(t) \\
(P(t) \exp (D))^{\bullet} & \gamma \cos \omega\left(t+t_{0}\right)-\delta_{1}\left(x_{10}, \dot{x}_{10}\right)-T\left(\dot{x}_{01}-v_{*}\right) & (P(-t) \exp (-D))^{\bullet} & \ddot{r}(t) \\
-P(t) \exp (D) & 0 & -P(-t) \exp (-D) & \dot{r}(t) \\
-(P(t) \exp (D))^{\bullet} & -T\left(\dot{x}_{02}-v_{*}\right)-\delta_{2}\left(x_{20}, \dot{x}_{20}\right) & -(P(-t) \exp (-D))^{\bullet} & \ddot{r}(t)
\end{array}\right|, \\
& \text { Its development with respect to the second column gives } \\
& K_{2}\left(t, t_{0}\right)=2 A\left(\gamma \cos \omega\left(t+t_{0}\right)-\delta_{1}\left(x_{10}, \dot{x}_{10}\right)\right. \\
& \left.-\delta_{2}\left(x_{20}, \dot{x}_{20}\right)-T\left(\dot{x}_{01}-v_{*}\right)-T\left(\dot{x}_{02}-v_{*}\right)\right) \dot{r}(t),
\end{aligned}
$$$$
\text { where } D=\sqrt{1-2 c} t \text {. }
$$

where (see Theorem 2.1)

$$
A=\left|\begin{array}{cc}
P(t) \exp (\sqrt{1+2 a} t) & P(-t) \exp (-\sqrt{1-2 a} t) \\
(P(t) \exp (\sqrt{1+2 a} t))^{\bullet} & (P(-t) \exp (-\sqrt{1-2 a} t))^{\bullet}
\end{array}\right|=\text { const. }
$$

Since $x_{01}=x_{02}$, one gets

$$
K_{2}\left(t, t_{0}\right)=2 A\left(\gamma \cos \omega\left(t+t_{0}\right)-\delta_{1}\left(x_{10}, \dot{x}_{10}\right)-\delta_{2}\left(x_{10}, \dot{x}_{10}\right)-2 T\left(\dot{x}_{01}-v_{*}\right)\right) \dot{r}(t) .
$$

Substituting the third column of the fundamental solution matrix (2.42) by vector $h\left(q_{01}, t\right)$, one obtains a matrix whose determinant is

$$
\begin{aligned}
& K_{3}\left(t, t_{0}\right) \\
& =\left|\begin{array}{cccc}
P(t) \exp (\sqrt{1-2 c} t) & Q(t) \dot{r}(t) & 0 & \dot{r}(t) \\
(P(t) \exp (\sqrt{1-2 c} t))^{\bullet} & (Q(t) \dot{r}(t))^{\bullet} & \phi o s \omega\left(t+t_{0}\right)-\delta_{1}\left(x_{10}, \dot{x}_{10}\right)-T\left(\dot{x}_{01}-v_{*}\right) & \ddot{r}(t) \\
-P(t) \exp (\sqrt{1-2 c} t) & Q(t) \dot{r}(t) & 0 & \dot{r}(t) \\
-(P(t) \exp (\sqrt{1-2 c} t))^{\bullet} & (Q(t) \dot{r}(t))^{\bullet} & -T\left(\dot{x}_{02}-v_{*}\right)-\delta_{2}\left(x_{20}, \dot{x}_{20}\right) & \ddot{r}(t)
\end{array}\right| .
\end{aligned}
$$


Development with respect to the third column provides

$$
\begin{aligned}
K_{3}\left(t, t_{0}\right)= & -2 P(t) \exp (\sqrt{1-2 c} t) \\
& \times\left(\gamma \cos \omega\left(t+t_{0}\right)-\delta_{1}\left(x_{10}, \dot{x}_{10}\right)-\delta_{2}\left(x_{20}, \dot{x}_{20}\right)-T\left(\dot{x}_{01}-v_{*}\right)-T\left(\dot{x}_{02}-v_{*}\right)\right) .
\end{aligned}
$$

Since $x_{01}=x_{02}$, one obtains

$$
\begin{aligned}
K_{3}\left(t, t_{0}\right)=-2 & \gamma \cos \omega\left(t+t_{0}\right)-\delta_{1}\left(x_{10}, \dot{x}_{10}\right) \\
& \left.-\delta_{2}\left(x_{10}, \dot{x}_{10}\right)-2 T\left(\dot{x}_{01}-v_{*}\right)\right) P(t) \exp (\sqrt{1-2 c} t) .
\end{aligned}
$$

Substituting the fourth column of the fundamental solution matrix (2.42) by vector $h\left(q_{01}, t\right)$, a new matrix is obtained, whose determinant is as follows:

$K_{4}\left(t, t_{0}\right)$

$=\left|\begin{array}{cccc}P(t) \exp (D) & Q(t) \dot{r}(t) & P(-t) \exp (-D) & 0 \\ (P(t) \exp (D))^{\bullet} & (Q(t) \dot{r}(t))^{\bullet} & (P(-t) \exp (-D))^{\bullet} \gamma \cos \omega\left(t+t_{0}\right)-\delta_{1}\left(x_{10}, \dot{x}_{10}\right)-T\left(\dot{x}_{01}-v_{*}\right) \\ -P(t) \exp (D) & Q(t) \dot{r}(t) & -P(-t) \exp (-D) & 0 \\ -(P(t) \exp (D))^{\bullet} & (Q(t) \dot{r}(t))^{\bullet} & -(P(-t) \exp (-D))^{\bullet} & -T\left(\dot{x}_{02}-v_{*}\right)-\delta_{2}\left(x_{20}, \dot{x}_{20}\right)\end{array}\right|$,

where $D=\sqrt{1-2 c} t$.

Development with respect to the fourth column gives $\left|\lim _{t \rightarrow \pm \infty} K_{4}\left(t, t_{0}\right)\right|=\infty$.

A similar procedure is applied for sign "-", where the perturbation vector is as follows:

$$
h\left(q_{02}, t\right)=\left[\begin{array}{c}
0 \\
\gamma \cos \omega t+\delta_{1}\left(x_{10}, \dot{x}_{10}\right)-T\left(-\dot{x}_{01}-v_{*}\right) \\
0 \\
-T\left(-\dot{x}_{02}-v_{*}\right)+\delta_{2}\left(x_{20}, \dot{x}_{20}\right)
\end{array}\right]
$$

Substituting the first column of the fundamental solution matrix (2.42) by vector $h\left(q_{02}, t\right)$, one obtains a matrix whose determinant is given below:

$K_{1-}\left(t, t_{0}\right)$

$$
=\left|\begin{array}{cccc}
0 & Q(t) \dot{r}(t) & P(-t) \exp (-\sqrt{1-2 c} t) & \dot{r}(t) \\
\gamma \cos \omega\left(t+t_{0}\right)+\delta_{1}\left(x_{10}, \dot{x}_{10}\right)-T\left(-\dot{x}_{01}-v_{*}\right) & (Q(t) \dot{r}(t))^{\bullet} & (P(-t) \exp (-\sqrt{1-2 c} t))^{\bullet} & \ddot{r}(t) \\
0 & Q(t) \dot{r}(t) & -P(-t) \exp (-\sqrt{1-2 c} t) & \dot{r}(t) \\
-T\left(-\dot{x}_{02}-v_{*}\right)+\delta_{2}\left(x_{20}, \dot{x}_{20}\right) & (Q(t) \dot{r}(t))^{\bullet} & -(P(-t) \exp (-\sqrt{1-2 c} t))^{\bullet} & \ddot{r}(t)
\end{array}\right| .
$$


16 Stick-slip chaos in a self-excited oscillator

Development with respect to the first column of the determinant yields

$$
\begin{aligned}
K_{1-}\left(t, t_{0}\right)= & 2 P(-t) \exp (-\sqrt{1-2 c} t) \\
\times\left(\gamma \cos \omega\left(t+t_{0}\right)+\delta_{1}\left(x_{10}, \dot{x}_{10}\right)-\delta_{2}\left(x_{20}, \dot{x}_{20}\right)\right. & \left.\quad-T\left(-\dot{x}_{01}-v_{*}\right)+T\left(-\dot{x}_{02}-v_{*}\right)\right) .
\end{aligned}
$$

In our case $x_{01}=x_{02}$ and therefore

$$
K_{1-}\left(t, t_{0}\right)=2 P(-t) \exp (-\sqrt{1-2 c} t)\left(\gamma \cos \omega\left(t+t_{0}\right)+\delta_{1}\left(x_{10}, \dot{x}_{10}\right)-\delta_{2}\left(x_{10}, \dot{x}_{10}\right)\right) .
$$

Substitution of the second column of the solution matrix $(2.42)$ by vector $h\left(q_{02}, t\right)$ gives the following determinant:

$K_{2-}\left(t, t_{0}\right)$

$$
=\left|\begin{array}{cccc}
P(t) \exp (D) & 0 & P(-t) \exp (-D) & \dot{r}(t) \\
(P(t) \exp (D))^{\bullet} & \gamma \cos \omega\left(t+t_{0}\right)+\delta_{1}\left(x_{10}, \dot{x}_{10}\right)-T\left(-\dot{x}_{01}-v_{*}\right) & (P(-t) \exp (-D))^{\bullet} & \ddot{r}(t) \\
-P(t) \exp (D) & 0 & -P(-t) \exp (-D) & \dot{r}(t) \\
-(P(t) \exp (D))^{\bullet} & -T\left(-\dot{x}_{02}-v_{*}\right)+\delta_{2}\left(x_{20}, \dot{x}_{20}\right) & -(P(-t) \exp (-D))^{\bullet} & \ddot{r}(t)
\end{array}\right|,
$$

Development with respect to the second column gives

$$
\begin{aligned}
K_{2-}\left(t, t_{0}\right)=2 A & \left(\gamma \cos \omega\left(t+t_{0}\right)+\delta_{1}\left(x_{10}, \dot{x}_{10}\right)\right. \\
+ & \left.\delta_{2}\left(x_{20}, \dot{x}_{20}\right)-T\left(-\dot{x}_{01}-v_{*}\right)-T\left(-\dot{x}_{02}-v_{*}\right)\right) \dot{r}(t),
\end{aligned}
$$

where

$$
A=\left|\begin{array}{cc}
P(t) \exp (\sqrt{1+2 a} t) & P(-t) \exp (-\sqrt{1-2 a} t) \\
(P(t) \exp (\sqrt{1+2 a} t))^{\bullet} & (P(-t) \exp (-\sqrt{1-2 a} t))^{\bullet}
\end{array}\right|=\text { const. }
$$

Since $x_{01}=x_{02}$, therefore

$$
K_{2-}\left(t, t_{0}\right)=2 A\left(\gamma \cos \omega\left(t+t_{0}\right)+\delta_{1}\left(x_{10}, \dot{x}_{10}\right)+\delta_{2}\left(x_{10}, \dot{x}_{10}\right)-2 T\left(-\dot{x}_{01}-v_{*}\right)\right) \dot{r}(t) .
$$


Substituting the third column of the fundamental solution matrix (2.42) by vector $h\left(q_{02}, t\right)$ gives the following determinant:

$K_{3-}\left(t, t_{0}\right)$

$$
=\left|\begin{array}{cccc}
P(t) \exp (\sqrt{1-2 c} t) & Q(t) \dot{r}(t) & 0 & \dot{r}(t) \\
(P(t) \exp (\sqrt{1-2 c} t))^{\bullet} & (Q(t) \dot{r}(t))^{\bullet} & \gamma \cos \omega\left(t+t_{0}\right)+\delta_{1}\left(x_{10}, \dot{x}_{10}\right)-T\left(-\dot{x}_{01}-v_{*}\right) & \ddot{r}(t) \\
-P(t) \exp (\sqrt{1-2 c} t) & Q(t) \dot{r}(t) & 0 & \dot{r}(t) \\
-(P(t) \exp (\sqrt{1-2 c} t))^{\bullet} & (Q(t) \dot{r}(t))^{\bullet} & -T\left(-\dot{x}_{02}-v_{*}\right)+\delta_{2}\left(x_{20}, \dot{x}_{20}\right) & \ddot{r}(t)
\end{array}\right| .
$$

Similarly, development with respect to the third column yields

$$
\begin{aligned}
K_{3-}\left(t, t_{0}\right)= & -2 P(t) \exp (\sqrt{1-2 c} t) \\
& \times\left(\gamma \cos \omega\left(t+t_{0}\right)+\delta_{1}\left(x_{10}, \dot{x}_{10}\right)+\delta_{2}\left(x_{20}, \dot{x}_{20}\right)-T\left(-\dot{x}_{01}-v_{*}\right)-T\left(-\dot{x}_{02}-v_{*}\right)\right),
\end{aligned}
$$

and for $x_{01}=x_{02}$ we have

$$
\begin{aligned}
K_{3-}\left(t, t_{0}\right)= & -2 P(t) \exp (\sqrt{1-2 c} t) \\
& \times\left(\gamma \cos \omega\left(t+t_{0}\right)+\delta_{1}\left(x_{10}, \dot{x}_{10}\right)+\delta_{2}\left(x_{10}, \dot{x}_{10}\right)-2 T\left(-\dot{x}_{01}-v_{*}\right)\right) .
\end{aligned}
$$

When substituting the fourth column of the fundamental solution matrix (2.42) by vector $h\left(q_{02}, t\right)$, one gets a matrix with the following determinant:

$K_{4-}\left(t, t_{0}\right)$

$=\left|\begin{array}{cccc}P(t) \exp (D) & Q(t) \dot{r}(t) & P(-t) \exp (-D) & 0 \\ (P(t) \exp (D))^{\bullet} & (Q(t) \dot{r}(t))^{\bullet} & (P(-t) \exp (-D))^{\bullet} & \gamma \cos \omega\left(t+t_{0}\right)+\delta_{1}\left(x_{10}, \dot{x}_{10}\right)-T\left(-\dot{x}_{01}-v_{*}\right) \\ -P(t) \exp (D) & Q(t) \dot{r}(t) & -P(-t) \exp (-D) t) & 0 \\ -(P(t) \exp (D))^{\bullet} & (Q(t) \dot{r}(t))^{\bullet} & -(P(-t) \exp (-D))^{\bullet} & -T\left(-\dot{x}_{02}-v_{*}\right)+\delta_{2}\left(x_{20}, \dot{x}_{20}\right)\end{array}\right|$,

where $D=\sqrt{1-2 c t}$.

Development with respect to the fourth column gives $\left|\lim _{t \rightarrow \pm \infty} K_{4-}\left(t, t_{0}\right)\right|=\infty$.

Perturbation vector along homoclinic orbits (2.19) for sign “+” is as follows:

$$
h\left(q_{03}, t\right)=\left[\begin{array}{c}
0 \\
\gamma \cos \omega\left(t+t_{0}\right)-\delta_{1}\left(x_{10}, \dot{x}_{10}\right)-T\left(\dot{x}_{01}-v_{*}\right) \\
0 \\
-T\left(\dot{x}_{02}-v_{*}\right)-\delta_{2}\left(x_{20}, \dot{x}_{20}\right)
\end{array}\right] .
$$


18 Stick-slip chaos in a self-excited oscillator

Substitution of the first column of the fundamental solution matrix (2.53) by vector $h\left(q_{03}, t\right)$ gives the following determinant:

$$
\begin{aligned}
& L_{1}\left(t, t_{0}\right) \\
& =\left|\begin{array}{cccc}
0 & Q(D) \dot{r}(D) & e^{-t} R(-D) & \dot{r}(D) \\
\gamma \cos \omega\left(t+t_{0}\right)-\delta_{1}\left(x_{10}, \dot{x}_{10}\right)-T\left(\dot{x}_{01}-v_{*}\right) & (Q(D) \dot{r}(D))^{\bullet} & \left(e^{-t} R(-D)\right)^{\bullet} & \ddot{r}(D) \\
0 & Q(D) \dot{r}(D) & -e^{-t} R(-D) & \dot{r}(D) \\
-T\left(\dot{x}_{02}-v_{*}\right)-\delta_{2}\left(x_{20}, \dot{x}_{20}\right) & (Q(D) \dot{r}(D))^{\bullet} & -\left(e^{-t} R(-D)\right)^{\bullet} & \ddot{r}(D)
\end{array}\right|, \\
& \text { where } D=\sqrt{1-2 c} t \text {. }
\end{aligned}
$$

Development with respect to the first column gives

$$
\begin{aligned}
L_{1}\left(t, t_{0}\right)= & 2 e^{-t} R(-\sqrt{1-2 c} t) \\
& \times\left(\gamma \cos \omega\left(t+t_{0}\right)-\delta_{1}\left(x_{10}, \dot{x}_{10}\right)+\delta_{2}\left(x_{20}, \dot{x}_{20}\right)-T\left(\dot{x}_{01}-v_{*}\right)+T\left(\dot{x}_{02}-v_{*}\right)\right),
\end{aligned}
$$

and because $x_{01}=-x_{02}$, one gets

$$
\begin{aligned}
L_{1}\left(t, t_{0}\right)= & 2 e^{-t} R(-\sqrt{1-2 c} t) \\
& \times\left(\gamma \cos \omega\left(t+t_{0}\right)-\delta_{1}\left(x_{10}, \dot{x}_{10}\right)+\delta_{2}\left(x_{10}, \dot{x}_{10}\right)-T\left(\dot{x}_{01}-v_{*}\right)+T\left(-\dot{x}_{01}-v_{*}\right)\right) .
\end{aligned}
$$

Substitution of the second column of the fundamental solution matrix (2.53) by the vector $h\left(q_{03}, t\right)$ gives a matrix and the associated determinant of the form

$$
\begin{aligned}
& L_{2}\left(t, t_{0}\right) \\
& =\left|\begin{array}{ccrc}
e^{t} R(D) & 0 & e^{-t} R(-D) & \dot{r}(D) \\
\left(e^{t} R(D)\right)^{\bullet} & \gamma \cos \omega\left(t+t_{0}\right)-\delta_{1}\left(x_{10}, \dot{x}_{10}\right)-T\left(\dot{x}_{01}-v_{*}\right) & \left(e^{-t} R(-D)\right) & \ddot{r}(D) \\
-e^{t} R(D) & 0 & -e^{-t} R(-D) & \dot{r}(D) \\
-\left(e^{t} R(D)\right)^{\bullet} & -T\left(\dot{x}_{02}-v_{*}\right)-\delta_{2}\left(x_{20}, \dot{x}_{20}\right) & -\left(e^{-t} R(-D)\right)^{\bullet} & \ddot{r}(D)
\end{array}\right|, \\
&
\end{aligned}
$$

Development with respect to the second column gives

$$
\begin{aligned}
L_{2}\left(t, t_{0}\right)=2 A & \left(\gamma \cos \omega\left(t+t_{0}\right)-\delta_{1}\left(x_{10}, \dot{x}_{10}\right)\right. \\
& \left.-\delta_{2}\left(x_{20}, \dot{x}_{20}\right)-T\left(\dot{x}_{01}-v_{*}\right)-T\left(\dot{x}_{02}-v_{*}\right)\right) \dot{r}(\sqrt{1-2 c} t) .
\end{aligned}
$$


Since $x_{01}=-x_{02}$, one gets

$$
\begin{aligned}
L_{2}\left(t, t_{0}\right)= & 2 A \dot{r}(\sqrt{1-2 c} t) \\
& \times\left(\gamma \cos \omega\left(t+t_{0}\right)-\delta_{1}\left(x_{10}, \dot{x}_{10}\right)+\delta_{2}\left(x_{10}, \dot{x}_{10}\right)-T\left(\dot{x}_{01}-v_{*}\right)-T\left(-\dot{x}_{01}-v_{*}\right)\right) .
\end{aligned}
$$

Substitution of the third column of the fundamental solution matrix (2.53) by vector $h\left(q_{03}, t\right)$ gives the following determinant:

$$
\begin{aligned}
& L_{3}\left(t, t_{0}\right) \\
& =\left|\begin{array}{cccc}
e^{t} R(D) & Q(D) \dot{r}(D) & 0 & \dot{r}(D) \\
\left(e^{t} R(D)\right)^{\bullet} & (Q(D) \dot{r}(D))^{\bullet} & \gamma \cos \omega\left(t+t_{0}\right)-\delta_{1}\left(x_{10}, \dot{x}_{10}\right)-T\left(\dot{x}_{01}-v_{*}\right) & \ddot{r}(D) \\
-e^{t} R(D) & Q(D) \dot{r}(D) & 0 & \dot{r}(D) \\
-\left(e^{t} R(D)\right)^{\bullet} & (Q(D) \dot{r}(D))^{\bullet} & -T\left(\dot{x}_{02}-v_{*}\right)-\delta_{2}\left(x_{20}, \dot{x}_{20}\right) & \ddot{r}(D)
\end{array}\right|,
\end{aligned}
$$

Development with respect to the third column gives

$$
\begin{aligned}
& L_{3}\left(t, t_{0}\right)=-2( \gamma \cos \omega\left(t+t_{0}\right)-\delta_{1}\left(x_{10}, \dot{x}_{10}\right)-\delta_{2}\left(x_{20}, \dot{x}_{20}\right) \\
&\left.-T\left(\dot{x}_{01}-v_{*}\right)-T\left(\dot{x}_{02}-v_{*}\right)\right) e^{t} R(\sqrt{1-2 c} t) .
\end{aligned}
$$

Since $x_{01}=-x_{02}$, one gets

$$
\begin{aligned}
L_{3}\left(t, t_{0}\right)= & -2 e^{t} R(\sqrt{1-2 c} t) \\
& \times\left(\gamma \cos \omega\left(t+t_{0}\right)-\delta_{1}\left(x_{10}, \dot{x}_{10}\right)+\delta_{2}\left(x_{10}, \dot{x}_{10}\right)-T\left(\dot{x}_{01}-v_{*}\right)-T\left(-\dot{x}_{01}-v_{*}\right)\right) .
\end{aligned}
$$

Substituting the fourth column of the fundamental solution matrix (2.53) by vector $h\left(q_{03}, t\right)$, a matrix with the following determinant is obtained:

$$
\begin{aligned}
& L_{4}\left(t, t_{0}\right) \\
& =\left|\begin{array}{cccc}
e^{t} R(D) & Q(D) \dot{r}(D) & e^{-t} R(-D) & 0 \\
\left(e^{t} R(D)\right)^{\bullet} & (Q(D) \dot{r}(D))^{\bullet} & \left(e^{-t} R(-D)\right)^{\bullet} & \gamma \cos \omega\left(t+t_{0}\right)-\delta_{1}\left(x_{10}, \dot{x}_{10}\right)-T\left(\dot{x}_{01}-v_{*}\right) \\
-e^{t} R(D) & Q(D) \dot{r}(D) & -e^{-t} R(-D) & 0 \\
-\left(e^{t} R(D)\right)^{\bullet} & (Q(D) \dot{r}(D))^{\bullet} & -\left(e^{-t} R(-D)\right)^{\bullet} & -T\left(\dot{x}_{02}-v_{*}\right)-\delta_{2}\left(x_{20}, \dot{x}_{20}\right)
\end{array}\right|,
\end{aligned}
$$

Development with respect to the third column gives $\left|\lim _{t \rightarrow \pm \infty} L_{4}\left(t, t_{0}\right)\right|=\infty$. 
The perturbation vector along homoclinic orbits (2.19) for interior sign is as follows:

$$
h\left(q_{04}, t\right)=\left[\begin{array}{c}
0 \\
\gamma \cos \omega\left(t+t_{0}\right)+\delta_{1}\left(x_{10}, \dot{x}_{10}\right)-T\left(-\dot{x}_{01}-v_{*}\right) \\
0 \\
-T\left(-\dot{x}_{02}-v_{*}\right)+\delta_{2}\left(x_{20}, \dot{x}_{20}\right)
\end{array}\right] .
$$

Substituting the first column of the fundamental solution matrix (2.53) by vector $h\left(q_{04}, t\right)$ gives the following determinant:

$$
\begin{aligned}
& L_{1-}\left(t, t_{0}\right) \\
& =\left|\begin{array}{cccc}
\gamma \cos \omega\left(t+t_{0}\right)+\delta_{1}\left(x_{10}, \dot{x}_{10}\right)-T\left(-\dot{x}_{01}-v_{*}\right) & (Q(D) \dot{r}(D))^{\bullet} & \left(e^{-t} R(-D)\right)^{\bullet} & \ddot{r}(D) \\
0 & Q(D) \dot{r}(D) & -e^{-t} R(-D) & \dot{r}(D) \\
-T\left(-\dot{x}_{02}-v_{*}\right)+\delta_{2}\left(x_{20}, \dot{x}_{20}\right) & (Q(D) \dot{r}(D))^{\bullet} & -\left(e^{-t} R(-D)\right)^{\bullet} & \ddot{r}(D)
\end{array}\right|, \\
& \\
& \text { where } D=\sqrt{1-2 c t} .
\end{aligned}
$$

Owing to the first column development, we get

$$
\begin{aligned}
L_{1-}\left(t, t_{0}\right)= & 2 e^{-t} R(-\sqrt{1-2 c} t) \\
& \times\left(\gamma \cos \omega\left(t+t_{0}\right)+\delta_{1}\left(x_{10}, \dot{x}_{10}\right)-\delta_{2}\left(x_{20}, \dot{x}_{20}\right)-T\left(-\dot{x}_{01}-v_{*}\right)+T\left(-\dot{x}_{02}-v_{*}\right)\right),
\end{aligned}
$$

and because $x_{01}=-x_{02}$, we have

$$
\begin{aligned}
L_{1-}\left(t, t_{0}\right)= & 2 e^{-t} R(-\sqrt{1-2 c} t) \\
& \times\left(\gamma \cos \omega\left(t+t_{0}\right)+\delta_{1}\left(x_{10}, \dot{x}_{10}\right)+\delta_{2}\left(x_{10}, \dot{x}_{10}\right)-T\left(-\dot{x}_{01}-v_{*}\right)+T\left(\dot{x}_{01}-v_{*}\right)\right) .
\end{aligned}
$$

Substituting the second column of $(2.53)$ by $h\left(q_{04}, t\right)$, we obtain

$$
\begin{aligned}
& L_{2-}\left(t, t_{0}\right) \\
& =\mid \begin{array}{cc}
e^{t} R(D) & 0 \\
\left(e^{t} R(D)\right)^{\bullet} & \gamma \cos \omega\left(t+t_{0}\right)+\delta_{1}\left(x_{10}, \dot{x}_{10}\right)-T\left(-\dot{x}_{01}-v_{*}\right) \\
-e^{t} R(D) & 0 \\
-\left(e^{t} R(D)\right)^{\bullet} & -T\left(-\dot{x}_{02}-v_{*}\right)+\delta_{2}\left(x_{20}, \dot{x}_{20}\right)
\end{array}
\end{aligned}
$$$$
\begin{array}{cc}
e^{-t} R(-D) & \dot{r}(D) \\
\left(e^{-t} R(-D)\right)^{\bullet} & \ddot{r}(D) \\
-e^{-t} R(-D) & \dot{r}(D) \\
-\left(e^{-t} R(-D)\right)^{\bullet} & \ddot{r}(D)
\end{array} \mid
$$$$
\text { where } D=\sqrt{1-2 c} t \text {. }
$$ 
Development with respect to the second column gives

$$
\begin{aligned}
L_{2-}\left(t, t_{0}\right)= & 2 A \dot{r}(\sqrt{1-2 c} t) \\
& \times\left(\gamma \cos \omega\left(t+t_{0}\right)+\delta_{1}\left(x_{10}, \dot{x}_{10}\right)+\delta_{2}\left(x_{20}, \dot{x}_{20}\right)-T\left(-\dot{x}_{01}-v_{*}\right)-T\left(-\dot{x}_{02}-v_{*}\right)\right) .
\end{aligned}
$$

Since $x_{01}=-x_{02}$, one gets

$$
\begin{aligned}
L_{2-}\left(t, t_{0}\right)= & 2 A \dot{r}(\sqrt{1-2 c} t) \\
& \times\left(\gamma \cos \omega\left(t+t_{0}\right)+\delta_{1}\left(x_{10}, \dot{x}_{10}\right)-\delta_{2}\left(x_{10}, \dot{x}_{10}\right)-T\left(-\dot{x}_{01}-v_{*}\right)-T\left(\dot{x}_{01}-v_{*}\right)\right) .
\end{aligned}
$$

Substituting the third column of the fundamental solution matrix (2.53) by vector $h\left(q_{04}, t\right)$ yields a matrix and then the determinant

$$
\begin{aligned}
& L_{3-}\left(t, t_{0}\right) \\
& =\left|\begin{array}{cccc}
e^{t} R(D) & Q(D) \dot{r}(D) & 0 & \dot{r}(D) \\
\left(e^{t} R(D)\right)^{\bullet} & (Q(D) \dot{r}(D))^{\bullet} & \gamma \cos \omega\left(t+t_{0}\right)+\delta_{1}\left(x_{10}, \dot{x}_{10}\right)-T\left(-\dot{x}_{01}-v_{*}\right) & \ddot{r}(D) \\
-e^{t} R(D) & Q(D) \dot{r}(D) & 0 & \dot{r}(D) \\
-\left(e^{t} R(D)\right)^{\bullet} & (Q(D) \dot{r}(D))^{\bullet} & -T\left(-\dot{x}_{02}-v_{*}\right)+\delta_{2}\left(x_{20}, \dot{x}_{20}\right) & \ddot{r}(D)
\end{array}\right|,
\end{aligned}
$$

A similar operation with respect to the third column gives

$$
\begin{aligned}
L_{3-}\left(t, t_{0}\right)= & -2 e^{t} R(\sqrt{1-2 c} t) \\
& \times\left(\gamma \cos \omega\left(t+t_{0}\right)+\delta_{1}\left(x_{10}, \dot{x}_{10}\right)+\delta_{2}\left(x_{20}, \dot{x}_{20}\right)-T\left(-\dot{x}_{01}-v_{*}\right)-T\left(-\dot{x}_{02}-v_{*}\right)\right)
\end{aligned}
$$

and owing to $x_{01}=-x_{02}$, one gets

$$
\begin{aligned}
L_{3-}\left(t, t_{0}\right)= & -2 e^{t} R(\sqrt{1-2 c} t) \\
& \times\left(\gamma \cos \omega\left(t+t_{0}\right)+\delta_{1}\left(x_{10}, \dot{x}_{10}\right)-\delta_{2}\left(x_{10}, \dot{x}_{10}\right)-T\left(-\dot{x}_{01}-v_{*}\right)-T\left(\dot{x}_{01}-v_{*}\right)\right) .
\end{aligned}
$$

Application of the described algorithm to column four gives

$L_{4-}\left(t, t_{0}\right)$

$$
=\left|\begin{array}{cccc}
e^{t} R(D) & Q(D) \dot{r}(D) & e^{-t} R(-D) & 0 \\
\left(e^{t} R(D)\right)^{\bullet} & (Q(D) \dot{r}(D))^{\bullet} & \left(e^{-t} R(-D)\right)^{\bullet} & \gamma \cos \omega\left(t+t_{0}\right)+\delta_{1}\left(x_{10}, \dot{x}_{10}\right)-T\left(-\dot{x}_{01}-v_{*}\right) \\
-e^{t} R(D) & Q(D) \dot{r}(D) & -e^{-t} R(-D) & 0 \\
-\left(e^{t} R(D)\right)^{\bullet} & (Q(D) \dot{r}(D))^{\bullet} & -\left(e^{-t} R(-D)\right)^{\bullet} & -T\left(-\dot{x}_{02}-v_{*}\right)+\delta_{2}\left(x_{20}, \dot{x}_{20}\right)
\end{array}\right|,
$$

and $\left|\lim _{t \rightarrow \pm \infty} L_{4-}\left(t, t_{0}\right)\right|=\infty$. 
22 Stick-slip chaos in a self-excited oscillator

According to (2.57), the Melnikov function has the following form:

$$
M_{1}\left(t_{0}\right)=M_{11}\left(t_{0}\right)+M_{12}
$$

where

$$
\begin{aligned}
M_{11}\left(t_{0}\right)= & 2 \gamma \int_{0}^{\infty} P(-t) \exp (-\sqrt{1-2 c} t) \cos \omega\left(t+t_{0}\right) d t \\
= & 2 \gamma \int_{0}^{\infty}\left(-\frac{2}{3} c+(\sqrt{1-2 c}+\operatorname{tgh} t) \operatorname{tgh} t\right) \exp (-\sqrt{1-2 c} t) \cos \omega\left(t+t_{0}\right) d t \\
M_{12}= & 2 \int_{0}^{\infty}\left(-\delta_{1}\left(x_{10}(t), \dot{x}_{10}(t)\right)+\delta_{2}\left(x_{10}(t), \dot{x}_{10}(t)\right)\right) P(-t) \exp (-\sqrt{1-2 c} t) d t \\
= & 2 \int_{0}^{\infty}\left(-\delta_{1}\left(x_{10}(t), \dot{x}_{10}(t)\right)+\delta_{2}\left(x_{10}(t), \dot{x}_{10}(t)\right)\right) \\
& \times\left(-\frac{2}{3} c+(\sqrt{1-2 c}+\operatorname{tgh} t) \operatorname{tgh} t\right) \exp (-\sqrt{1-2 c} t) d t
\end{aligned}
$$

On the other hand,

$$
M_{11}\left(t_{0}\right)=M_{111}\left(t_{0}\right)-M_{112}\left(t_{0}\right),
$$

where

$$
\begin{aligned}
& M_{111}\left(t_{0}\right)=2 \gamma \cos \omega t_{0} \int_{0}^{\infty}\left(-\frac{2}{3} c+(\sqrt{1-2 c}+\operatorname{tgh} t) \operatorname{tgh} t\right) \exp (-\sqrt{1-2 c} t) \cos \omega t d t \\
& M_{112}\left(t_{0}\right)=2 \gamma \sin \omega t_{0} \int_{0}^{\infty}\left(-\frac{2}{3} c+(\sqrt{1-2 c}+\operatorname{tgh} t) \operatorname{tgh} t\right) \exp (-\sqrt{1-2 c} t) \sin \omega t d t .
\end{aligned}
$$

Upon integration we get

$$
\begin{aligned}
M_{111}\left(t_{0}\right)=\frac{1}{6} \gamma \cos \left(\omega t_{0}\right)( & \frac{12 \sqrt{1-2 c}}{1-2 c+\omega^{2}}+\frac{12 \omega^{2}}{1-2 c+\omega^{2}}+\frac{8 a \sqrt{1-2 c}}{1-2 c+\omega^{2}} \\
& -3 \omega i \cdot \psi\left(\frac{1}{4}(\sqrt{1-2 c}-\omega i)\right)+3 \omega i \cdot \psi\left(\frac{1}{4}(\sqrt{1-2 c}+\omega i)\right) \\
+ & \left.3 \omega i \cdot \psi\left(\frac{1}{4}(2+\sqrt{1-2 c}-\omega i)\right)-3 \omega i \cdot \psi\left(\frac{1}{4}(2+\sqrt{1-2 c}+\omega i)\right)\right), \\
M_{312}\left(t_{0}\right)=\frac{1}{6} \gamma \omega \sin \left(\omega t_{0}\right)( & \frac{12 \sqrt{1-2 c}}{1-2 c+\omega^{2}}-\frac{12}{1-2 c+\omega^{2}}-\frac{8 a}{1-2 c+\omega^{2}} \\
& +3 \cdot \psi\left(\frac{1}{4}(\sqrt{1-2 c}-\omega i)\right)+3 \cdot \psi\left(\frac{1}{4}(\sqrt{1-2 c}+\omega i)\right) \\
& \left.-3 \cdot \psi\left(\frac{1}{4}(2+\sqrt{1-2 c}-\omega i)\right)-3 \cdot \psi\left(\frac{1}{4}(2+\sqrt{1-2 c}+\omega i)\right)\right),
\end{aligned}
$$


where $\psi(z)$ denotes the "digamma" function. It is defined as $\psi(z)=\Gamma^{\prime}(z) / \Gamma(z)$, where $\Gamma(z)$ is the Euler function. Since $\psi(\bar{z})=\overline{\psi(z)}$, one gets

$$
\begin{aligned}
M_{111}\left(t_{0}\right)=\gamma \cos \left(\omega t_{0}\right)( & \frac{(6-4 c) \sqrt{1-2 c}+6 \omega^{2}}{3\left(1-2 c+\omega^{2}\right)} \\
& \left.-\omega\left(\operatorname{Im} \psi\left(\frac{1}{4}(\sqrt{1-2 c}+\omega i)\right)-\operatorname{Im} \psi\left(\frac{1}{4}(2+\sqrt{1-2 c}+\omega i)\right)\right)\right), \\
M_{112}\left(t_{0}\right)=\gamma \sin \left(\omega t_{0}\right)( & \frac{6(\sqrt{1-2 c}-1)-4 c}{3\left(1-2 c+\omega^{2}\right)} \\
& \left.+\operatorname{Re} \psi\left(\frac{1}{4}(\sqrt{1-2 c}+\omega i)\right)-\operatorname{Re} \psi\left(\frac{1}{4}(2+\sqrt{1-2 c}+\omega i)\right)\right) .
\end{aligned}
$$

Substituting the above formulas into (2.102), we get

$$
M_{11}\left(t_{0}\right)=\gamma\left(A_{1} \cos \omega t_{0}-A_{2} \sin \omega t_{0}\right)=\tilde{A} \sin \left(\omega t_{0}-\kappa\right)
$$

where

$$
\begin{aligned}
& A_{1}=\frac{(6-4 c) \sqrt{1-2 c}+6 \omega^{2}}{3\left(1-2 c+\omega^{2}\right)}-\omega\left(\operatorname{Im} \psi\left(\frac{1}{4}(\sqrt{1-2 c}+\omega i)\right)-\operatorname{Im} \psi\left(\frac{1}{4}(2+\sqrt{1-2 c}+\omega i)\right)\right) \\
& A_{2}=\frac{5(\sqrt{1-2 c}-1)+4 c}{3\left(1-2 c+\omega^{2}\right)}+\operatorname{Re} \psi\left(\frac{1}{4}(\sqrt{1-2 c}+\omega i)\right)-\operatorname{Re} \psi\left(\frac{1}{4}(2+\sqrt{1-2 c}+\omega i)\right)
\end{aligned}
$$

and $\tilde{A}=\gamma \sqrt{A_{1}^{2}+A_{2}^{2}}, \operatorname{tg} \kappa=A_{1} / A_{2}$.

Expression $M_{12}\left(t_{0}\right)$ is defined as follows:

$$
M_{12}=M_{121}-M_{122}-M_{123}-M_{124},
$$

where

$$
\begin{aligned}
& M_{121}=2 \sqrt{2}\left(\alpha_{21}-\alpha_{11}\right) \int_{0}^{\infty} \operatorname{sech}(t)\left(-\frac{2}{3} c+(\sqrt{1-2 c}+\operatorname{tgh} t) \operatorname{tgh} t\right) \exp (-\sqrt{1-2 c} t) d t, \\
& M_{122}=2 \sqrt{2}\left(\delta_{21}-\delta_{11}\right) \int_{0}^{\infty} \operatorname{sech}(t) \operatorname{tgh}(t)\left(-\frac{2}{3} c+(\sqrt{1-2 c}+\operatorname{tgh} t) \operatorname{tgh} t\right) \exp (-\sqrt{1-2 c} t) d t, \\
& M_{123}=4\left(\delta_{22}-\delta_{12}\right) \int_{0}^{\infty} \operatorname{sech}^{2}(t) \operatorname{tgh}^{2}(t)\left(-\frac{2}{3} c+(\sqrt{1-2 c}+\operatorname{tgh} t) \operatorname{tgh} t\right) \exp (-\sqrt{1-2 c} t) d t, \\
& M_{124}=4 \sqrt{2}\left(\delta_{23}-\delta_{13}\right) \int_{0}^{\infty} \operatorname{sech}^{3}(t) \operatorname{tgh}^{3}(t)\left(-\frac{2}{3} c+(\sqrt{1-2 c}+\operatorname{tgh} t) \operatorname{tgh} t\right) \exp (-\sqrt{1-2 c} t) d t .
\end{aligned}
$$


Upon integration we have

$$
\begin{aligned}
& M_{121}=\frac{\sqrt{2}}{3}\left(\alpha_{21}-\alpha_{11}\right)\left(3 \sqrt{1-2 c}-c \psi\left(\frac{1}{4}(1+\sqrt{1-2 c})\right)+c \psi\left(\frac{1}{4}(3+\sqrt{1-2 c})\right)\right), \\
& M_{122}=-\frac{2 \sqrt{2}}{3}\left(\delta_{11}-\delta_{21}\right), \\
& M_{123}=-\frac{4\left(\delta_{22}-\delta_{12}\right)}{3(1+(1 / 2) \sqrt{1-2 c})(2+(1 / 2) \sqrt{1-2 c})(3+(1 / 2) \sqrt{1-2 c})} \\
& \times\left(-4(3-2 c+3 \sqrt{1-2 c}) F\left(6,1+\frac{1}{2} \sqrt{1-2 c} ; 4+\frac{1}{2} \sqrt{1-2 c},-1\right)+\frac{3+2 c}{4+(1 / 2) \sqrt{1-2 c}}\right. \\
& \left(4(2+\sqrt{1-2 c}) F\left(6,2+\frac{1}{2} \sqrt{1-2 c} ; 5+\frac{1}{2} \sqrt{1-2 c},-1\right)\right. \\
& \left.\left.+\frac{-3-2 a+3 \sqrt{1-2 c}}{5+(1 / 2) \sqrt{1-2 c}} F\left(6,3+\frac{1}{2} \sqrt{1-2 c} ; 6+\frac{1}{2} \sqrt{1-2 c},-1\right)\right)\right) \\
& M_{124}=-\frac{1024 \sqrt{2}\left(\delta_{23}-\delta_{13}\right)}{(3+\sqrt{1-2 c})(5+\sqrt{1-2 c})(7+\sqrt{1-2 c})(9+\sqrt{1-2 c})(11+\sqrt{1-2 c})(13+\sqrt{1-2 c})} \\
& \times\left(\left(3 a(73+9 \sqrt{1-2 c})-252(1+\sqrt{1-2 c})-2 c^{2}\right)\right. \\
& F\left(8, \frac{1}{2}(3+\sqrt{1-2 c}) ; \frac{1}{2}(11+\sqrt{1-2 c}) ;-1\right) \\
& +2(3+2 c)(20-c+8 \sqrt{1-2 c}) F\left(8, \frac{1}{2}(5+\sqrt{1-2 c}) ; \frac{1}{2}(13+\sqrt{1-2 c}) ;-1\right) \\
& \left.+\left((12+5 a)(-1+\sqrt{1-2 c})-2 a^{2}\right) F\left(8, \frac{1}{2}(7+\sqrt{1-2 c}) ; \frac{1}{2}(15+\sqrt{1-2 c}) ;-1\right)\right),
\end{aligned}
$$

where $F(a, b ; c ; d)$ denotes the hypergeometric function defined as

$$
F(a ; b ; c ; z)=\frac{\Gamma(c)}{\Gamma(b) \Gamma(c-b)} \int_{0}^{1} t^{b-1}(1-t)^{c-b-1}(1-2 t)^{-a} d t .
$$

One gets

$$
\begin{aligned}
M_{12}= & \frac{\sqrt{2}}{3}\left(\alpha_{21}-\alpha_{11}\right)\left(3 \sqrt{1-2 c}-c \psi\left(\frac{1}{4}(1+\sqrt{1-2 c})\right)+c \psi\left(\frac{1}{4}(3+\sqrt{1-2 c})\right)\right) \\
& +\frac{2 \sqrt{2}}{3}\left(\delta_{11}-\delta_{21}\right)-M_{123}-M_{124} .
\end{aligned}
$$


Substituting (2.106) and (2.112) into (2.100), we have

$$
\begin{aligned}
M_{1}\left(t_{0}\right)= & \tilde{A} \sin \left(\omega t_{0}-\kappa\right)+\frac{2 \sqrt{2}}{3}\left(\delta_{11}-\delta_{21}\right)-M_{123}-M_{124} \\
& +\frac{\sqrt{2}}{3}\left(\alpha_{21}-\alpha_{11}\right)\left(3 \sqrt{1-2 c}-c \psi\left(\frac{1}{4}(1+\sqrt{1-2 c})\right)+c \psi\left(\frac{1}{4}(3+\sqrt{1-2 c})\right)\right) .
\end{aligned}
$$

Finally, the following chaos criterion is obtained:

$$
\begin{aligned}
\tilde{A}>\mid & \frac{2 \sqrt{2}}{3}\left(\delta_{11}-\delta_{21}\right)-M_{123}-M_{124} \\
& +\frac{\sqrt{2}}{3}\left(\alpha_{21}-\alpha_{11}\right)\left(3 \sqrt{1-2 c}-c \psi\left(\frac{1}{4}(1+\sqrt{1-2 c})\right)+c \psi\left(\frac{1}{4}(3+\sqrt{1-2 c})\right)\right) \mid .
\end{aligned}
$$

According to (2.61), the Melnikov function is

$$
M_{2}\left(t_{0}\right)=M_{21}\left(t_{0}\right)-M_{22}-M_{23}
$$

where

$$
\begin{gathered}
M_{21}\left(t_{0}\right)=2 A \gamma \int_{-\infty}^{\infty} \dot{r}(t) \cos \omega\left(t+t_{0}\right) d t=-2 A \gamma \int_{-\infty}^{\infty} \operatorname{sech} t \operatorname{tgh} t \cos \omega\left(t+t_{0}\right) d t, \\
M_{22}=2 A \int_{-\infty}^{\infty}\left(\delta_{1}\left(x_{10}, \dot{x}_{10}\right)+\delta_{2}\left(x_{10}, \dot{x}_{10}\right)\right) \dot{r}(t) d t \\
M_{23}=4 A \int_{-\infty}^{\infty} T\left(\dot{x}_{01}-v_{*}\right) \dot{r}(t) d t=-4 A \int_{-\infty}^{\infty} T\left(\dot{x}_{01}-v_{*}\right) \operatorname{sech} t \operatorname{tgh} t d t .
\end{gathered}
$$

Formula $M_{21}\left(t_{0}\right)$ is as follows:

$$
M_{21}\left(t_{0}\right)=-M_{211}\left(t_{0}\right)+M_{212}\left(t_{0}\right),
$$

where

$$
\begin{gathered}
M_{211}=2 A \gamma \cos \omega t_{0} \int_{-\infty}^{\infty} \operatorname{sech} t \operatorname{tgh} t \cos \omega t d t=0 \\
M_{212}=2 A \gamma \sin \omega t_{0} \int_{-\infty}^{\infty} \operatorname{sech} t \operatorname{tgh} t \sin \omega t d t=2 \pi A \gamma \omega \operatorname{sech}\left(\frac{\pi \omega}{2}\right) \sin \omega t_{0}
\end{gathered}
$$

Substituting the obtained formulas into (2.117), one gets

$$
M_{21}\left(t_{0}\right)=M_{212}\left(t_{0}\right)=2 \pi A \gamma \omega \operatorname{sech}\left(\frac{\pi \omega}{2}\right) \sin \omega t_{0} .
$$

Integral $M_{22}$ is defined in the following way:

$$
M_{22}=-M_{221}+M_{222}+M_{223}+M_{224},
$$


26 Stick-slip chaos in a self-excited oscillator

where

$$
\begin{aligned}
& M_{221}=2 \sqrt{2} A\left(\alpha_{11}+\alpha_{21}\right) \int_{-\infty}^{\infty} \operatorname{sech}^{2}(t) \operatorname{tgh}(t) d t, \\
& M_{222}=2 \sqrt{2} A\left(\delta_{11}+\delta_{21}\right) \int_{-\infty}^{\infty} \operatorname{sech}^{2}(t) \operatorname{tgh}^{2}(t) d t, \\
& M_{223}=4 A\left(\delta_{12}+\delta_{22}\right) \int_{-\infty}^{\infty} \operatorname{sech}^{3}(t) \operatorname{tgh}^{3}(t) \operatorname{sgn}(\operatorname{sech}(t) \operatorname{tgh}(t)) d t, \\
& M_{224}=4 \sqrt{2} A\left(\delta_{13}+\delta_{23}\right) \int_{-\infty}^{\infty} \operatorname{sech}^{4}(t) \operatorname{tgh}^{4}(t) d t .
\end{aligned}
$$

Upon integration we get

$$
\begin{aligned}
M_{221} & =0, \\
M_{222} & =\left.\frac{2 \sqrt{2}}{3} A\left(\delta_{11}+\delta_{21}\right) \operatorname{tgh}^{3}(\sqrt{a} t)\right|_{-\infty} ^{\infty}=\frac{4 \sqrt{2}}{3} A\left(\delta_{11}+\delta_{21}\right), \\
M_{223} & =8 A\left(\delta_{12}+\delta_{22}\right) \int_{0}^{\infty} \operatorname{sech}^{3}(t) \operatorname{tgh}^{3}(t) d t \\
& =\left.8 A\left(\delta_{12}+\delta_{22}\right)\left(\frac{1}{5} \operatorname{sech}^{5}(t)-\frac{1}{3} \operatorname{sech}^{3}(t)\right)\right|_{0} ^{\infty}=\frac{16}{15} A\left(\delta_{12}+\delta_{22}\right), \\
M_{224} & =\frac{16}{35} \sqrt{2} A\left(\delta_{13}+\delta_{23}\right),
\end{aligned}
$$

and hence

$$
M_{22}=4 A\left(\frac{\sqrt{2}}{3}\left(\delta_{11}+\delta_{21}\right)+\frac{4}{15}\left(\delta_{12}+\delta_{22}\right)+\frac{4}{35} \sqrt{2}\left(\delta_{13}+\delta_{23}\right)\right) .
$$

Taking into account friction characteristics defined by (2.5), one gets

$$
M_{23}=-M_{231}-M_{232}+M_{233}+M_{234}+M_{235},
$$

where

$$
\begin{aligned}
& M_{231}=4 A T_{0} \int_{-\infty}^{\infty} \operatorname{sgn}\left(\dot{x}_{01}(t)-v_{*}\right) \operatorname{sech} t \operatorname{tgh} t d t, \\
& M_{232}=4 A\left(v_{*}-\beta v_{*}^{3}\right) \int_{-\infty}^{\infty} \operatorname{sech} t \operatorname{tgh} t d t d t, \\
& M_{233}=4 \sqrt{2} A\left(3 \beta v_{*}^{2}-\alpha\right) \int_{-\infty}^{\infty} \operatorname{sech}^{2} t \operatorname{tgh}^{2} t d t, \\
& M_{234}=12 \sqrt{2} A \beta v_{*} \int_{-\infty}^{\infty} \operatorname{sech}^{3} t \operatorname{tgh}^{3} t d t, \\
& M_{235}=4 \sqrt{2} A \beta \int_{-\infty}^{\infty} \operatorname{sech}^{4} t \operatorname{tgh}^{4} t d t .
\end{aligned}
$$


Note that the value of integral $M_{231}$ depends on the sign of $\Delta=1-2 v_{*}^{2}$. So, we consider first the case $\Delta<0$. In this case, $\operatorname{sgn}\left(\dot{x}_{01}(t)-v_{*}\right)=-1$ and

$$
M_{231}=-4 A T_{0} \int_{-\infty}^{\infty} \operatorname{sech} t \operatorname{tgh} t d t=0 .
$$

In the second case, that is, for $\Delta \geq 0$, one gets

$$
M_{231}=4 A T_{0}\left(-\int_{-\infty}^{t_{1}} \operatorname{sech} t \operatorname{tgh} t d t+\int_{t_{1}}^{t_{2}} \operatorname{sech} t \operatorname{tgh} t d t-\int_{t_{2}}^{\infty} \operatorname{sech} t \operatorname{tgh} t d t\right)
$$

where $t_{1}, t_{2}$ are defined by

$$
\begin{aligned}
& t_{1}=\frac{-1}{\sqrt{1-2 c}} \operatorname{arcsech} \sqrt{\frac{1}{2}+\sqrt{\frac{1}{4}-\frac{v_{*}^{2}}{2(1-2 c)^{2}}}}, \\
& t_{2}=\frac{-1}{\sqrt{1-2 c}} \operatorname{arcsech} \sqrt{\frac{1}{2}+\sqrt{\frac{1}{4}-\frac{v_{*}^{2}}{2(1-2 c)^{2}}}},
\end{aligned}
$$

Upon integration we have

$$
M_{231}=4 A T_{0}\left(\left.\operatorname{sech} t\right|_{-\infty} ^{t_{1}}-\left.\operatorname{sech} t\right|_{t_{1}} ^{t_{2}}+\left.\operatorname{sech} t\right|_{t_{2}} ^{\infty}\right)=8 A T_{0}\left(\operatorname{sech} t_{1}-\operatorname{sech} t_{2}\right)
$$

and one finds

$$
M_{231}=8 A T_{0}\left(\sqrt{\frac{1}{2}+\sqrt{\frac{1}{4}-\frac{v_{*}^{2}}{2}}}-\sqrt{\frac{1}{2}-\sqrt{\frac{1}{4}-\frac{v_{*}^{2}}{2}}}\right)
$$

The main result of our first part consideration is

$$
M_{231}= \begin{cases}8 A T_{0}\left(\sqrt{\frac{1}{2}+\sqrt{\frac{1}{4}-\frac{v_{*}^{2}}{2}}}-\sqrt{\left.\frac{1}{2}-\sqrt{\frac{1}{4}-\frac{v_{*}^{2}}{2}}\right)}\right. & \text { for } v_{*}<\frac{\sqrt{2}}{2} \\ 0 & \text { for } v_{*} \geq \frac{\sqrt{2}}{2}\end{cases}
$$

Observe that

$$
M_{232}=M_{234}=0
$$


28 Stick-slip chaos in a self-excited oscillator

and that

$$
M_{233}=\left.\frac{4 \sqrt{2}}{3} A\left(3 \beta v_{*}^{2}-\alpha\right) \operatorname{tgh}^{3} t\right|_{-\infty} ^{\infty}
$$

and hence one gets

$$
M_{233}=\frac{8 \sqrt{2}}{3} A\left(3 \beta v_{*}^{2}-\alpha\right) .
$$

Integral $M_{235}$ gives

$$
M_{235}=\left.\frac{4 \sqrt{2} A \beta}{35}(6+\cosh (2 t)) \operatorname{sech}^{2} t \operatorname{tgh}^{5} t\right|_{-\infty} ^{\infty},
$$

and therefore

$$
M_{235}=\frac{16 \sqrt{2} A \beta}{35} .
$$

Substituting (2.135), (2.136), (2.138), and (2.140) into (2.124), we have

$$
\begin{aligned}
M_{23}= & \frac{8 \sqrt{2}}{3} A\left(3 \beta v_{*}^{2}-\alpha\right)+\frac{16 \sqrt{2} A \beta}{35} \\
& - \begin{cases}8 A T_{0}\left(\sqrt{\frac{1}{2}+\sqrt{\frac{1}{4}-\frac{v_{*}^{2}}{2}}}-\sqrt{\frac{1}{2}-\sqrt{\frac{1}{4}-\frac{v_{*}^{2}}{2}}}\right) & \text { for } v_{*}<\frac{\sqrt{2}}{2}, \\
0 & \text { for } v_{*} \geq \frac{\sqrt{2}}{2} .\end{cases}
\end{aligned}
$$

Substitution of (2.119), (2.123), and (2.141) into (2.115) gives the following Melnikov function:

$$
\begin{aligned}
M_{2}\left(t_{0}\right)= & 2 \pi A \gamma \omega \operatorname{sech}\left(\frac{\pi \omega}{2}\right) \sin \omega t_{0} \\
& -4 A\left(\frac{\sqrt{2}}{3}\left(\delta_{11}+\delta_{21}\right)+\frac{4}{15}\left(\delta_{12}+\delta_{22}\right)+\frac{4}{35} \sqrt{2}\left(\delta_{13}+\delta_{23}\right)\right) \\
& -\frac{8 \sqrt{2}}{3} A\left(3 \beta v_{*}^{2}-\alpha\right)-\frac{16 \sqrt{2} A \beta}{35} \\
& + \begin{cases}8 A T_{0}\left(\sqrt{\frac{1}{2}+\sqrt{\frac{1}{4}-\frac{v_{*}^{2}}{2}}}-\sqrt{\frac{1}{2}-\sqrt{\frac{1}{4}-\frac{v_{*}^{2}}{2}}}\right) & \text { for } v_{*}<\frac{\sqrt{2}}{2}, \\
0 & \text { for } v_{*} \geq \frac{\sqrt{2}}{2} .\end{cases}
\end{aligned}
$$


Intersection condition of stable and unstable manifolds is as follows:

$$
\begin{aligned}
& 2 \pi \gamma \omega|A| \operatorname{sech}\left(\frac{\pi \omega}{2}\right) \\
& >-4 A\left(\frac{\sqrt{2}}{3}\left(\delta_{11}+\delta_{21}\right)+\frac{4}{15}\left(\delta_{12}+\delta_{22}\right)+\frac{4}{35} \sqrt{2}\left(\delta_{13}+\delta_{23}\right)\right) \\
& -\frac{8 \sqrt{2}}{3} A\left(3 \beta v_{*}^{2}-\alpha\right)-\frac{16 \sqrt{2} A \beta}{35} \\
& +\left\{\begin{array}{ll}
8 A T_{0}\left(\sqrt{\frac{1}{2}+\sqrt{\frac{1}{4}-\frac{v_{*}^{2}}{2}}}-\sqrt{\left.\frac{1}{2}-\sqrt{\frac{1}{4}-\frac{v_{*}^{2}}{2}}\right)}\right. & \text { for } v_{*}<\frac{\sqrt{2}}{2} \\
0 & \text { for } v_{*} \geq \frac{\sqrt{2}}{2}
\end{array} \mid .\right.
\end{aligned}
$$

According to (2.64) the Melnikov function is defined in the following way:

$$
M_{3}\left(t_{0}\right)=-M_{31}\left(t_{0}\right)+M_{32}+M_{33}
$$

where

$$
\begin{aligned}
M_{31}\left(t_{0}\right) & =2 \gamma \int_{-\infty}^{0} P(t) \exp (\sqrt{1-2 c} t) \cos \omega\left(t+t_{0}\right) d t \\
& =2 \gamma \int_{-\infty}^{0}\left(-\frac{2}{3} c-(\sqrt{1-2 c}-\operatorname{tgh} t) \operatorname{tgh} t\right) \exp (\sqrt{1-2 c} t) \cos \omega\left(t+t_{0}\right) d t \\
M_{32} & =2 \int_{-\infty}^{0}\left(\delta_{1}\left(x_{10}(t), \dot{x}_{10}(t)\right)+\delta_{2}\left(x_{10}(t), \dot{x}_{10}(t)\right)\right) P(t) \exp (\sqrt{1-2 c} t) d t \\
M_{33} & =4 \int_{-\infty}^{0} T\left(\dot{x}_{01}-v_{*}\right) P(t) \exp (\sqrt{1-2 c} t) d t \\
& =4 \int_{-\infty}^{0} T\left(\dot{x}_{01}-v_{*}\right)\left(-\frac{2}{3} c-(\sqrt{1-2 c}-\operatorname{tgh} t) \operatorname{tgh} t\right) \exp (\sqrt{1-2 c} t) d t
\end{aligned}
$$

Formula $M_{31}\left(t_{0}\right)$ is

$$
M_{31}\left(t_{0}\right)=M_{311}\left(t_{0}\right)-M_{312}\left(t_{0}\right),
$$


where

$$
\begin{aligned}
& M_{311}\left(t_{0}\right)=2 \gamma \cos \omega t_{0} \int_{-\infty}^{0}\left(-\frac{2}{3} c-(\sqrt{1-2 c}-\operatorname{tgh} t) \operatorname{tgh} t\right) \exp (\sqrt{1-2 c} t) \cos \omega t d t \\
& M_{312}\left(t_{0}\right)=2 \gamma \sin \omega t_{0} \int_{-\infty}^{0}\left(-\frac{2}{3} c-(\sqrt{1-2 c}-\operatorname{tgh} t) \operatorname{tgh} t\right) \exp (\sqrt{1-2 c} t) \sin \omega t d t
\end{aligned}
$$

Upon integration we have

$$
\begin{aligned}
M_{311}\left(t_{0}\right)=\frac{1}{6} \gamma \cos \left(\omega t_{0}\right)( & \frac{(12-8 c) \sqrt{1-2 c}+12 \omega^{2}}{1-2 c+\omega^{2}} \\
& -3 \omega i \cdot \psi\left(\frac{1}{4}(\sqrt{1-2 c}-\omega i)\right)+3 \omega i \cdot \psi\left(\frac{1}{4}(\sqrt{1-2 c}+\omega i)\right) \\
+ & \left.3 \omega i \cdot \psi\left(\frac{1}{4}(2+\sqrt{1-2 c}-\omega i)\right)-3 \omega i \cdot \psi\left(\frac{1}{4}(2+\sqrt{1-2 c}+\omega i)\right)\right), \\
M_{312}\left(t_{0}\right)=-\frac{1}{6} \gamma \cos \left(\omega t_{0}\right)( & \frac{12(\sqrt{1-2 c}-1)+8 c}{1-2 c+\omega^{2}} \\
& +3 \cdot \psi\left(\frac{1}{4}(\sqrt{1-2 c}+\omega i)\right)+3 \cdot \psi\left(\frac{1}{4}(\sqrt{1-2 c}-\omega i)\right) \\
& \left.-3 \cdot \psi\left(\frac{1}{4}(2+\sqrt{1-2 c}+\omega i)\right)-3 \cdot \psi\left(\frac{1}{4}(2+\sqrt{1-2 c}-\omega i)\right)\right) .
\end{aligned}
$$

Since $\psi(\bar{z})=\overline{\psi(z)}$, one gets

$$
\begin{aligned}
M_{311}\left(t_{0}\right)=\gamma \cos \left(\omega t_{0}\right)( & \frac{(6-4 c) \sqrt{1-2 c}+6 \omega^{2}}{3\left(1-2 c+\omega^{2}\right)} \\
& \left.-\omega\left(\operatorname{Im} \psi\left(\frac{1}{4}(\sqrt{1-2 c}+\omega i)\right)-\operatorname{Im} \psi\left(\frac{1}{4}(2+\sqrt{1-2 c}+\omega i)\right)\right)\right), \\
M_{312}\left(t_{0}\right)=-\gamma \sin \left(\omega t_{0}\right)( & \frac{6(\sqrt{1-2 c}-1)+4 c}{3\left(1-2 c+\omega^{2}\right)} \\
& \left.+\operatorname{Re} \psi\left(\frac{1}{4}(\sqrt{1-2 c}+\omega i)\right)-\operatorname{Re} \psi\left(\frac{1}{4}(2+\sqrt{1-2 c}+\omega i)\right)\right) .
\end{aligned}
$$

Observe that the following relations hold $M_{311}\left(t_{0}\right)=M_{111}\left(t_{0}\right), M_{312}\left(t_{0}\right)=-M_{112}\left(t_{0}\right)$. Substituting the obtained formulas into (2.146), one gets

$$
M_{31}\left(t_{0}\right)=\gamma\left(A_{1} \cos \omega t_{0}+A_{2} \sin \omega t_{0}\right)=\tilde{A} \sin \left(\omega t_{0}+\kappa\right),
$$


where

$$
\begin{aligned}
& A_{1}=\frac{(6-4 c) \sqrt{1-2 c}+6 \omega^{2}}{3\left(1-2 c+\omega^{2}\right)}-\omega\left(\operatorname{Im} \psi\left(\frac{1}{4}(\sqrt{1-2 c}+\omega i)\right)-\operatorname{Im} \psi\left(\frac{1}{4}(2+\sqrt{1-2 c}+\omega i)\right)\right), \\
& A_{2}=\frac{5(\sqrt{1-2 c}-1)+4 c}{3\left(1-2 c+\omega^{2}\right)}+\operatorname{Re} \psi\left(\frac{1}{4}(\sqrt{1-2 c}+\omega i)\right)-\operatorname{Re} \psi\left(\frac{1}{4}(2+\sqrt{1-2 c}+\omega i)\right),
\end{aligned}
$$

and $\tilde{A}=\gamma \sqrt{A_{1}^{2}+A_{2}^{2}}, \operatorname{tg} \kappa=A_{1} / A_{2}$.

Formula $M_{32}\left(t_{0}\right)$ is defined as follows:

$$
M_{32}=M_{321}-M_{322}-M_{323}-M_{324},
$$

where

$$
\begin{aligned}
& M_{321}=2 \sqrt{2}\left(\alpha_{11}+\alpha_{21}\right) \int_{-\infty}^{0} \operatorname{sech}(t) P(t) \exp (\sqrt{1-2 c} t) d t, \\
& M_{322}=2 \sqrt{2}\left(\delta_{11}+\delta_{21}\right) \int_{-\infty}^{0} \operatorname{sech}(t) \operatorname{tgh}(t) P(t) \exp (\sqrt{1-2 c} t) d t, \\
& M_{323}=4\left(\delta_{12}+\delta_{22}\right) \int_{-\infty}^{0} \operatorname{sech}^{2}(t) \operatorname{tgh}^{2}(t) \operatorname{sgn}(\operatorname{sech}(t) \operatorname{tgh}(t)) P(t) \exp (\sqrt{1-2 c} t) d t, \\
& M_{324}=4 \sqrt{2}\left(\delta_{13}+\delta_{23}\right) \int_{-\infty}^{0} \operatorname{sech}(t)^{3} \operatorname{tgh}^{3}(t) P(t) \exp (\sqrt{1-2 c} t) d t .
\end{aligned}
$$

\section{Computations give}

$$
\begin{aligned}
M_{321}= & \frac{\sqrt{2}}{3}\left(\alpha_{21}+\alpha_{11}\right)\left(3 \sqrt{1-2 c}-c \psi\left(\frac{1}{4}(1+\sqrt{1-2 c})\right)+c \psi\left(\frac{1}{4}(3+\sqrt{1-2 c})\right)\right) \\
M_{322}= & -\frac{2 \sqrt{2}}{3}\left(\delta_{11}+\delta_{21}\right) \\
M_{323}= & -\frac{128\left(\delta_{22}+\delta_{12}\right)}{3(2+\sqrt{1-2 c})(4+\sqrt{1-2 c})(6+\sqrt{1-2 c})(8+\sqrt{1-2 c})(10+\sqrt{1-2 c})} \\
& \times\left(\left(6 a(46+7 \sqrt{1-2 c})-297(1+\sqrt{1-2 c})-4 c^{2}\right)\right. \\
& \quad F\left(6, \frac{1}{2}(2+\sqrt{1-2 c}) ; \frac{1}{2}(8+\sqrt{1-2 c}) ;-1\right) \\
& \quad+(3+2 c)\left((42-4 c+24 \sqrt{1-2 c}) F\left(6, \frac{1}{2}(4+\sqrt{1-2 c}) ; \frac{1}{2}(10+\sqrt{1-2 c}) ;-1\right)\right. \\
& \left.\left.\quad+(3(-1+\sqrt{1-2 c})-2 a)+F\left(6, \frac{1}{2}(6+\sqrt{1-2 c}) ; \frac{1}{2}(12+\sqrt{1-2 c}) ;-1\right)\right)\right)
\end{aligned}
$$




$$
\begin{aligned}
M_{324}=- & \frac{1024 \sqrt{2}\left(\delta_{23}+\delta_{13}\right)}{(3+\sqrt{1-2 c})(5+\sqrt{1-2 c})(7+\sqrt{1-2 c})(9+\sqrt{1-2 c})(11+\sqrt{1-2 c})(13+\sqrt{1-2 c})} \\
\times & \left(\left(3 a(73+9 \sqrt{1-2 c})-252(1+\sqrt{1-2 c})-2 c^{2}\right)\right. \\
& F\left(8, \frac{1}{2}(3+\sqrt{1-2 c}) ; \frac{1}{2}(11+\sqrt{1-2 c}) ;-1\right) \\
& +2(3+2 c)(20-c+8 \sqrt{1-2 c}) F\left(8, \frac{1}{2}(5+\sqrt{1-2 c}) ; \frac{1}{2}(13+\sqrt{1-2 c}) ;-1\right) \\
& \left.+\left((12+5 a)(-1+\sqrt{1-2 c})-2 a^{2}\right) F\left(8, \frac{1}{2}(7+\sqrt{1-2 c}) ; \frac{1}{2}(15+\sqrt{1-2 c}) ;-1\right)\right),
\end{aligned}
$$

and hence

$$
\begin{aligned}
M_{32}= & \frac{\sqrt{2}}{3}\left(\alpha_{21}+\alpha_{11}\right)\left(3 \sqrt{1-2 c}-c \psi\left(\frac{1}{4}(1+\sqrt{1-2 c})\right)+c \psi\left(\frac{1}{4}(3+\sqrt{1-2 c})\right)\right) \\
& +\frac{2 \sqrt{2}}{3}\left(\delta_{11}+\delta_{21}\right)-M_{323}-M_{324} .
\end{aligned}
$$

According to (2.5), one gets

$$
M_{33}=M_{331}+M_{332}-M_{333}-M_{334}-M_{335},
$$

where the following notation is applied:

$$
\begin{aligned}
& M_{331}=4 T_{0} \int_{-\infty}^{0} \operatorname{sgn}\left(\dot{x}-v_{*}\right)\left(-\frac{2}{3} c-(\sqrt{1-2 c}-\operatorname{tgh} t) \operatorname{tgh} t\right) \exp (\sqrt{1-2 c} t) d t \\
& M_{332}=4 v_{*}\left(1-\beta v_{*}^{2}\right) \int_{-\infty}^{0}\left(-\frac{2}{3} c-(\sqrt{1-2 c}-\operatorname{tgh} t) \operatorname{tgh} t\right) \exp (\sqrt{1-2 c} t) d t \\
& M_{333}=4 \sqrt{2}\left(3 \beta v_{*}^{2}-\alpha\right) \int_{-\infty}^{0}\left(-\frac{2}{3} c-(\sqrt{1-2 c}-\operatorname{tgh} t) \operatorname{tgh} t\right) \exp (\sqrt{1-2 c} t) \operatorname{sech} t \operatorname{tgh} t d t \\
& M_{334}=24 \beta v_{*} \int_{-\infty}^{0}\left(-\frac{2}{3} c-(\sqrt{1-2 c}-\operatorname{tgh} t) \operatorname{tgh} t\right) \exp (\sqrt{1-2 c} t) \operatorname{sech}^{2} \operatorname{tgh}^{2} t d t \\
& M_{335}=8 \sqrt{2} \beta \int_{-\infty}^{0}\left(-\frac{2}{3} c-(\sqrt{1-2 c}-\operatorname{tgh} t) \operatorname{tgh} t\right) \exp (\sqrt{1-2 c} t) \operatorname{sech}^{3} t \operatorname{tgh}^{3} t d t
\end{aligned}
$$

For $v_{*}>\sqrt{2} / 2$, integral $M_{331}$ is defined as follows:

$$
M_{331}=-4 T_{0} \int_{-\infty}^{0}\left(-\frac{2}{3} c-(\sqrt{1-2 c}-\operatorname{tgh} t) \operatorname{tgh} t\right) \exp (\sqrt{1-2 c} t) d t .
$$


Looking for a primary function, we have

$$
\begin{aligned}
\widetilde{M}_{331} & =4 T_{0} \int\left(-\frac{2}{3} c-(\sqrt{1-2 c}-\operatorname{tgh} t) \operatorname{tgh} t\right) \exp (\sqrt{1-2 c} t) d t \\
& =\frac{4 T_{0} e^{\sqrt{1-2 c} t}(3-2 c-3 \sqrt{1-2 c} \operatorname{tgh} t)}{3 \sqrt{1-2 c}},
\end{aligned}
$$

and hence

$$
M_{331}=-\left.\widetilde{M}_{331}\right|_{-\infty} ^{0}=-\left.\frac{4 T_{0} e^{\sqrt{1-2 c} t}(3-2 c-3 \sqrt{1-2 c} \operatorname{tgh} t)}{3 \sqrt{1-2 c}}\right|_{-\infty} ^{0}=-\frac{4 T_{0}(3-2 c)}{3 \sqrt{1-2 c}} .
$$

For $v_{*}>\sqrt{2} / 2$, one obtains

$$
M_{331}=-\left.\widetilde{M}_{331}\right|_{-\infty} ^{t_{1}}+\left.\widetilde{M}_{331}\right|_{t_{1}} ^{t_{2}}-\left.\widetilde{M}_{331}\right|_{t_{2}} ^{0}=2\left(\widetilde{M}_{331}\left(t_{2}\right)-\widetilde{M}_{331}\left(t_{1}\right)\right)-\widetilde{M}_{331}(0)
$$

where $t_{1}, t_{2}$ are defined as earlier. According to the latter formulas and (2.159), one gets

$$
\begin{aligned}
M_{331}=-4 T_{0}( & \frac{3-2 c}{3 \sqrt{1-2 c}}+2\left(\frac{3-2 c}{3 \sqrt{1-2 c}}+\sqrt{1-x_{1}}\right)\left(\frac{\sqrt{x_{1}}}{1+\sqrt{1-x_{1}}}\right)^{\sqrt{1-2 c}} \\
& \left.-2\left(\frac{3-2 c}{3 \sqrt{1-2 c}}+\sqrt{1-x_{2}}\right)\left(\frac{\sqrt{x_{2}}}{1+\sqrt{1-x_{2}}}\right)^{\sqrt{1+2 c}}\right) .
\end{aligned}
$$

Since $1-x_{1,2}=x_{2,1}$, one gets

$$
\begin{aligned}
M_{331}=-4 T_{0}( & \frac{3-2 c}{3 \sqrt{1-2 c}}+2\left(\frac{3-2 c}{3 \sqrt{1-2 c}}+\sqrt{x_{2}}\right)\left(\frac{\sqrt{x_{1}}}{1+\sqrt{x_{2}}}\right)^{\sqrt{1-2 c}} \\
& \left.-2\left(\frac{3-2 c}{3 \sqrt{1-2 c}}+\sqrt{x_{1}}\right)\left(\frac{\sqrt{x_{2}}}{1+\sqrt{x_{1}}}\right)^{\sqrt{1-2 c}}\right) .
\end{aligned}
$$

This part of our consideration provides the following main result:

$$
M_{331}=-4 T_{0} \frac{3-2 c}{3 \sqrt{1-2 c}}- \begin{cases}8 T_{0}\left(\left(\frac{3-2 c}{3 \sqrt{1-2 c}}+\sqrt{x_{2}}\right)\left(\frac{\sqrt{x_{1}}}{1+\sqrt{x_{2}}}\right)^{\sqrt{1-2 c}}\right. & \text { for } v_{*}<\frac{\sqrt{2}}{2}, \\ 0 & \left.-\left(\frac{3-2 c}{3 \sqrt{1-2 c}}+\sqrt{x_{1}}\right)\left(\frac{\sqrt{x_{2}}}{1+\sqrt{x_{1}}}\right)^{\sqrt{1-2 c}}\right) \\ \text { for } v_{*} \geq \frac{\sqrt{2}}{2} . \\ \text { (2.164) }\end{cases}
$$

Formula $M_{232}$ is defined in the following way:

$$
M_{332}=\left.\frac{4 v_{*}\left(1-\beta v_{*}^{2}\right)(3-2 c-3 \sqrt{1-2 c} \operatorname{tgh} t) \exp (\sqrt{1-2 c} t)}{3 \sqrt{1-2 c}}\right|_{-\infty} ^{0},
$$


34 Stick-slip chaos in a self-excited oscillator

and hence

$$
M_{332}=\frac{4 v_{*}\left(1-\beta v_{*}^{2}\right)(3-2 c)}{3 \sqrt{1-2 c}} .
$$

On the other hand, integral $M_{333}$ is as follows:

$$
M_{333}=\left.\frac{4 \sqrt{2}}{3}\left(3 \beta v_{*}^{2}-\alpha\right)\left(-2+\operatorname{sech}^{2} t+\sqrt{1-2 c} \operatorname{tgh} t\right) \exp (\sqrt{1-2 c} t) \operatorname{sech} t\right|_{-\infty} ^{0},
$$

and hence

$$
M_{333}=\frac{4 \sqrt{2}}{3}\left(3 \beta v_{*}^{2}-\alpha\right) .
$$

Formula $M_{334}$ is defined as follows:

$$
\begin{aligned}
M_{334}= & \frac{256 \beta v_{*}}{(2+\sqrt{1-2 c})(4+\sqrt{1-2 c})(6+\sqrt{1-2 c})(8+\sqrt{1-2 c})(10+\sqrt{1-2 c})} \\
& \times\left(\left(4 c^{2}+297(1+\sqrt{1-2 c})+6 c(46+7 \sqrt{1-2 c})\right) F\left(6,1+\frac{1}{2} \sqrt{1-2 c} ; 4+\frac{1}{2} \sqrt{1-2 c} ;-1\right)\right. \\
& +(-2 c-3)\left((42-4 c+24 \sqrt{1-2 c}) F\left(6,2+\frac{1}{2} \sqrt{1-2 c} ; 5+\frac{1}{2} \sqrt{1-2 c} ;-1\right)\right. \\
& \left.\left.+(-2 c-3+3 \sqrt{1-2 c}) F\left(6,3+\frac{1}{2} \sqrt{1-2 c} ; 6+\frac{1}{2} \sqrt{1-2 c} ;-1\right)\right)\right) .
\end{aligned}
$$

Integral $M_{335}$ is given below:

$$
\begin{aligned}
M_{335}=- & \frac{2048 \sqrt{2} \beta}{(3+\sqrt{1-2 c})(5+\sqrt{1-2 c})(7+\sqrt{1-2 c})(9+\sqrt{1-2 c})(11+\sqrt{1-2 c})(13+\sqrt{1-2 c})} \\
\times & \left(\left(2 c^{2}+252(1+\sqrt{1-2 c})-3 c(73+9 \sqrt{1-2 c})\right)\right. \\
& F\left(8, \frac{1}{2}(3+\sqrt{1-2 c}) ; \frac{1}{2}(11+\sqrt{1-2 c}) ;-1\right) \\
& +2(-2 c-3)\left((20-c+8 \sqrt{1-2 c}) F\left(8, \frac{1}{2}(5+\sqrt{1-2 c}) ; \frac{1}{2}(13+\sqrt{1-2 c}) ;-1\right)\right. \\
& +\left(2 c^{2}(-5 c-12)(-1+\sqrt{1-2 c})\right) \\
& \left.\left.F\left(8, \frac{1}{2}(7+\sqrt{1-2 c}) ; \frac{1}{2}(15+\sqrt{1-2 c}) ;-1\right)\right)\right) .
\end{aligned}
$$


Substitution of (2.163), (2.166), and (2.168) into (2.156) gives

$$
\begin{aligned}
& M_{33}=\frac{4(3+2 c)}{3 \sqrt{1+2 c}}\left(v_{*}\left(1-\beta v_{*}^{2}\right)-T_{0}\right)-\frac{4 \sqrt{2}}{3}\left(3 \beta v_{*}^{2}-\alpha\right) \\
& -M_{334}-M_{335}- \begin{cases}8 T_{0}\left(\left(\frac{3+2 c}{3 \sqrt{1+2 c}}+\sqrt{x_{2}}\right)\left(\frac{1+\sqrt{x_{2}}}{\sqrt{x_{1}}}\right)^{-\sqrt{1+2 c}}\right. & \\
\left.-\left(\frac{3+2 c}{3 \sqrt{1+2 c}}+\sqrt{x_{1}}\right)\left(\frac{1+\sqrt{x_{1}}}{\sqrt{x_{2}}}\right)^{-\sqrt{1+2 c}}\right) & \text { for } v_{*}<\frac{\sqrt{2}}{2}, \\
0 & \text { for } v_{*} \geq \frac{\sqrt{2}}{2} .\end{cases}
\end{aligned}
$$

Substituting (2.150), (2.155), and (2.171) into (2.144), we can define the following Melnikov function:

$$
\begin{aligned}
& M_{3}\left(t_{0}\right)=-\tilde{A} \sin \left(\omega t_{0}+\kappa\right)+\frac{4(3-2 c)}{3 \sqrt{1-2 c}}\left(v_{*}\left(1-\beta v_{*}^{2}\right)-T_{0}\right)+\frac{2 \sqrt{2}}{3}\left(\delta_{11}+\delta_{21}+2\left(3 \beta v_{*}^{2}-\alpha\right)\right) \\
&+\frac{\sqrt{2}}{3}\left(\alpha_{21}+\alpha_{11}\right)\left(3 \sqrt{1-2 c}-c \psi\left(\frac{1}{4}(1+\sqrt{1-2 c})\right)+c \psi\left(\frac{1}{4}(3+\sqrt{1-2 c})\right)\right) \\
&-M_{323}-M_{324} \\
&-M_{334}-M_{335}-\left\{\begin{array}{c}
8 T_{0}\left(\left(\frac{3-2 c}{3 \sqrt{1-2 c}}+\sqrt{x_{2}}\right)\left(\frac{\sqrt{x_{1}}}{1+\sqrt{x_{2}}}\right)^{\sqrt{1-2 c}}\right. \\
\left.-\left(\frac{3-2 c}{3 \sqrt{1-2 c}}+\sqrt{x_{1}}\right)\left(\frac{\sqrt{x_{2}}}{1+\sqrt{x_{1}}}\right)^{\sqrt{1-2 c}}\right)
\end{array} \text { for } v_{*}<\frac{\sqrt{2}}{2},\right. \\
& 0 \quad \text { for } v_{*} \geq \frac{\sqrt{2}}{2} .
\end{aligned}
$$

The condition of stable and unstable manifolds intersection is defined by the formula

$$
\tilde{A}>\mid \begin{aligned}
& \frac{4(3-2 c)}{3 \sqrt{1-2 c}}\left(v_{*}\left(1-\beta v_{*}^{2}\right)-T_{0}\right)+\frac{2 \sqrt{2}}{3}\left(\delta_{11}+\delta_{21}+2\left(3 \beta v_{*}^{2}-\alpha\right)\right) \\
& +\frac{\sqrt{2}}{3}\left(\alpha_{21}+\alpha_{11}\right)\left(3 \sqrt{1-2 c}-c \psi\left(\frac{1}{4}(1+\sqrt{1-2 c})\right)+c \psi\left(\frac{1}{4}(3+\sqrt{1-2 c})\right)\right)-M_{323}-M_{324}
\end{aligned}
$$




$$
-M_{334}-M_{335}-\left\{\begin{array}{ll}
8 T_{0}\left(\left(\frac{3-2 c}{3 \sqrt{1-2 c}}+\sqrt{x_{2}}\right)\left(\frac{\sqrt{x_{1}}}{1+\sqrt{x_{2}}}\right)^{\sqrt{1-2 c}}\right. & \\
\left.-\left(\frac{3-2 c}{3 \sqrt{1-2 c}}+\sqrt{x_{1}}\right)\left(\frac{\sqrt{x_{2}}}{1+\sqrt{x_{1}}}\right)^{\sqrt{1-2 c}}\right) & \text { for } v_{*}<\frac{\sqrt{2}}{2} \\
0 & \text { for } v_{*} \geq \frac{\sqrt{2}}{2}
\end{array} \mid .\right.
$$

The Melnikov function, according to (2.69), is defined in the following way:

$$
M_{1-}\left(t_{0}\right)=M_{11-}\left(t_{0}\right)-M_{12-},
$$

where

$$
\begin{gathered}
M_{11-}\left(t_{0}\right)=2 \gamma \int_{0}^{\infty} P(-t) \exp (-\sqrt{1-2 c} t) \cos \omega\left(t+t_{0}\right) d t \\
M_{12-}=2 \int_{0}^{\infty}\left(-\delta_{1}\left(x_{10}(t), \dot{x}_{10}(t)\right)+\delta_{2}\left(x_{10}(t), \dot{x}_{10}(t)\right)\right) P(-t) \exp (-\sqrt{1-2 c} t) d t
\end{gathered}
$$

Comparing the obtained formulas with (2.101), we get

$$
\begin{gathered}
M_{11-}\left(t_{0}\right)=M_{11}\left(t_{0}\right), \\
M_{12-}=M_{12} .
\end{gathered}
$$

Taking into account (2.106) and (2.112) in (2.174), we have

$$
\begin{aligned}
M_{1}\left(t_{0}\right)= & \tilde{A} \sin \left(\omega t_{0}-\kappa\right)-\frac{2 \sqrt{2}}{3}\left(\delta_{11}-\delta_{21}\right)+M_{123}+M_{124} \\
& -\frac{\sqrt{2}}{3}\left(\alpha_{21}-\alpha_{11}\right)\left(3 \sqrt{1-2 c}-c \psi\left(\frac{1}{4}(1+\sqrt{1-2 c})\right)+c \psi\left(\frac{1}{4}(3+\sqrt{1-2 c})\right)\right) .
\end{aligned}
$$

The criterion of chaos occurrence is governed by the following inequality:

$$
\begin{aligned}
\tilde{A}>\mid & -\frac{2 \sqrt{2}}{3}\left(\delta_{11}-\delta_{21}\right)+M_{123}+M_{124} \\
& -\frac{\sqrt{2}}{3}\left(\alpha_{21}-\alpha_{11}\right)\left(3 \sqrt{1-2 c}-c \psi\left(\frac{1}{4}(1+\sqrt{1-2 c})\right)+c \psi\left(\frac{1}{4}(3+\sqrt{1-2 c})\right)\right) \mid .
\end{aligned}
$$

According to (2.73), the Melnikov function is

$$
M_{2-}\left(t_{0}\right)=M_{21-}\left(t_{0}\right)+M_{22-}-M_{23-},
$$


where

$$
\begin{gathered}
M_{21-}\left(t_{0}\right)=2 A \gamma \int_{-\infty}^{\infty} \dot{r}(t) \cos \omega\left(t+t_{0}\right) d t, \\
M_{22-}=2 A \int_{-\infty}^{\infty}\left(\delta_{1}\left(x_{10}, \dot{x}_{10}\right)+\delta_{2}\left(x_{10}, \dot{x}_{10}\right)\right) \dot{r}(t) d t \\
M_{23-}=4 A \int_{-\infty}^{\infty} T\left(-\dot{x}_{01}-v_{*}\right) \dot{r}(t) d t=-4 A \int_{-\infty}^{\infty} T\left(-\dot{x}_{01}-v_{*}\right) \operatorname{sech} t \operatorname{tgh} t d t .
\end{gathered}
$$

Comparison of the obtained formulas with (2.116) gives

$$
\begin{gathered}
M_{21-}\left(t_{0}\right)=M_{21}\left(t_{0}\right), \\
M_{22-}=M_{22} .
\end{gathered}
$$

Using (2.5), the formula for $M_{23}$ - takes the form

$$
M_{23-}=-M_{231-}-M_{232-}-M_{233-}+M_{234-}-M_{235-},
$$

where the following notation is applied:

$$
\begin{aligned}
& M_{231-}=4 A T_{0} \int_{-\infty}^{\infty} \operatorname{sgn}\left(-\dot{x}_{01}(t)-v_{*}\right) \operatorname{sech} t \operatorname{tgh} t d t \\
& M_{232-}=4 A\left(v_{*}-\beta v_{*}^{3}\right) \int_{-\infty}^{\infty} \operatorname{sech} t \operatorname{tgh} t d t d t \\
& M_{233-}=4 \sqrt{2} A\left(3 \beta v_{*}^{2}-\alpha\right) \int_{-\infty}^{\infty} \operatorname{sech}^{2} t \operatorname{tgh}^{2} t d t \\
& M_{234-}=12 \sqrt{2} A \beta v_{*} \int_{-\infty}^{\infty} \operatorname{sech}^{3} t \operatorname{tgh}^{3} t d t \\
& M_{235-}=4 \sqrt{2} A \beta \int_{-\infty}^{\infty} \operatorname{sech}^{4} t \operatorname{tgh}^{4} t d t
\end{aligned}
$$

Computing integral $M_{231-}$ for $\left|v_{*}\right|>\sqrt{2} / 2$, one gets

$$
M_{231-}=-4 A T_{0} \int_{-\infty}^{\infty} \operatorname{sech} t \operatorname{tgh} t d t=0
$$

In the case $\left|v_{*}\right| \leq \sqrt{2} / 2$, we have

$$
M_{231-}=4 A T_{0}\left(-\int_{-\infty}^{t_{3}} \operatorname{sech} t \operatorname{tgh} t d t+\int_{t_{3}}^{t_{4}} \operatorname{sech} t \operatorname{tgh} t d t-\int_{t_{4}}^{\infty} \operatorname{sech} t \operatorname{tgh} t d t\right),
$$

where $t_{3}=-t_{2}, t_{4}=-t_{1}$. Upon integration we have

$$
\begin{aligned}
M_{231-} & =4 A T_{0}\left(\left.\operatorname{sech} t\right|_{-\infty} ^{t_{3}}-\left.\operatorname{sech} t\right|_{t_{3}} ^{t_{4}}+\left.\operatorname{sech} t\right|_{t_{4}} ^{\infty}\right) \\
& =8 A T_{0}\left(\operatorname{sech} t_{3}-\operatorname{sech} t_{4}\right)=-8 A T_{0}\left(\operatorname{sech} t_{1}-\operatorname{sech} t_{2}\right),
\end{aligned}
$$


and hence

$$
M_{231-}=-M_{231}=-8 A T_{0}\left(\sqrt{\frac{1}{2}+\sqrt{\frac{1}{4}-\frac{v_{*}^{2}}{2}}}-\sqrt{\frac{1}{2}-\sqrt{\frac{1}{4}-\frac{v_{*}^{2}}{2}}}\right) .
$$

Finally, we obtain

$$
M_{231-}= \begin{cases}-8 A T_{0}\left(\sqrt{\frac{1}{2}+\sqrt{\frac{1}{4}-\frac{v_{*}^{2}}{2}}}-\sqrt{\left.\frac{1}{2}-\sqrt{\frac{1}{4}-\frac{v_{*}^{2}}{2}}\right)}\right. & \text { for } v_{*}<\frac{\sqrt{2}}{2}, \\ 0 & \text { for } v_{*} \geq \frac{\sqrt{2}}{2} .\end{cases}
$$

Observe that

$$
M_{232-}=M_{234-}=0,
$$

and comparing (2.185) and (2.187) with (2.127) and (2.129), respectively, one gets

$$
\begin{gathered}
M_{233-}=M_{233}=\frac{8 \sqrt{2}}{3} A\left(3 \beta v_{*}^{2}-\alpha\right), \\
M_{235-}=M_{235}=\frac{16 \sqrt{2} A \beta}{35} .
\end{gathered}
$$

Substituting (2.192)-(2.194) into (2.182), we get

$$
\begin{aligned}
M_{23-}= & -\frac{8 \sqrt{2}}{3} A\left(3 \beta v_{*}^{2}-\alpha\right)-\frac{16 \sqrt{2} A \beta}{35} \\
& + \begin{cases}8 A T_{0}\left(\sqrt{\frac{1}{2}+\sqrt{\frac{1}{4}-\frac{v_{*}^{2}}{2}}}-\sqrt{\frac{1}{2}-\sqrt{\frac{1}{4}-\frac{v_{*}^{2}}{2}}}\right) & \text { for } v_{*}<\frac{\sqrt{2}}{2}, \\
0 & \text { for } v_{*} \geq \frac{\sqrt{2}}{2} .\end{cases}
\end{aligned}
$$

Substituting (2.119), (2.123), and (2.195) into (2.179), we get the following Melnikov function:

$$
\begin{aligned}
M_{2-}\left(t_{0}\right)= & 2 \pi A \gamma \omega \operatorname{sech}\left(\frac{\pi \omega}{2}\right) \sin \omega t_{0}+4 A\left(\frac{\sqrt{2}}{3}\left(\delta_{11}+\delta_{21}\right)+\frac{4}{15}\left(\delta_{12}+\delta_{22}\right)+\frac{4}{35} \sqrt{2}\left(\delta_{13}+\delta_{23}\right)\right) \\
& +\frac{8 \sqrt{2}}{3} A\left(3 \beta v_{*}^{2}-\alpha\right)+\frac{16 \sqrt{2} A \beta}{35} \\
& - \begin{cases}8 A T_{0}\left(\sqrt{\frac{1}{2}+\sqrt{\frac{1}{4}-\frac{v_{*}^{2}}{2}}}-\sqrt{\frac{1}{2}-\sqrt{\frac{1}{4}-\frac{v_{*}^{2}}{2}}}\right) & \text { for } v_{*}<\frac{\sqrt{2}}{2}, \\
0 & \text { for } v_{*} \geq \frac{\sqrt{2}}{2} .\end{cases}
\end{aligned}
$$


Stable and unstable manifolds intersection is defined in the following way:

$$
\begin{aligned}
& 2 \pi \gamma \omega|A| \operatorname{sech}\left(\frac{\pi \omega}{2}\right)>\mid 4 A\left(\frac{\sqrt{2}}{3}\left(\delta_{11}+\delta_{21}\right)+\frac{4}{15}\left(\delta_{12}+\delta_{22}\right)+\frac{4}{35} \sqrt{2}\left(\delta_{13}+\delta_{23}\right)\right) \\
& +\frac{8 \sqrt{2}}{3} A\left(3 \beta v_{*}^{2}-\alpha\right)+\frac{16 \sqrt{2} A \beta}{35} \\
& -\left\{\begin{array}{ll}
8 A T_{0}\left(\sqrt{\frac{1}{2}+\sqrt{\frac{1}{4}-\frac{v_{*}^{2}}{2}}}-\sqrt{\left.\frac{1}{2}-\sqrt{\frac{1}{4}-\frac{v_{*}^{2}}{2}}\right)}\right. & \text { for } v_{*}<\frac{\sqrt{2}}{2} \\
0 & \text { for } v_{*} \geq \frac{\sqrt{2}}{2}
\end{array} .\right.
\end{aligned}
$$

The Melnikov function (taking into account (2.76)) is defined as follows:

$$
M_{3-}\left(t_{0}\right)=-M_{31-}\left(t_{0}\right)-M_{32-}+M_{33-}
$$

where

$$
\begin{gathered}
M_{31-}\left(t_{0}\right)=M_{31}\left(t_{0}\right)=2 \gamma \int_{-\infty}^{0} P(t) \exp (\sqrt{1-2 c} t) \cos \omega\left(t+t_{0}\right) d t \\
M_{32-}=M_{32}=2 \int_{-\infty}^{0}\left(\delta_{1}\left(x_{10}(t), \dot{x}_{10}(t)\right)+\delta_{2}\left(x_{10}(t), \dot{x}_{10}(t)\right)\right) P(t) \exp (\sqrt{1-2 c} t) d t \\
M_{33-}=-M_{33}=4 \int_{-\infty}^{0} T\left(-\dot{x}_{01}-v_{*}\right) P(t) \exp (\sqrt{1-2 c} t) d t
\end{gathered}
$$

Using (2.5), formula $M_{33-}$ takes the form

$$
M_{33-}=M_{331-}+M_{332-}+M_{333-}-M_{334-}+M_{335-},
$$

where the following notation is applied:

$$
\begin{aligned}
& M_{331-}=4 T_{0} \int_{-\infty}^{0} \operatorname{sgn}\left(-\dot{x}_{10}(t)-v_{*}\right)\left(-\frac{2}{3} c-(\sqrt{1-2 c}-\operatorname{tgh} t) \operatorname{tgh} t\right) \exp (\sqrt{1-2 c} t) d t, \\
& M_{332-}=4 v_{*}\left(1-\beta v_{*}^{2}\right) \int_{-\infty}^{0}\left(-\frac{2}{3} c-(\sqrt{1-2 c}-\operatorname{tgh} t) \operatorname{tgh} t\right) \exp (\sqrt{1-2 c} t) d t, \\
& M_{333-}=4 \sqrt{2}\left(3 \beta v_{*}^{2}-\alpha\right) \int_{-\infty}^{0}\left(-\frac{2}{3} c-(\sqrt{1-2 c}-\operatorname{tgh} t) \operatorname{tgh} t\right) \exp (\sqrt{1-2 c} t) \operatorname{sech} t \operatorname{tgh} t d t,
\end{aligned}
$$




$$
\begin{aligned}
& M_{334-}=24 \beta v_{*} \int_{-\infty}^{0}\left(-\frac{2}{3} c-(\sqrt{1-2 c}-\operatorname{tgh} t) \operatorname{tgh} t\right) \exp (\sqrt{1-2 c} t) \operatorname{sech}^{2} t \operatorname{tgh}^{2} t d t, \\
& M_{335-}=8 \sqrt{2} \beta \int_{-\infty}^{0}\left(-\frac{2}{3} c-(\sqrt{1-2 c}-\operatorname{tgh} t) \operatorname{tgh} t\right) \exp (\sqrt{1-2 c} t) \operatorname{sech}^{3} t \operatorname{tgh}^{3} t d t .
\end{aligned}
$$

Since for $-\infty<t \leq 0$ we have $\operatorname{sgn}\left(-\dot{x}_{10}(t)-v_{*}\right)=-1$, one gets

$$
M_{331-}=-4 T_{0} \int_{-\infty}^{0}\left(-\frac{2}{3} c-(\sqrt{1-2 c}-\operatorname{tgh} t) \operatorname{tgh} t\right) \exp (\sqrt{1-2 c} t) d t .
$$

\section{Integration gives}

$$
\begin{aligned}
M_{331-}= & M_{331}=-\frac{4 T_{0}(3-2 c)}{3 \sqrt{1-2 c}}, \\
M_{332-}= & M_{332}=\frac{4 v_{*}\left(1-\beta v_{*}^{2}\right)(3-2 c)}{3 \sqrt{1-2 c}}, \\
M_{333-}= & M_{333}=\frac{4 \sqrt{2}}{3}\left(3 \beta v_{*}^{2}-\alpha\right), \\
M_{334-}= & M_{334}=\frac{256 \beta v_{*}}{(2+\sqrt{1-2 c})(4+\sqrt{1-2 c})(6+\sqrt{1-2 c})(8+\sqrt{1-2 c})(10+\sqrt{1-2 c})} \\
& \times\left(\left(4 c^{2}+297(1+\sqrt{1-2 c})+6 c(46+7 \sqrt{1-2 c})\right) F\left(6,1+\frac{1}{2} \sqrt{1-2 c} ; 4+\frac{1}{2} \sqrt{1-2 c} ;-1\right)\right. \\
& +(-2 c-3)\left((42-4 c+24 \sqrt{1-2 c}) F\left(6,2+\frac{1}{2} \sqrt{1-2 c} ; 5+\frac{1}{2} \sqrt{1-2 c} ;-1\right)\right. \\
M_{335-}= & \left.\left.M_{335} \quad+(-2 c-3+3 \sqrt{1-2 c}) F\left(6,3+\frac{1}{2} \sqrt{1-2 c} ; 6+\frac{1}{2} \sqrt{1-2 c} ;-1\right)\right)\right), \\
=- & \frac{2048 \sqrt{2} \beta}{(3+\sqrt{1-2 c})(5+\sqrt{1-2 c})(7+\sqrt{1-2 c})(9+\sqrt{1-2 c})(11+\sqrt{1-2 c})(13+\sqrt{1-2 c})} \\
\times & \left(\left(2 c^{2}+252(1+\sqrt{1-2 c})-3 c(73+9 \sqrt{1-2 c})\right)\right. \\
& F\left(8, \frac{1}{2}(3+\sqrt{1-2 c}) ; \frac{1}{2}(11+\sqrt{1-2 c}) ;-1\right)+2(-2 c-3) \\
& \quad\left((20-c+8 \sqrt{1-2 c}) F\left(8, \frac{1}{2}(5+\sqrt{1-2 c}) ; \frac{1}{2}(13+\sqrt{1-2 c}) ;-1\right)\right. \\
& \quad+\left(2 c^{2}(-5 c-12)(-1+\sqrt{1-2 c})\right) \\
& \left.\left.F\left(8, \frac{1}{2}(7+\sqrt{1-2 c}) ; \frac{1}{2}(15+\sqrt{1-2 c}) ;-1\right)\right)\right) .
\end{aligned}
$$

Substituting (2.203) into (2.200), one gets

$$
M_{33}=\frac{4(3-2 c)}{3 \sqrt{1-2 c}}\left(v_{*}\left(1-\beta v_{*}^{2}\right)-T_{0}\right)-\frac{4 \sqrt{2}}{3}\left(3 \beta v_{*}^{2}-\alpha\right)-M_{334}-M_{335} .
$$


Substitution of (2.150), (2.155), and (2.204) into (2.144) gives the Melnikov function defined as follows:

$$
\begin{aligned}
M_{3-}\left(t_{0}\right)= & -\widetilde{A} \sin \left(\omega t_{0}+\kappa\right)+\frac{4(3-2 c)}{3 \sqrt{1-2 c}}\left(v_{*}\left(1-\beta v_{*}^{2}\right)-T_{0}\right)+\frac{2 \sqrt{2}}{3}\left(\delta_{11}+\delta_{21}+2\left(3 \beta v_{*}^{2}-\alpha\right)\right) \\
& +\frac{\sqrt{2}}{3}\left(\alpha_{21}+\alpha_{11}\right)\left(3 \sqrt{1-2 c}-c \psi\left(\frac{1}{4}(1+\sqrt{1-2 c})\right)+c \psi\left(\frac{1}{4}(3+\sqrt{1-2 c})\right)\right) \\
& -M_{323}-M_{324}-M_{334}-M_{335} .
\end{aligned}
$$

The chaos criterion is

$$
\begin{aligned}
\tilde{A}>\mid & \frac{4(3-2 c)}{3 \sqrt{1-2 c}}\left(v_{*}\left(1-\beta v_{*}^{2}\right)-T_{0}\right)+\frac{2 \sqrt{2}}{3}\left(\delta_{11}+\delta_{21}+2\left(3 \beta v_{*}^{2}-\alpha\right)\right) \\
& +\frac{\sqrt{2}}{3}\left(\alpha_{21}+\alpha_{11}\right)\left(3 \sqrt{1-2 c}-c \psi\left(\frac{1}{4}(1+\sqrt{1-2 c})\right)+c \psi\left(\frac{1}{4}(3+\sqrt{1-2 c})\right)\right) \\
& -M_{323}-M_{324}-M_{334}-M_{335} \mid .
\end{aligned}
$$

According to (2.81), the Melnikov function is

$$
N_{1}\left(t_{0}\right)=N_{11}\left(t_{0}\right)-N_{12}-N_{13}+N_{14}
$$

where

$$
\begin{aligned}
N_{11}\left(t_{0}\right) & =2 \gamma \int_{0}^{\infty} R(-\sqrt{1-2 c} t) e^{-t} \cos \omega\left(t+t_{0}\right) d t \\
& =2 \gamma \int_{0}^{\infty}\left(\frac{3-4 c}{3(1-2 c)}-\operatorname{sech}^{2}(-\sqrt{1-2 c} t)-\frac{\operatorname{tgh}(-\sqrt{1-2 c} t)}{\sqrt{1-2 c}}\right) e^{-t} \cos \omega\left(t+t_{0}\right) d t \\
N_{12} & =2 \int_{0}^{\infty}\left(\delta_{1}\left(x_{10}(t), \dot{x}_{10}(t)\right)-\delta_{2}\left(x_{10}(t), \dot{x}_{10}(t)\right)\right) e^{-t} R(-\sqrt{1-2 c} t) d t \\
N_{13} & =2 \int_{0}^{\infty} e^{-t} R(-\sqrt{1-2 c} t) T\left(\dot{x}_{01}-v_{*}\right) d t \\
& =2 \int_{0}^{\infty} e^{-t}\left(\frac{3-4 c}{3(1-2 c)}-\operatorname{sech}^{2}(-\sqrt{1-2 c} t)-\frac{\operatorname{tgh}(-\sqrt{1-2 c} t)}{\sqrt{1-2 c}}\right) T\left(\dot{x}_{01}-v_{*}\right) d t \\
N_{14} & =2 \int_{0}^{\infty} e^{-t} R(-\sqrt{1-2 c} t) T\left(-\dot{x}_{01}-v_{*}\right) d t \\
& =2 \int_{0}^{\infty} e^{-t}\left(\frac{3-4 c}{3(1-2 c)}-\operatorname{sech}^{2}(-\sqrt{1-2 c} t)-\frac{\operatorname{tgh}(-\sqrt{1-2 c} t)}{\sqrt{1-2 c}}\right) T\left(-\dot{x}_{01}-v_{*}\right) d t .
\end{aligned}
$$


Formula $N_{11}\left(t_{0}\right)$ is defined as follows:

$$
N_{11}\left(t_{0}\right)=N_{111}\left(t_{0}\right)-N_{112}\left(t_{0}\right)
$$

where

$$
\begin{aligned}
& N_{111}\left(t_{0}\right)=2 \gamma \cos \omega t_{0} \int_{0}^{\infty}\left(\frac{3-4 c}{3(1-2 c)}-\operatorname{sech}^{2}(-\sqrt{1-2 c} t)-\frac{\operatorname{tgh}(-\sqrt{1-2 c} t)}{\sqrt{1-2 c}}\right) e^{-t} \cos \omega t d t \\
& N_{112}\left(t_{0}\right)=2 \gamma \sin \omega t_{0} \int_{0}^{\infty}\left(\frac{3-4 c}{3(1-2 c)}-\operatorname{sech}^{2}(-\sqrt{1-2 c} t)-\frac{\operatorname{tgh}(-\sqrt{1-2 c} t)}{\sqrt{1-2 c}}\right) e^{-t} \sin \omega t d t
\end{aligned}
$$

Upon integration we have

$$
\begin{aligned}
N_{112}\left(t_{0}\right)=\frac{\gamma \cos \omega t_{0}}{3(2 c-1) \sqrt{1-2 c}}( & -\frac{2 \omega((4 c-3) \sqrt{1-2 c}-3(2 c-1))}{1+\omega^{2}}+\frac{3}{2} \omega \sqrt{1-2 c} \\
\times\left(\psi\left(\frac{1+\omega i}{4 \sqrt{1-2 c}}\right)+\psi\left(\frac{1-\omega i}{4 \sqrt{1-2 c}}\right)-\psi\left(\frac{1}{4}\left(2+\frac{1+\omega i}{\sqrt{1-2 c}}\right)\right)\right. & \left.\left.-\psi\left(\frac{1}{4}\left(2+\frac{1-\omega i}{\sqrt{1-2 c}}\right)\right)\right)\right) .
\end{aligned}
$$

Owing to the property $\psi(\bar{z})=\overline{\psi(z)}$, one gets

$$
\begin{aligned}
N_{111}\left(t_{0}\right)=\gamma \cos \omega t_{0}( & \frac{6 \sqrt{1-2 c} \omega^{2}-2(4 c-3)}{3(2 c-1)\left(1+\omega^{2}\right)} \\
& \left.+\frac{\omega}{2 c-1}\left(\operatorname{Im} \psi\left(\frac{1+\omega i}{4 \sqrt{1-2 c}}\right)-\operatorname{Im} \psi\left(\frac{1}{4}\left(2+\frac{1+\omega i}{\sqrt{1-2 c}}\right)\right)\right)\right), \\
N_{112}\left(t_{0}\right)=\gamma \sin \omega t_{0}( & -\frac{2 \omega((4 c-3)+3 \sqrt{1-2 c})}{3(2 c-1)\left(1+\omega^{2}\right)} \\
& \left.+\frac{\omega}{2 c-1}\left(\operatorname{Re} \psi\left(\frac{1+\omega i}{4 \sqrt{1-2 c}}\right)-\operatorname{Re} \psi\left(\frac{1}{4}\left(2+\frac{1-\omega i}{\sqrt{1-2 c}}\right)\right)\right)\right) .
\end{aligned}
$$

Substitution of the above formulas into (2.209) gives

$$
N_{11}\left(t_{0}\right)=\gamma\left(A_{1} \cos \omega t_{0}-A_{2} \sin \omega t_{0}\right)=\tilde{A} \sin \left(\omega t_{0}-\kappa\right),
$$


where

$$
\begin{aligned}
& A_{1}=\frac{6 \sqrt{1-2 c} \omega^{2}-2(4 c-3)}{3(2 c-1)\left(1+\omega^{2}\right)}+\frac{\omega}{2 c-1}\left(\operatorname{Im} \psi\left(\frac{1+\omega i}{4 \sqrt{1-2 c}}\right)-\operatorname{Im} \psi\left(\frac{1}{4}\left(2+\frac{1+\omega i}{\sqrt{1-2 c}}\right)\right)\right), \\
& A_{2}=-\frac{2 \omega((4 c-3)+3 \sqrt{1-2 c})}{3(2 c-1)\left(1+\omega^{2}\right)}+\frac{\omega}{2 c-1}\left(\operatorname{Re} \psi\left(\frac{1+\omega i}{4 \sqrt{1-2 c}}\right)-\operatorname{Re} \psi\left(\frac{1}{4}\left(2+\frac{1-\omega i}{\sqrt{1-2 c}}\right)\right)\right),
\end{aligned}
$$

and $\tilde{A}=\gamma \sqrt{A_{1}^{2}+A_{2}^{2}}, \operatorname{tg} \kappa=A_{1} / A_{2}$.

The formula for $N_{12}$ is as follows:

$$
N_{12}=N_{121}-N_{122}-N_{123}-N_{124},
$$

where

$$
\begin{aligned}
N_{121}= & 2 \sqrt{2(1-2 c)}\left(\alpha_{11}-\alpha_{21}\right) \int_{0}^{\infty} \operatorname{sech}(\sqrt{1-2 c} t) e^{-t} R(-\sqrt{1-2 c} t) d t \\
N_{122}= & 2 \sqrt{2}(1-2 c)\left(\delta_{11}-\delta_{21}\right) \int_{0}^{\infty} \operatorname{sech}(\sqrt{1-2 c} t) \operatorname{tgh}(\sqrt{1-2 c} t) e^{-t} R(-\sqrt{1-2 c} t) d t \\
N_{123}= & 4(1-2 c)^{2}\left(\delta_{12}-\delta_{22}\right) \\
& \times \int_{0}^{\infty} \operatorname{sech}^{2}(\sqrt{1-2 c} t) \operatorname{tgh}^{2}(\sqrt{1-2 c} t) \\
& e^{-t} R(-\sqrt{1-2 c} t) \operatorname{sgn}(\operatorname{sech}(\sqrt{1-2 c} t) \operatorname{tgh}(\sqrt{1-2 c} t)) d t, \\
N_{124}= & 4 \sqrt{2}(1-2 c)^{3}\left(\delta_{13}-\delta_{23}\right) \int_{0}^{\infty} \operatorname{sech}^{3}(\sqrt{1-2 c} t) \operatorname{tgh}^{3}(\sqrt{1-2 c} t) e^{-t} R(-\sqrt{1-2 c} t) d t .
\end{aligned}
$$

Computations yield

$$
\begin{aligned}
N_{121}= & \frac{\sqrt{2}}{3(2 c-1)}\left(\alpha_{21}-\alpha_{11}\right)\left(3 \sqrt{1-2 c}+c \psi\left(\frac{1}{4}\left(1+\frac{1}{\sqrt{1-2 c}}\right)\right)-c \psi\left(\frac{1}{4}\left(3+\frac{1}{\sqrt{1-2 c}}\right)\right)\right) \\
N_{122}= & -\frac{2 \sqrt{2(1-2 c)}}{3}\left(\delta_{11}-\delta_{21}\right), \\
N_{123}= & -\frac{128(1-2 c)^{3 / 2}\left(\delta_{22}-\delta_{12}\right)}{3(1+2 \sqrt{1-2 c})(1+4 \sqrt{1-2 c})(1+6 \sqrt{1-2 c})(1+8 \sqrt{1-2 c})(1+10 \sqrt{1-2 c})} \\
& \times\left(\left(-6 a(191+152 \sqrt{1-2 c})+297(1+\sqrt{1-2 c})+16(69+40 \sqrt{1-2 c}) c^{2}\right)\right. \\
& \quad \times F\left(6, \frac{1}{2}\left(2+\frac{1}{\sqrt{1-2 c}}\right) ; \frac{1}{2}\left(8+\frac{1}{\sqrt{1-2 c}}\right) ;-1\right)
\end{aligned}
$$




$$
\begin{aligned}
& +(3+8 c)((-6(4+7 \sqrt{1-2 c})+16 c(3+5 \sqrt{1-2 c})) \\
& \times F\left(6, \frac{1}{2}\left(4+\frac{1}{\sqrt{1-2 c}}\right) ; \frac{1}{2}\left(10+\frac{1}{\sqrt{1-2 c}}\right) ;-1\right) \\
& +(-3+3 \sqrt{1-2 c}+6 c-8 c \sqrt{1-2 c}) \\
& \left.\left.F\left(6, \frac{1}{2}\left(6+\frac{1}{\sqrt{1-2 c}}\right) ; \frac{1}{2}\left(12+\frac{1}{\sqrt{1-2 c}}\right) ;-1\right)\right)\right) \\
& N_{124}=-\frac{1024 \sqrt{2}(1-2 c)^{3}\left(\delta_{23}-\delta_{13}\right)}{(1+3 \sqrt{1-2 c})(1+5 \sqrt{1-2 c})(1+7 \sqrt{1-2 c})(1+9 \sqrt{1-2 c})(1+11 \sqrt{1-2 c})(1+13 \sqrt{1-2 c})} \\
& \times\left(\left(252(1+\sqrt{1-2 c})-3 c(327+263 \sqrt{1-2 c})+(954+572 \sqrt{1-2 c}) c^{2}\right)\right. \\
& \times F\left(8, \frac{1}{2}\left(3+\frac{1}{\sqrt{1-2 c}}\right) ; \frac{1}{2}\left(11+\frac{1}{\sqrt{1-2 c}}\right) ;-1\right) \\
& +2(3+8 c)(-8-20 \sqrt{1-2 c}+16 c+39 c \sqrt{1-2 c}) \\
& F\left(8, \frac{1}{2}\left(5+\frac{1}{\sqrt{1-2 c}}\right) ; \frac{1}{2}\left(13+\frac{1}{\sqrt{1-2 c}}\right) ;-1\right) \\
& +\left(12(-1+\sqrt{1-2 c})-53 c(-1+\sqrt{1-2 c})+(-58+60 \sqrt{1-2 c}) c^{2}\right) \\
& \left.\times F\left(8, \frac{1}{2}\left(7+\frac{1}{\sqrt{1-2 c}}\right) ; \frac{1}{2}\left(15+\frac{1}{\sqrt{1-2 c}}\right) ;-1\right)\right),
\end{aligned}
$$

and hence

$$
\begin{aligned}
N_{12}= & \frac{\sqrt{2}}{3(2 c-1)}\left(\alpha_{21}-\alpha_{11}\right)\left(3 \sqrt{1-2 c}+c \psi\left(\frac{1}{4}\left(1+\frac{1}{\sqrt{1-2 c}}\right)\right)-c \psi\left(\frac{1}{4}\left(3+\frac{1}{\sqrt{1-2 c}}\right)\right)\right) \\
& +\frac{2 \sqrt{2(1-2 c)}}{3}\left(\delta_{11}-\delta_{21}\right)-N_{123}-N_{124} .
\end{aligned}
$$

According to (2.5), the formula for $N_{13}$ is

$$
N_{13}=N_{131}+N_{132}-N_{133}-N_{134}-N_{135} \text {, }
$$


where

$$
\begin{aligned}
& N_{131}=2 T_{0} \int_{0}^{\infty} e^{-t}\left(\frac{3-4 c}{3(1-2 c)}-\operatorname{sech}^{2}(-\sqrt{1-2 c} t)-\frac{\operatorname{tgh}(-\sqrt{1-2 c} t)}{\sqrt{1-2 c}}\right) \operatorname{sgn}\left(\dot{x}_{01}-v_{*}\right) d t, \\
& N_{132}=2\left(v_{*}-\beta v_{*}^{3}\right) \int_{0}^{\infty} e^{-t}\left(\frac{3-4 c}{3(1-2 c)}-\operatorname{sech}^{2}(-\sqrt{1-2 c} t)-\frac{\operatorname{tgh}(-\sqrt{1-2 c} t)}{\sqrt{1-2 c}}\right) d t, \\
& N_{133}=2 \sqrt{2}(1-2 c)\left(3 \beta v_{*}^{2}-\alpha\right)
\end{aligned}
$$$$
\times \int_{0}^{\infty} e^{-t}\left(\frac{3-4 c}{3(1-2 c)}-\operatorname{sech}^{2}(-\sqrt{1-2 c} t)-\frac{\operatorname{tgh}(-\sqrt{1-2 c} t)}{\sqrt{1-2 c}}\right)
$$$$
\operatorname{sech}(\sqrt{1-2 c} t) \operatorname{tgh}(\sqrt{1-2 c} t) d t
$$$$
N_{134}=12(1-2 c)^{2} \beta v_{*}
$$$$
\times \int_{0}^{\infty} e^{-t}\left(\frac{3-4 c}{3(1-2 c)}-\operatorname{sech}^{2}(-\sqrt{1-2 c} t)-\frac{\operatorname{tgh}(-\sqrt{1-2 c} t)}{\sqrt{1-2 c}}\right)
$$$$
\operatorname{sech}^{2}(\sqrt{1-2 c} t) \operatorname{tgh}^{2}(\sqrt{1-2 c} t) d t
$$$$
N_{135}=4 \sqrt{2}(1-2 c)^{3} \beta
$$

$$
\begin{aligned}
\times \int_{0}^{\infty} e^{-t}\left(\frac{3-4 c}{3(1-2 c)}-\operatorname{sech}^{2}(-\sqrt{1-2 c} t)-\frac{\operatorname{tgh}(-\sqrt{1-2 c} t)}{\sqrt{1-2 c}}\right) \\
\quad \operatorname{sech}^{3}(\sqrt{1-2 c} t) \operatorname{tgh}^{3}(\sqrt{1-2 c} t) d t .
\end{aligned}
$$

Now, computing integral $N_{131}$, it is easy to observe that for $t_{1}<0$ and $t_{2}<0$, we have $\operatorname{sgn}\left(\dot{x}_{01}-v_{*}\right)=-1$ irrespective of the value of velocity $v_{*}$ for $t \in(0, \infty)$. This observation leads to

$$
\begin{aligned}
N_{131} & =-2 T_{0} \int_{0}^{\infty} e^{-t}\left(\frac{3-4 c}{3(1-2 c)}-\operatorname{sech}^{2}(-\sqrt{1-2 c} t)-\frac{\operatorname{tgh}(-\sqrt{1-2 c} t)}{\sqrt{1-2 c}}\right) d t \\
& =-\left(\lim _{t \rightarrow \infty} \bar{N}_{131}(t)-\bar{N}_{131}(0)\right)=\frac{2 T_{0}(4 c-3)}{6 c-3},
\end{aligned}
$$


where

$$
\bar{N}_{131}(t)=\frac{2 T_{0} e^{-t}(3-4 c+3 \sqrt{1-2 c} \operatorname{tgh}(\sqrt{1-2 c} t))}{3(2 c-1)} .
$$

Integral $N_{132}$ is

$$
\begin{aligned}
N_{132} & =2\left(v_{*}-\beta v_{*}^{3}\right) \int_{0}^{\infty} e^{-t}\left(\frac{3-4 c}{3(1-2 c)}-\operatorname{sech}^{2}(-\sqrt{1-2 c} t)-\frac{\operatorname{tgh}(-\sqrt{1-2 c} t)}{\sqrt{1-2 c}}\right) d t \\
& =\frac{2(4 c-3)}{6 c-3}\left(v_{*}-\beta v_{*}^{3}\right) .
\end{aligned}
$$

On the other hand, integral $N_{133}$ is equal to

$$
\begin{aligned}
N_{133}= & \frac{\sqrt{2}(1-2 c)\left(3 \beta v_{*}^{2}-\alpha\right)}{6 c-3} \\
& \times\left. e^{-t} \operatorname{sech}^{3}(\sqrt{1-2 c} t)(\sinh (2 \sqrt{1-2 c} t)+2 \sqrt{1-2 c} t \cosh (2 \sqrt{1-2 c} t))\right|_{0} ^{\infty},
\end{aligned}
$$

and therefore

$$
N_{133}=\frac{2}{3} \sqrt{2(1-2 c)}\left(3 \beta v_{*}^{2}-\alpha\right)
$$

Integral $N_{134}$ is defined as follows:

$$
\begin{aligned}
N_{134}= & \frac{384(1-2 c)^{2} \beta v_{*}}{3 \sqrt{1-2 c}(1+2 \sqrt{1-2 c})(1+4 \sqrt{1-2 c})(1+6 \sqrt{1-2 c})(1+8 \sqrt{1-2 c})(1+10 \sqrt{1-2 c})} \\
\times & \left(\left(297(1+\sqrt{1-2 c})-6(191+152 \sqrt{1-2 c}) c+16(69+40 \sqrt{1-2 c}) c^{2}\right)\right. \\
& \times F\left(6,1+\frac{1}{2 \sqrt{1-2 c}} ; 4+\frac{1}{2 \sqrt{1-2 c}} ;-1\right) \\
& -(8 c-3)(-6(4+7 \sqrt{1-2 c})+80 c(1+\sqrt{1-2 c})) \\
& \cdot F\left(6,2+\frac{1}{2 \sqrt{1-2 c}} ; 5+\frac{1}{2 \sqrt{1-2 c}} ;-1\right) \\
& \left.+(3(\sqrt{1-2 c}-1)-2 c(4 \sqrt{1-2 c}-3)) F\left(6,3+\frac{1}{2 \sqrt{1-2 c}} ; 6+\frac{1}{2 \sqrt{1-2 c}} ;-1\right)\right)
\end{aligned}
$$


whereas integral $M_{135}$ is

$$
\begin{aligned}
N_{135}= & \frac{1024 \sqrt{2}(1-2 c)^{3} \beta}{(1+3 \sqrt{1-2 c})(1+5 \sqrt{1-2 c})(1+7 \sqrt{1-2 c})(1+9 \sqrt{1-2 c})(1+11 \sqrt{1-2 c})(1+13 \sqrt{1-2 c})} \\
\times & \left(252(1+\sqrt{1-2 c})-3(327+263 \sqrt{1-2 c}) c+(954+572 \sqrt{1-2 c}) c^{2}\right) \\
& \times F\left(8, \frac{1}{2}\left(3+\frac{1}{\sqrt{1-2 c}}\right) ; \frac{1}{2}\left(11+\frac{1}{\sqrt{1-2 c}}\right) ;-1\right) \\
& -2(8 c-3)(-8-20 \sqrt{1-2 c}+16 c+39 c \sqrt{1-2 c}) \\
& \cdot F\left(8, \frac{1}{2}\left(5+\frac{1}{\sqrt{1-2 c}}\right) ; \frac{1}{2}\left(13+\frac{1}{\sqrt{1-2 c}}\right) ;-1\right) \\
& +\left(12(\sqrt{1-2 c}-1)-53 c(\sqrt{1-2 c}-1)+c^{2}(60 \sqrt{1-2 c}-58)\right) \\
& \left.\times F\left(8, \frac{1}{2}\left(7+\frac{1}{\sqrt{1-2 c}}\right) ; \frac{1}{2}\left(15+\frac{1}{\sqrt{1-2 c}}\right) ;-1\right)\right) .
\end{aligned}
$$

Substitution of the above formulas into (2.221) gives

$$
N_{13}=\frac{2(4 c-3)}{6 c-3}\left(v_{*}-\beta v_{*}^{3}+T_{0}\right)-\frac{2}{3} \sqrt{2(1-2 c)}\left(3 \beta v_{*}^{2}-\alpha\right)-N_{134}-N_{135}
$$

Applying (2.5), the formula for $N_{14}$ takes the form

$$
N_{14}=N_{141}+N_{142}+N_{143}-N_{144}+N_{145}
$$

where

$$
\begin{aligned}
N_{141}= & 2 T_{0} \int_{0}^{\infty} e^{-t}\left(\frac{3-4 c}{3(1-2 c)}-\operatorname{sech}^{2}(-\sqrt{1-2 c} t)-\frac{\operatorname{tgh}(-\sqrt{1-2 c} t)}{\sqrt{1-2 c}}\right) \operatorname{sgn}\left(-\dot{x}_{01}-v_{*}\right) d t \\
N_{142}= & 2\left(v_{*}-\beta v_{*}^{3}\right) \int_{0}^{\infty} e^{-t}\left(\frac{3-4 c}{3(1-2 c)}-\operatorname{sech}^{2}(-\sqrt{1-2 c} t)-\frac{\operatorname{tgh}(-\sqrt{1-2 c} t)}{\sqrt{1-2 c}}\right) d t, \\
N_{143}= & 2 \sqrt{2}(1-2 c)\left(3 \beta v_{*}^{2}-\alpha\right) \\
& \times \int_{0}^{\infty} e^{-t}\left(\frac{3-4 c}{3(1-2 c)}-\operatorname{sech}^{2}(-\sqrt{1-2 c} t)-\frac{\operatorname{tgh}(-\sqrt{1-2 c} t)}{\sqrt{1-2 c}}\right) \\
& \operatorname{sech}(\sqrt{1-2 c} t) \operatorname{tgh}(\sqrt{1-2 c} t) d t
\end{aligned}
$$




$$
\begin{aligned}
N_{144}= & 12(1-2 c)^{2} \beta v_{*} \\
\times & \int_{0}^{\infty} e^{-t}\left(\frac{3-4 c}{3(1-2 c)}-\operatorname{sech}^{2}(-\sqrt{1-2 c} t)-\frac{\operatorname{tgh}(-\sqrt{1-2 c} t)}{\sqrt{1-2 c}}\right) \\
& \operatorname{sech}^{2}(\sqrt{1-2 c} t) \operatorname{tgh}^{2}(\sqrt{1-2 c} t) d t, \\
N_{145}= & 4 \sqrt{2}(1-2 c)^{3} \beta \\
& \times \int_{0}^{\infty} e^{-t}\left(\frac{3-4 c}{3(1-2 c)}-\operatorname{sech}^{2}(-\sqrt{1-2 c} t)-\frac{\operatorname{tgh}(-\sqrt{1-2 c} t)}{\sqrt{1-2 c}}\right) \\
& \operatorname{sech}^{3}(\sqrt{1-2 c} t) \operatorname{tgh}^{3}(\sqrt{1-2 c} t) d t .
\end{aligned}
$$

Integral $N_{141}$ for $\left|v_{*}\right|>(1-2 c) / \sqrt{2}$ is defined as follows:

$$
\begin{aligned}
N_{141} & =-2 T_{0} \int_{0}^{\infty} e^{-t}\left(\frac{3-4 c}{3(1-2 c)}-\operatorname{sech}^{2}(-\sqrt{1-2 c} t)-\frac{\operatorname{tgh}(-\sqrt{1-2 c} t)}{\sqrt{1-2 c}}\right) d t \\
& =-\left(\lim _{t \rightarrow \infty} \bar{N}_{141}(t)-\bar{N}_{141}(0)\right)=\frac{2 T_{0}(4 c-3)}{6 c-3},
\end{aligned}
$$

where

$$
\bar{N}_{141}(t)=\frac{2 T_{0} e^{-t}(3-4 c+3 \sqrt{1-2 c} \operatorname{tgh}(\sqrt{1-2 c} t))}{3(2 c-1)} .
$$

In the second case, that is, for $\left|v_{*}\right|<(1-2 c) / \sqrt{2}$, one obtains

$$
\begin{aligned}
N_{141} & =2 T_{0}\left(-\left.\bar{N}_{141}(t)\right|_{0} ^{t_{3}}+\left.\bar{N}_{141}(t)\right|_{t_{3}} ^{t_{4}}-\left.\bar{N}_{141}(t)\right|_{t_{4}} ^{\infty}\right) \\
& =-2 T_{0}\left(2\left(\bar{N}_{141}\left(t_{3}\right)-\bar{N}_{141}\left(t_{4}\right)\right)+\lim _{t \rightarrow \infty} \bar{N}_{141}(t)-\bar{N}_{141}(0)\right) .
\end{aligned}
$$

Taking into account (2.233), one obtains

$$
N_{141}=-2 T_{0}\left(2\left(\bar{N}_{141}\left(t_{3}\right)-\bar{N}_{141}\left(t_{4}\right)\right)-\frac{4 c-3}{6 c-3}\right) .
$$

This part of our consideration provides the following main result:

$$
N_{141}=\frac{2 T_{0}(4 c-3)}{6 c-3}- \begin{cases}4 T_{0}\left(\bar{N}_{141}\left(t_{3}\right)-\bar{N}_{141}\left(t_{4}\right)\right) & \text { for } v_{*}<\frac{1-2 c}{\sqrt{2}}, \\ 0 & \text { for } v_{*} \geq \frac{1-2 c}{\sqrt{2}} .\end{cases}
$$

Taking into account (2.225), integral $N_{142}$ is equal to

$$
N_{142}=N_{132}=\frac{2(4 c-3)}{6 c-3}\left(v_{*}-\beta v_{*}^{3}\right) .
$$


On the other hand, according to (2.227), integral $N_{133}$ is as follows:

$$
N_{143}=N_{133}=\frac{2}{3} \sqrt{2(1-2 c)}\left(3 \beta v_{*}^{2}-\alpha\right)
$$

Taking into account (2.228), integral $N_{144}$ is defined in the following way:

$$
\begin{aligned}
& N_{144}=\frac{12 \beta v_{*}}{360}(2 c-1)\left(4725-18216 c+17536 c^{2}+30 \sqrt{1-2 c}(189+8 c(64 c-79))\right) \\
& \times(30(2 c-1)(7695+4 c(-13587+8 c(4419+128 c(16 c-39)))) \\
&-4 \sqrt{1-2 c}(69255+c(-555705+8 c(209115+32 c(4392 c-8747))))+(8 c-3) \\
& \times\left(-1485+8394 c-15712 c^{2}+9728 c^{3}\right. \\
&\quad+\sqrt{1-2 c}(-1485+4 c(2751+16 c(320 c-411)))) \\
& \times F\left(1,1+\frac{1}{2 \sqrt{1-2 c}} ; 4+\frac{1}{2 \sqrt{1-2 c}} ;-1\right)+2(3-8 c)^{2}(8 c-5) \\
& \times(-12+24 c+\sqrt{1-2 c}(40 c-21)) \cdot F\left(1,2+\frac{1}{2 \sqrt{1-2 c}} ; 5+\frac{1}{2 \sqrt{1-2 c}} ;-1\right) \\
&+(8 c-3)\left(45+102 c-896 c^{2}+1024 c^{3}+\sqrt{1-2 c}(-45+4 c(207+16 c(32 c-39)))\right) \\
&\left.\times F\left(1,3+\frac{1}{2 \sqrt{1-2 c}} ; 6+\frac{1}{2 \sqrt{1-2 c}} ;-1\right)\right) .
\end{aligned}
$$

According to (2.229), integral $N_{145}$ is

$$
\begin{aligned}
& N_{145}=\frac{1024 \sqrt{2}(1-2 c)^{3} \beta}{(1+3 \sqrt{1-2 c})(1+55 \sqrt{1-2 c})(1+7 \sqrt{1-2 c})(1+9 \sqrt{1-2 c})(1+11 \sqrt{1-2 c})(1+13 \sqrt{1-2 c})} \\
& \times\left(\left(252(1+\sqrt{1-2 c})-3(327+263 \sqrt{1-2 c}) c+(954+572 \sqrt{1-2 c}) c^{2}\right)\right. \\
& \times F\left(8, \frac{1}{2}\left(3+\frac{1}{2 \sqrt{1-2 c}}\right) ; \frac{1}{2}\left(11+\frac{1}{2 \sqrt{1-2 c}}\right) ;-1\right) \\
&-2(8 c-3)(-8-20 \sqrt{1-2 c}+16 c+39 c \sqrt{1-2 c}) \\
& \cdot F\left(8, \frac{1}{2}\left(5+\frac{1}{2 \sqrt{1-2 c}}\right) ; \frac{1}{2}\left(13+\frac{1}{2 \sqrt{1-2 c}}\right) ;-1\right) \\
&+\left(12(\sqrt{1-2 c}-1)-53 c(\sqrt{1-2 c}-1)+c^{2}(60 \sqrt{1-2 c}-58)\right) \\
&\left.\times F\left(8, \frac{1}{2}\left(7+\frac{1}{2 \sqrt{1-2 c}}\right) ; \frac{1}{2}\left(15+\frac{1}{2 \sqrt{1-2 c}}\right) ;-1\right)\right) .
\end{aligned}
$$


Substituting the above formulas into (2.231), one obtains

$$
\begin{aligned}
N_{14}= & \frac{2(4 c-3)}{6 c-3}\left(v_{*}-\beta v_{*}^{3}+T_{0}\right)+\frac{2}{3} \sqrt{2(1-2 c)}\left(3 \beta v_{*}^{2}-\alpha\right)-N_{144}+N_{145} \\
& - \begin{cases}4 T_{0}\left(\bar{N}_{141}\left(t_{3}\right)-\bar{N}_{141}\left(t_{4}\right)\right) & \text { for } v_{*}<\frac{1-2 c}{\sqrt{2}}, \\
0 & \text { for } v_{*} \geq \frac{1-2 c}{\sqrt{2}} .\end{cases}
\end{aligned}
$$

Substitution of the above formulas into (2.207) gives

$$
\begin{aligned}
N_{1}\left(t_{0}\right)= & \tilde{A} \sin \left(\omega t_{0}-\kappa\right)-\frac{2 \sqrt{2(1-2 c)}}{3}\left(\delta_{11}-\delta_{21}\right)+N_{123}+N_{124} \\
& -\frac{\sqrt{2}}{3(2 c-1)}\left(\alpha_{21}-\alpha_{11}\right)\left(3 \sqrt{1-2 c}+c \psi\left(\frac{1}{4}\left(1+\frac{1}{\sqrt{1-2 c}}\right)\right)-c \psi\left(\frac{1}{4}\left(3+\frac{1}{\sqrt{1-2 c}}\right)\right)\right) \\
& +\frac{4}{3} \sqrt{2(1-2 c)}\left(3 \beta v_{*}^{2}-\alpha\right)+N_{134}+N_{135}-N_{144}+N_{145} \\
& + \begin{cases}4 T_{0}\left(-\bar{N}_{141}\left(t_{3}\right)+\bar{N}_{141}\left(t_{4}\right)\right) & \text { for } v_{*}<\sqrt{\frac{1-2 c}{2}}, \\
0 & \text { for } v_{*} \geq \sqrt{\frac{1-2 c}{2}} .\end{cases}
\end{aligned}
$$

Finally, the next criterion of chaos is defined in the following way:

$$
\begin{aligned}
\tilde{A}>\mid & -\frac{2 \sqrt{2(1-2 c)}}{3}\left(\delta_{11}-\delta_{21}\right)+N_{123}+N_{124} \\
& -\frac{\sqrt{2}}{3(2 c-1)}\left(\alpha_{21}-\alpha_{11}\right)\left(3 \sqrt{1-2 c}+c \psi\left(\frac{1}{4}\left(1+\frac{1}{\sqrt{1-2 c}}\right)\right)-c \psi\left(\frac{1}{4}\left(3+\frac{1}{\sqrt{1-2 c}}\right)\right)\right) \\
& +\frac{4}{3} \sqrt{2(1-2 c)}\left(3 \beta v_{*}^{2}-\alpha\right)+N_{134}+N_{135}-N_{144}+N_{145} \\
+ & \begin{cases}4 T_{0}\left(-\bar{N}_{141}\left(t_{3}\right)+\bar{N}_{141}\left(t_{4}\right)\right) & \text { for } v_{*}<\sqrt{\frac{1-2 c}{2}} \\
0 & \text { for } v_{*} \geq \sqrt{\frac{1-2 c}{2}}\end{cases}
\end{aligned}
$$

According to (2.84), the Melnikov function is

$$
N_{2}\left(t_{0}\right)=N_{21}\left(t_{0}\right)-N_{22}-N_{23}-N_{24},
$$


where

$$
\begin{aligned}
N_{21}\left(t_{0}\right) & =2 A \gamma \int_{-\infty}^{\infty} \dot{r}(\sqrt{1-2 c} t) \cos \omega\left(t+t_{0}\right) d t \\
& =-2 A \gamma \sqrt{1-2 c} \int_{-\infty}^{\infty} \operatorname{sech}(\sqrt{1-2 c} t) \operatorname{tgh}(\sqrt{1-2 c} t) \cos \omega\left(t+t_{0}\right) d t \\
N_{22} & =2 A \int_{-\infty}^{\infty}\left(\delta_{1}\left(x_{10}, \dot{x}_{10}\right)-\delta_{2}\left(x_{10}, \dot{x}_{10}\right)\right) \dot{r}(\sqrt{1-2 c} t) d t \\
N_{23} & =2 A \int_{-\infty}^{\infty} \dot{r}(\sqrt{1-2 c} t) T\left(\dot{x}_{01}-v_{*}\right) d t \\
& =-2 A \sqrt{1-2 c} \int_{-\infty}^{\infty} \operatorname{sech}(\sqrt{1-2 c} t) \operatorname{tgh}(\sqrt{1-2 c} t) T\left(\dot{x}_{01}-v_{*}\right) d t \\
N_{24} & =2 A \int_{-\infty}^{\infty} \dot{r}(\sqrt{1-2 c} t) T\left(-\dot{x}_{01}-v_{*}\right) d t \\
& =-2 A \sqrt{1-2 c} \int_{-\infty}^{\infty} \operatorname{sech}(\sqrt{1-2 c} t) \operatorname{tgh}(\sqrt{1-2 c} t) T\left(-\dot{x}_{01}-v_{*}\right) d t .
\end{aligned}
$$

The formula for $N_{21}\left(t_{0}\right)$ is as follows:

$$
N_{21}\left(t_{0}\right)=N_{211}\left(t_{0}\right)-N_{212}\left(t_{0}\right)
$$

where

$$
\begin{aligned}
& N_{211}\left(t_{0}\right)=-2 A \gamma \sqrt{1-2 c} \cos \omega t_{0} \int_{-\infty}^{\infty} \operatorname{sech}(\sqrt{1-2 c} t) \operatorname{tgh}(\sqrt{1-2 c} t) \cos \omega t d t, \\
& N_{212}\left(t_{0}\right)=-2 A \gamma \sqrt{1-2 c} \sin \omega t_{0} \int_{-\infty}^{\infty} \operatorname{sech}(\sqrt{1-2 c} t) \operatorname{tgh}(\sqrt{1-2 c} t) \sin \omega t d t .
\end{aligned}
$$

Upon integration we have

$$
\begin{gathered}
N_{211}\left(t_{0}\right)=0 \\
N_{212}\left(t_{0}\right)=-\frac{2 \pi A \gamma \omega}{\sqrt{1-2 c}} \operatorname{sech}\left(\frac{\pi \omega}{2 \sqrt{1-2 c}}\right) \sin \omega t_{0} .
\end{gathered}
$$

Substitution of the above formulas into (2.247) gives

$$
N_{21}\left(t_{0}\right)=\frac{2 \pi A \gamma \omega}{\sqrt{1-2 c}} \operatorname{sech}\left(\frac{\pi \omega}{2 \sqrt{1-2 c}}\right) \sin \omega t_{0} .
$$

Formula $N_{22}$ is defined by

$$
N_{22}=-N_{221}+N_{222}+N_{223}+N_{224} \text {, }
$$


where

$$
\begin{aligned}
N_{221}= & 2 \sqrt{2(1-2 c)} A\left(\alpha_{11}-\alpha_{21}\right) \int_{-\infty}^{\infty} \operatorname{sech}^{2}(\sqrt{1-2 c} t) \operatorname{tgh}(\sqrt{1-2 c} t) d t \\
N_{222}= & 2 \sqrt{2}(1-2 c) A\left(\delta_{11}-\delta_{21}\right) \int_{-\infty}^{\infty} \operatorname{sech}^{2}(\sqrt{1-2 c} t) \operatorname{tgh}^{2}(\sqrt{1-2 c} t) d t \\
N_{223}= & 4(1-2 c)^{2} A\left(\delta_{12}-\delta_{22}\right) \\
& \times \int_{-\infty}^{\infty} \operatorname{sech}^{3}(\sqrt{1-2 c} t) \operatorname{tgh}^{3}(\sqrt{1-2 c} t) \operatorname{sgn}(\operatorname{sech}(\sqrt{1-2 c} t) \operatorname{tgh}(\sqrt{1-2 c} t)) d t \\
= & 8(1-2 c)^{2} A\left(\delta_{12}-\delta_{22}\right) \int_{0}^{\infty} \operatorname{sech}^{3}(\sqrt{1-2 c} t) \operatorname{tgh}^{3}(\sqrt{1-2 c} t) d t \\
N_{224}= & 4 \sqrt{2}(1-2 c)^{3} A\left(\delta_{13}-\delta_{23}\right) \int_{-\infty}^{\infty} \operatorname{sech}^{4}(\sqrt{1-2 c} t) \operatorname{tgh}^{4}(\sqrt{1-2 c} t) d t .
\end{aligned}
$$

Carrying out the computations, one gets

$$
\begin{gathered}
N_{221}=0, \quad N_{222}=\frac{4 A \sqrt{2(1-2 c)}}{3}\left(\delta_{11}-\delta_{21}\right), \\
N_{223}=\frac{16 A(1-2 c)^{3 / 2}}{15}\left(\delta_{12}-\delta_{22}\right), \quad N_{224}=\frac{16 \sqrt{2} A(1-2 c)^{5 / 2}}{15}\left(\delta_{13}-\delta_{23}\right) .
\end{gathered}
$$

Substitution of the above formulas into (2.251) gives

$$
N_{22}=\frac{4 A}{3}\left(\sqrt{2(1-2 c)}\left(\delta_{11}-\delta_{21}\right)+\frac{4}{5}(1-2 c)^{3 / 2}\left(\delta_{12}-\delta_{22}\right)+\frac{4 \sqrt{2}}{5}(1-2 c)^{5 / 2}\left(\delta_{13}-\delta_{23}\right)\right)
$$

According to (2.5), the formula for $N_{23}$ takes the form

$$
N_{23}=-N_{231}-N_{232}+N_{233}+N_{234}+N_{235}
$$

where

$$
\begin{aligned}
& N_{231}=2 A T_{0} \sqrt{1-2 c} \int_{-\infty}^{\infty} \operatorname{sech}(\sqrt{1-2 c} t) \operatorname{tgh}(\sqrt{1-2 c} t) \operatorname{sgn}\left(\dot{x}_{01}-v_{*}\right) d t \\
& N_{232}=2 A\left(v_{*}-\beta v_{*}^{3}\right) \sqrt{1-2 c} \int_{-\infty}^{\infty} \operatorname{sech}(\sqrt{1-2 c} t) \operatorname{tgh}(\sqrt{1-2 c} t) d t \\
& N_{233}=2 \sqrt{2} A\left(3 \beta v_{*}^{2}-\alpha\right)(1-2 c)^{3 / 2} \int_{-\infty}^{\infty} \operatorname{sech}^{2}(\sqrt{1-2 c} t) \operatorname{tgh}^{2}(\sqrt{1-2 c} t) d t \\
& N_{234}=12 A \beta v_{*}(1-2 c)^{5 / 2} \int_{-\infty}^{\infty} \operatorname{sech}^{3}(\sqrt{1-2 c} t) \operatorname{tgh}^{3}(\sqrt{1-2 c} t) d t \\
& N_{235}=4 \sqrt{2} A \beta(1-2 c)^{7 / 2} \int_{-\infty}^{\infty} \operatorname{sech}^{4}(\sqrt{1-2 c} t) \operatorname{tgh}^{4}(\sqrt{1-2 c} t) d t
\end{aligned}
$$


Integral $N_{231}$ for $\left|v_{*}\right|>(1-2 c) / \sqrt{2}$ is defined in the following way:

$$
N_{231}=-2 A T_{0} \sqrt{1-2 c} \int_{-\infty}^{\infty} \operatorname{sech}(\sqrt{1-2 c} t) \operatorname{tgh}(\sqrt{1-2 c} t) d t=0 .
$$

For $\left|v_{*}\right|<(1-2 c) / \sqrt{2}$, one has

$$
\begin{aligned}
N_{231}=2 A T_{0} \sqrt{1-2 c}( & -\int_{-\infty}^{t_{1}} \operatorname{sech}(\sqrt{1-2 c} t) \operatorname{tgh}(\sqrt{1-2 c} t) d t \\
& +\int_{t_{1}}^{t_{2}} \operatorname{sech}(\sqrt{1-2 c} t) \operatorname{tgh}(\sqrt{1-2 c} t) d t \\
& \left.-\int_{t_{2}}^{\infty} \operatorname{sech}(\sqrt{1-2 c} t) \operatorname{tgh}(\sqrt{1-2 c} t) d t\right) .
\end{aligned}
$$

Carrying out the integration, one gets

$$
\begin{aligned}
N_{231} & =2 A T_{0}\left(\left.\operatorname{sech}(\sqrt{1-2 c} t)\right|_{-\infty} ^{t_{1}}-\left.\operatorname{sech}(\sqrt{1-2 c} t)\right|_{t_{1}} ^{t_{2}}+\left.\operatorname{sech}(\sqrt{1-2 c} t)\right|_{t_{2}} ^{\infty}\right) \\
& =4 A T_{0}\left(\operatorname{sech}\left(\sqrt{1-2 c} t_{1}\right)-\operatorname{sech}\left(\sqrt{1-2 c} t_{2}\right)\right),
\end{aligned}
$$

and hence

$$
N_{231}=4 A T_{0}\left(\sqrt{\frac{1}{2}+\sqrt{\frac{1}{4}-\frac{v_{*}^{2}}{2(1-2 c)^{2}}}}-\sqrt{\frac{1}{2}-\sqrt{\frac{1}{4}-\frac{v_{*}^{2}}{2(1-2 c)^{2}}}}\right)
$$

The following main result of this part of consideration is reported:

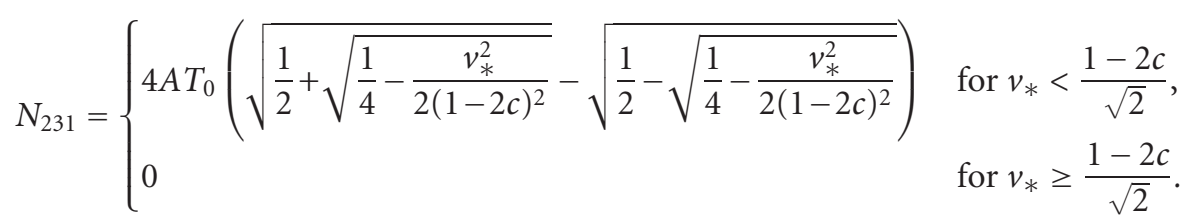

Observe that

$$
N_{232}=N_{234}=0
$$

On the other hand, integral $N_{233}$ is as follows:

$$
N_{233}=\left.\frac{2 \sqrt{2}}{3} A\left(3 \beta v_{*}^{2}-\alpha\right)(1-2 c) \operatorname{tgh}^{3}(\sqrt{1-2 c} t)\right|_{-\infty} ^{\infty}=\frac{4 \sqrt{2}}{3} A(1-2 c)\left(3 \beta v_{*}^{2}-\alpha\right) .
$$


54 Stick-slip chaos in a self-excited oscillator

Integral $N_{235}$ is equal to

$$
N_{235}=\left.\frac{4 \sqrt{2}}{35} A \beta(1-2 c)^{3}(6+\cosh (2 \sqrt{1-2 c} t)) \operatorname{sech}^{2}(\sqrt{1-2 c} t) \operatorname{tgh}^{5}(\sqrt{1-2 c} t)\right|_{-\infty} ^{\infty},
$$

and therefore

$$
N_{235}=\frac{16 \sqrt{2}}{35} A \beta(1-2 c)^{3}
$$

Substitution of the above formulas into (2.255) gives

$$
\begin{aligned}
N_{23}= & \frac{4 \sqrt{2}}{3} A\left(3 \beta v_{*}^{2}-\alpha\right)(1-2 c)+\frac{16 \sqrt{2}}{35} A \beta(1-2 c)^{3} \\
& - \begin{cases}4 A T_{0}\left(\sqrt{\frac{1}{2}-\sqrt{\frac{1}{4}-\frac{v_{*}^{2}}{2(1-2 c)^{2}}}}-\sqrt{\frac{1}{2}+\sqrt{\frac{1}{4}-\frac{v_{*}^{2}}{2(1-2 c)^{2}}}}\right) & \text { for } v_{*}<\frac{1-2 c}{\sqrt{2}}, \\
0 & \text { for } v_{*} \geq \frac{1-2 c}{\sqrt{2}} .\end{cases}
\end{aligned}
$$

According to (2.5), $N_{24}$ takes the form

$$
N_{24}=-N_{241}-N_{242}-N_{243}+N_{244}-N_{245}
$$

where

$$
\begin{aligned}
& N_{241}=2 A T_{0} \sqrt{1-2 c} \int_{-\infty}^{\infty} \operatorname{sech}(\sqrt{1-2 c} t) \operatorname{tgh}(\sqrt{1-2 c} t) \operatorname{sgn}\left(-\dot{x}_{01}-v_{*}\right) d t, \\
& N_{242}=2 A\left(v_{*}-\beta v_{*}^{3}\right) \sqrt{1-2 c} \int_{-\infty}^{\infty} \operatorname{sech}(\sqrt{1-2 c} t) \operatorname{tgh}(\sqrt{1-2 c} t) d t \\
& N_{243}=2 \sqrt{2} A\left(3 \beta v_{*}^{2}-\alpha\right)(1-2 c)^{3 / 2} \int_{-\infty}^{\infty} \operatorname{sech}^{2}(\sqrt{1-2 c} t) \operatorname{tgh}^{2}(\sqrt{1-2 c} t) d t \\
& N_{244}=12 A \beta v_{*}(1-2 c)^{5 / 2} \int_{-\infty}^{\infty} \operatorname{sech}^{3}(\sqrt{1-2 c} t) \operatorname{tgh}^{3}(\sqrt{1-2 c} t) d t \\
& N_{245}=4 \sqrt{2} A \beta(1-2 c)^{7 / 2} \int_{-\infty}^{\infty} \operatorname{sech}^{4}(\sqrt{1-2 c} t) \operatorname{tgh}^{4}(\sqrt{1-2 c} t) d t
\end{aligned}
$$

Computing integral $N_{241}$ for $\left|v_{*}\right|>(1-2 c) / \sqrt{2}$, one gets

$$
N_{241}=-2 A T_{0} \sqrt{1-2 c} \int_{-\infty}^{\infty} \operatorname{sech}(\sqrt{1-2 c} t) \operatorname{tgh}(\sqrt{1-2 c} t) d t=0 .
$$


In the second case, that is, for $\left|v_{*}\right|<(1-2 c) / \sqrt{2}$, one obtains

$$
\begin{aligned}
N_{241}=2 A T_{0} \sqrt{1-2 c}( & -\int_{-\infty}^{t_{3}} \operatorname{sech}(\sqrt{1-2 c} t) \operatorname{tgh}(\sqrt{1-2 c} t) d t \\
& +\int_{t_{3}}^{t_{4}} \operatorname{sech}(\sqrt{1-2 c} t) \operatorname{tgh}(\sqrt{1-2 c} t) d t \\
& \left.-\int_{t_{4}}^{\infty} \operatorname{sech}(\sqrt{1-2 c} t) \operatorname{tgh}(\sqrt{1-2 c} t) d t\right),
\end{aligned}
$$

where $t_{3}, t_{4}$ are as follows:

$$
\begin{aligned}
& t_{3}=-t_{2}=\frac{1}{\sqrt{1-2 c}} \operatorname{arcsech} \sqrt{\frac{1}{2}-\sqrt{\frac{1}{4}-\frac{v_{*}^{2}}{2(1-2 c)^{2}}}}, \\
& t_{4}=-t_{1}=\frac{1}{\sqrt{1-2 c}} \operatorname{arcsech} \sqrt{\frac{1}{2}+\sqrt{\frac{1}{4}-\frac{v_{*}^{2}}{2(1-2 c)^{2}}}} .
\end{aligned}
$$

Upon integration one has

$$
\begin{aligned}
N_{241} & =2 A T_{0}\left(\left.\operatorname{sech}(\sqrt{1-2 c} t)\right|_{-\infty} ^{t_{3}}-\left.\operatorname{sech}(\sqrt{1-2 c} t)\right|_{t_{3}} ^{t_{4}}+\left.\operatorname{sech}(\sqrt{1-2 c} t)\right|_{t_{4}} ^{\infty}\right) \\
& =4 A T_{0}\left(\operatorname{sech}\left(\sqrt{1-2 c} t_{3}\right)-\operatorname{sech}\left(\sqrt{1-2 c} t_{4}\right)\right),
\end{aligned}
$$

and hence

$$
N_{241}=4 A T_{0}\left(\sqrt{\frac{1}{2}-\sqrt{\frac{1}{4}-\frac{v_{*}^{2}}{2(1-2 c)^{2}}}}-\sqrt{\frac{1}{2}+\sqrt{\frac{1}{4}-\frac{v_{*}^{2}}{2(1-2 c)^{2}}}}\right),
$$

and finally

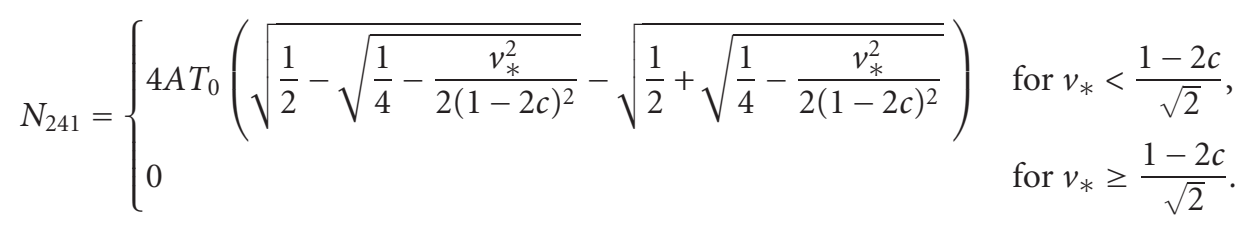

Since

$$
\begin{gathered}
N_{242}=N_{244}=0, \\
N_{243}=N_{233}, \\
N_{245}=N_{235},
\end{gathered}
$$


and according to (2.267) one obtains

$$
\begin{aligned}
N_{24}= & -\frac{4 \sqrt{2}}{3} A\left(3 \beta v_{*}^{2}-\alpha\right)(1-2 c)-\frac{16 \sqrt{2}}{35} A \beta(1-2 c)^{3} \\
& - \begin{cases}4 A T_{0}\left(\sqrt{\frac{1}{2}-\sqrt{\frac{1}{4}-\frac{v_{*}^{2}}{2(1-2 c)^{2}}}}-\sqrt{\frac{1}{2}+\sqrt{\frac{1}{4}-\frac{v_{*}^{2}}{2(1-2 c)^{2}}}}\right) & \text { for } v_{*}<\frac{1-2 c}{\sqrt{2}}, \\
0 & \text { for } v_{*} \geq \frac{1-2 c}{\sqrt{2}} .\end{cases}
\end{aligned}
$$

Substituting (2.250), (2.254), (2.266), and (2.276) into (2.245), the following Melnikov function is obtained:

$$
\begin{aligned}
N_{2}\left(t_{0}\right)= & \frac{2 \pi A \gamma \omega}{\sqrt{1-2 c}} \operatorname{sech}\left(\frac{\pi \omega}{2 \sqrt{1-2 c}}\right) \sin \omega t_{0} \\
& -\frac{4 A}{3}\left(\sqrt{2(1-2 c)}\left(\delta_{11}-\delta_{21}\right)+\frac{4}{5}(1-2 c)^{3 / 2}\left(\delta_{12}-\delta_{22}\right)+\frac{4 \sqrt{2}}{5}(1-2 c)^{5 / 2}\left(\delta_{13}-\delta_{23}\right)\right) \\
& + \begin{cases}8 A T_{0}\left(\sqrt{\frac{1}{2}-\sqrt{\frac{1}{4}-\frac{v_{*}^{2}}{2(1-2 c)^{2}}}}-\sqrt{\frac{1}{2}+\sqrt{\frac{1}{4}-\frac{v_{*}^{2}}{2(1-2 c)^{2}}}}\right) & \text { for } v_{*}<\frac{1-2 c}{\sqrt{2}} \\
0 & \text { for } v_{*} \geq \frac{1-2 c}{\sqrt{2}} .\end{cases}
\end{aligned}
$$

The condition of stable and unstable manifolds intersection is

$$
\begin{aligned}
& \frac{2 \pi|A| \gamma \omega}{\sqrt{1-2 c}} \operatorname{sech}\left(\frac{\pi \omega}{2 \sqrt{1-2 c}}\right) \\
& > \\
& >-\frac{4 A}{3}\left(\sqrt{2(1-2 c)}\left(\delta_{11}-\delta_{21}\right)+\frac{4}{5}(1-2 c)^{3 / 2}\left(\delta_{12}-\delta_{22}\right)+\frac{4 \sqrt{2}}{5}(1-2 c)^{5 / 2}\left(\delta_{13}-\delta_{23}\right)\right) \\
& +\left\{\begin{array}{ll}
8 A T_{0}\left(\sqrt{\frac{1}{2}-\sqrt{\frac{1}{4}-\frac{v_{*}^{2}}{2(1-2 c)^{2}}}}-\sqrt{\frac{1}{2}+\sqrt{\frac{1}{4}-\frac{v_{*}^{2}}{2(1-2 c)^{2}}}}\right) & \text { for } v_{*}<\frac{1-2 c}{\sqrt{2}} \\
0 & \text { for } v_{*} \geq \frac{1-2 c}{\sqrt{2}}
\end{array} .\right.
\end{aligned}
$$

According to (2.87), the Melnikov function is defined as follows:

$$
N_{3}\left(t_{0}\right)=-N_{31}\left(t_{0}\right)+N_{32}+N_{33}+N_{34}
$$


where

$$
\begin{aligned}
N_{31}\left(t_{0}\right) & =2 \gamma \int_{-\infty}^{0} R(\sqrt{1-2 c} t) e^{t} \cos \omega\left(t+t_{0}\right) d t \\
& =2 \gamma \int_{-\infty}^{0}\left(\frac{3-4 c}{3(1-2 c)}-\operatorname{sech}^{2}(\sqrt{1-2 c} t)-\frac{\operatorname{tgh}(\sqrt{1-2 c} t)}{\sqrt{1-2 c}}\right) e^{t} \cos \omega\left(t+t_{0}\right) d t, \\
N_{32} & =2 \int_{-\infty}^{0}\left(\delta_{1}\left(x_{10}, \dot{x}_{10}\right)-\delta_{2}\left(x_{10}, \dot{x}_{10}\right)\right) e^{t} R(\sqrt{1-2 c} t) d t \\
N_{33} & =2 \int_{-\infty}^{0} e^{t} R(\sqrt{1-2 c} t) T\left(\dot{x}_{01}-v_{*}\right) d t \\
& =2 \int_{-\infty}^{0} e^{t}\left(\frac{3-4 c}{3(1-2 c)}-\operatorname{sech}^{2}(\sqrt{1-2 c} t)-\frac{\operatorname{tgh}(\sqrt{1-2 c} t)}{\sqrt{1-2 c}}\right) T\left(\dot{x}_{01}-v_{*}\right) d t, \\
N_{34} & =2 \int_{-\infty}^{0} e^{t} R(\sqrt{1-2 c} t) T\left(-\dot{x}_{01}-v_{*}\right) d t \\
& =2 \int_{-\infty}^{0} e^{t}\left(\frac{3-4 c}{3(1-2 c)}-\operatorname{sech}^{2}(\sqrt{1-2 c} t)-\frac{\operatorname{tgh}(\sqrt{1-2 c} t)}{\sqrt{1-2 c}}\right) T\left(-\dot{x}_{01}-v_{*}\right) d t .
\end{aligned}
$$

Formula for $N_{31}\left(t_{0}\right)$ is as follows:

$$
N_{31}\left(t_{0}\right)=N_{311}\left(t_{0}\right)-N_{312}\left(t_{0}\right)
$$

where

$$
\begin{aligned}
& N_{311}\left(t_{0}\right)=2 \gamma \cos \omega t_{0} \int_{-\infty}^{0}\left(\frac{3-4 c}{3(1-2 c)}-\operatorname{sech}^{2}(\sqrt{1-2 c} t)-\frac{\operatorname{tgh}(\sqrt{1-2 c} t)}{\sqrt{1-2 c}}\right) e^{t} \cos \omega t d t \\
& N_{312}\left(t_{0}\right)=2 \gamma \sin \omega t_{0} \int_{-\infty}^{0}\left(\frac{3-4 c}{3(1-2 c)}-\operatorname{sech}^{2}(\sqrt{1-2 c} t)-\frac{\operatorname{tgh}(\sqrt{1-2 c} t)}{\sqrt{1-2 c}}\right) e^{t} \sin \omega t d t .
\end{aligned}
$$

Comparing with (2.210) and taking into account (2.213), one gets

$$
\begin{aligned}
N_{311}\left(t_{0}\right)=N_{111}\left(t_{0}\right)=\gamma \cos \omega t_{0}( & \frac{6 \sqrt{1-2 c} \omega^{2}-2(4 c-3)}{3(2 c-1)\left(1+\omega^{2}\right)} \\
& \left.+\frac{\omega}{2 c-1}\left(\operatorname{Im} \psi\left(\frac{1+\omega i}{4 \sqrt{1-2 c}}\right)-\operatorname{Im} \psi\left(\frac{1}{4}\left(2+\frac{1+\omega i}{\sqrt{1-2 c}}\right)\right)\right)\right) .
\end{aligned}
$$


Comparing with (2.211) and according to (2.214), one has

$$
\begin{aligned}
N_{312}\left(t_{0}\right)=-N_{112}\left(t_{0}\right)=-\gamma \sin \omega t_{0}( & -\frac{2 \omega((4 c-3)+3 \sqrt{1-2 c})}{3(2 c-1)\left(1+\omega^{2}\right)} \\
& \left.+\frac{\omega}{2 c-1}\left(\operatorname{Re} \psi\left(\frac{1+\omega i}{4 \sqrt{1-2 c}}\right)-\operatorname{Re} \psi\left(\frac{1}{4}\left(2+\frac{1-\omega i}{\sqrt{1-2 c}}\right)\right)\right)\right) .
\end{aligned}
$$

Substituting the above formulas into (2.281), one obtains

$$
N_{31}\left(t_{0}\right)=\gamma\left(A_{1} \cos \omega t_{0}+A_{2} \sin \omega t_{0}\right)=\tilde{A} \sin \left(\omega t_{0}+\kappa\right),
$$

where

$$
\begin{aligned}
& A_{1}=\frac{6 \sqrt{1-2 c} \omega^{2}-2(4 c-3)}{3(2 c-1)\left(1+\omega^{2}\right)}+\frac{\omega}{2 c-1}\left(\operatorname{Im} \psi\left(\frac{1+\omega i}{4 \sqrt{1-2 c}}\right)-\operatorname{Im} \psi\left(\frac{1}{4}\left(2+\frac{1+\omega i}{\sqrt{1-2 c}}\right)\right)\right), \\
& A_{2}=-\frac{2 \omega((4 c-3)+3 \sqrt{1-2 c})}{3(2 c-1)\left(1+\omega^{2}\right)}+\frac{\omega}{2 c-1}\left(\operatorname{Re} \psi\left(\frac{1+\omega i}{4 \sqrt{1-2 c}}\right)-\operatorname{Re} \psi\left(\frac{1}{4}\left(2+\frac{1-\omega i}{\sqrt{1-2 c}}\right)\right)\right),
\end{aligned}
$$

and $\tilde{A}=\gamma \sqrt{A_{1}^{2}+A_{2}^{2}}, \operatorname{tg} \kappa=A_{1} / A_{2}$.

The formula for $N_{32}$ is as follows:

$$
N_{32}=N_{321}-N_{322}-N_{323}-N_{324},
$$

where

$$
\begin{aligned}
N_{321} & =2 \sqrt{2(1-2 c)}\left(\alpha_{11}-\alpha_{21}\right) \int_{-\infty}^{0} \operatorname{sech}(\sqrt{1-2 c} t) e^{t} R(\sqrt{1-2 c} t) d t \\
N_{322} & =2 \sqrt{2}(1-2 c)\left(\delta_{11}-\delta_{21}\right) \int_{-\infty}^{0} \operatorname{sech}(\sqrt{1-2 c} t) \operatorname{tgh}(\sqrt{1-2 c} t) e^{t} R(\sqrt{1-2 c} t) d t, \\
N_{323} & =4(1-2 c)^{2}\left(\delta_{12}-\delta_{22}\right) \int_{-\infty}^{0} \operatorname{sech}^{2}(\sqrt{1-2 c} t) \operatorname{tgh}^{2}(\sqrt{1-2 c} t) \\
& =-4(1-2 c)^{2}\left(\delta_{12}-\delta_{22}\right) \int_{-\infty}^{0} \operatorname{sech}^{2}(\sqrt{1-2 c} t) \operatorname{tgh}^{2}(\sqrt{1-2 c} t) e^{t} R(\sqrt{1-2 c} t) d t, \\
N_{324} & =4 \sqrt{2}(1-2 c)^{3}\left(\delta_{13}-\delta_{23}\right) \int_{-\infty}^{0} \operatorname{sech}^{3}(\sqrt{1-2 c} t) \operatorname{tgh}^{3}(\sqrt{1-2 c} t) e^{t} R(\sqrt{1-2 c} t) d t .
\end{aligned}
$$




\section{Computations yield}

$$
\begin{aligned}
& N_{321}=\frac{\sqrt{2}}{3(1-2 c)}\left(\alpha_{11}-\alpha_{21}\right)\left(3 \sqrt{1-2 c}+c \psi\left(\frac{1}{4}\left(1+\frac{1}{\sqrt{1-2 c}}\right)\right)-c \psi\left(\frac{1}{4}\left(3+\frac{1}{\sqrt{1-2 c}}\right)\right)\right), \\
& N_{322}=-\frac{2}{3} \sqrt{2(1-2 c)}\left(\delta_{11}-\delta_{21}\right) \text {, } \\
& N_{323}=\frac{128(1-2 c)^{3 / 2}\left(\delta_{22}-\delta_{12}\right)}{3(1+2 \sqrt{1-2 c})(1+4 \sqrt{1-2 c})(1+6 \sqrt{1-2 c})(1+8 \sqrt{1-2 c})(1+10 \sqrt{1-2 c})} \\
& \times\left(\left(-6 a(191+152 \sqrt{1-2 c})+297(1+\sqrt{1-2 c})+16(69+40 \sqrt{1-2 c}) c^{2}\right)\right. \\
& \times F\left(6, \frac{1}{2}\left(2+\frac{1}{\sqrt{1-2 c}}\right) ; \frac{1}{2}\left(8+\frac{1}{\sqrt{1-2 c}}\right) ;-1\right) \\
& +(3+8 c)((-6(4+7 \sqrt{1-2 c})+16 c(3+5 \sqrt{1-2 c})) \\
& \times F\left(6, \frac{1}{2}\left(4+\frac{1}{\sqrt{1-2 c}}\right) ; \frac{1}{2}\left(10+\frac{1}{\sqrt{1-2 c}}\right) ;-1\right) \\
& +(-3+3 \sqrt{1-2 c}+6 c-8 c \sqrt{1-2 c}) \\
& \left.\left.F\left(6, \frac{1}{2}\left(6+\frac{1}{\sqrt{1-2 c}}\right) ; \frac{1}{2}\left(12+\frac{1}{\sqrt{1-2 c}}\right) ;-1\right)\right)\right) \\
& N_{324}=-\frac{1024 \sqrt{2}(1-2 c)^{3}\left(\delta_{23}-\delta_{13}\right)}{(1+3 \sqrt{1-2 c})(1+5 \sqrt{1-2 c})(1+7 \sqrt{1-2 c})(1+9 \sqrt{1-2 c})(1+11 \sqrt{1-2 c})(1+13 \sqrt{1-2 c})} \\
& \times\left(\left(252(1+\sqrt{1-2 c})-3 c(327+263 \sqrt{1-2 c})+(954+572 \sqrt{1-2 c}) c^{2}\right)\right. \\
& \times F\left(8, \frac{1}{2}\left(3+\frac{1}{\sqrt{1-2 c}}\right) ; \frac{1}{2}\left(11+\frac{1}{\sqrt{1-2 c}}\right) ;-1\right) \\
& +2(3+8 c)(-8-20 \sqrt{1-2 c}+16 c+39 c \sqrt{1-2 c}) \\
& F\left(8, \frac{1}{2}\left(5+\frac{1}{\sqrt{1-2 c}}\right) ; \frac{1}{2}\left(13+\frac{1}{\sqrt{1-2 c}}\right) ;-1\right) \\
& +\left(12(-1+\sqrt{1-2 c})-53 c(-1+\sqrt{1-2 c})+(-58+60 \sqrt{1-2 c}) c^{2}\right) \\
& \left.\times F\left(8, \frac{1}{2}\left(7+\frac{1}{\sqrt{1-2 c}}\right) ; \frac{1}{2}\left(15+\frac{1}{\sqrt{1-2 c}}\right) ;-1\right)\right) .
\end{aligned}
$$

Substitution of the above formulas into (2.287) gives

$$
\begin{aligned}
N_{32}= & \frac{\sqrt{2}}{3(1-2 c)}\left(\alpha_{11}-\alpha_{21}\right)\left(3 \sqrt{1-2 c}+c \psi\left(\frac{1}{4}\left(1+\frac{1}{\sqrt{1-2 c}}\right)\right)-c \psi\left(\frac{1}{4}\left(3+\frac{1}{\sqrt{1-2 c}}\right)\right)\right) \\
& +\frac{2}{3} \sqrt{2(1-2 c)}\left(\delta_{11}-\delta_{21}\right)-N_{323}-N_{324} .
\end{aligned}
$$


According to (2.5), one obtains

$$
N_{33}=N_{331}+N_{332}-N_{333}-N_{334}-N_{335},
$$

where

$$
\begin{aligned}
& N_{331}= 2 T_{0} \int_{-\infty}^{0} e^{t}\left(\frac{3-4 c}{3(1-2 c)}-\operatorname{sech}^{2}(\sqrt{1-2 c} t)-\frac{\operatorname{tgh}(\sqrt{1-2 c} t)}{\sqrt{1-2 c}}\right) \operatorname{sgn}\left(\dot{x}_{01}-v_{*}\right) d t, \\
& N_{332}= 2\left(v_{*}-\beta v_{*}^{3}\right) \int_{-\infty}^{0} e^{t}\left(\frac{3-4 c}{3(1-2 c)}-\operatorname{sech}^{2}(\sqrt{1-2 c} t)-\frac{\operatorname{tgh}(\sqrt{1-2 c} t)}{\sqrt{1-2 c}}\right) d t, \\
& N_{333}= 2 \sqrt{2}(1-2 c)\left(3 \beta v_{*}^{2}-\alpha\right) \\
& \times \int_{-\infty}^{0} e^{t}\left(\frac{3-4 c}{3(1-2 c)}-\operatorname{sech}^{2}(\sqrt{1-2 c} t)-\frac{\operatorname{tgh}(\sqrt{1-2 c} t)}{\sqrt{1-2 c}}\right) \\
& \operatorname{sech}(\sqrt{1-2 c t}) \operatorname{tgh}(\sqrt{1-2 c} t) d t, \\
& N_{334}= 12(1-2 c)^{2} \beta v_{*} \\
& \times \int_{-\infty}^{0} e^{t}\left(\frac{3-4 c}{3(1-2 c)}-\operatorname{sech}^{2}(\sqrt{1-2 c} t)-\frac{\operatorname{tgh}(\sqrt{1-2 c} t)}{\sqrt{1-2 c}}\right) \\
& \operatorname{sech}^{2}(\sqrt{1-2 c t}) \operatorname{tgh}^{2}(\sqrt{1-2 c} t) d t, \\
& N_{335}= 4 \sqrt{2}(1-2 c)^{3} \beta \\
& \times \int_{-\infty}^{0} e^{t}\left(\frac{3-4 c}{3(1-2 c)}-\operatorname{sech}^{2}(\sqrt{1-2 c} t)-\frac{\operatorname{tgh}(\sqrt{1-2 c} t)}{\sqrt{1-2 c}}\right) \\
& \operatorname{sech}^{3}(\sqrt{1-2 c t}) \operatorname{tgh}^{3}(\sqrt{1-2 c} t) d t .
\end{aligned}
$$

The formula $N_{331}$ for $\left|v_{*}\right|>(1-2 c) / \sqrt{2}$ is

$$
\begin{aligned}
N_{331} & =-2 T_{0} \int_{-\infty}^{0} e^{t}\left(\frac{3-4 c}{3(1-2 c)}-\operatorname{sech}^{2}(\sqrt{1-2 c} t)-\frac{\operatorname{tgh}(\sqrt{1-2 c} t)}{\sqrt{1-2 c}}\right) d t \\
& =-\bar{N}_{331}(0)+\lim _{t \rightarrow-\infty} \bar{N}_{331}(t)=-\frac{2 T_{0}(4 c-3)}{6 c-3},
\end{aligned}
$$

where

$$
\bar{N}_{331}(t)=\frac{2 T_{0} e^{t}(-3+4 c+3 \sqrt{1-2 c} \operatorname{tgh}(\sqrt{1-2 c} t))}{3(2 c-1)} .
$$


In the second case, that is, for $\left|v_{*}\right|<(1-2 c) / \sqrt{2}$, one gets

$$
\begin{aligned}
N_{331} & =2 T_{0}\left(-\left.\bar{N}_{331}(t)\right|_{-\infty} ^{t_{1}}+\left.\bar{N}_{331}(t)\right|_{t_{1}} ^{t_{2}}-\left.\bar{N}_{331}(t)\right|_{t_{2}} ^{0}\right) \\
& =-2 T_{0}\left(2\left(\bar{N}_{331}\left(t_{1}\right)-\bar{N}_{331}\left(t_{2}\right)\right)+\bar{N}_{331}(0)-\lim _{t \rightarrow-\infty} \bar{N}_{331}(t)\right) .
\end{aligned}
$$

The main result of these considerations is as follows:

$$
N_{331}=-\frac{2 T_{0}(4 c-3)}{6 c-3}- \begin{cases}4 T_{0}\left(\bar{N}_{331}\left(t_{1}\right)-\bar{N}_{331}\left(t_{2}\right)\right) & \text { for } v_{*}<\frac{1-2 c}{\sqrt{2}} \\ 0 & \text { for } v_{*} \geq \frac{1-2 c}{\sqrt{2}}\end{cases}
$$

Integral $N_{332}$ is

$$
\begin{aligned}
N_{332} & =2\left(v_{*}-\beta v_{*}^{3}\right) \int_{-\infty}^{0} e^{t}\left(\frac{3-4 c}{3(1-2 c)}-\operatorname{sech}^{2}(\sqrt{1-2 c} t)-\frac{\operatorname{tgh}(\sqrt{1-2 c} t)}{\sqrt{1-2 c}}\right) d t \\
& =\frac{2\left(v_{*}-\beta v_{*}^{3}\right)(4 c-3)}{6 c-3} .
\end{aligned}
$$

On the other hand, integral $N_{333}$ is equal to

$N_{333}$

$$
=\left.\frac{\sqrt{2}(1-2 c)\left(3 \beta v_{*}^{2}-\alpha\right) e^{t} \operatorname{sech}^{3}(\sqrt{1-2 c} t)(2 \sqrt{1-2 c} t \cosh (2 \sqrt{1-2 c} t)-\sinh (2 \sqrt{1-2 c} t))}{6 c-3}\right|_{-\infty} ^{0},
$$

and hence

$$
N_{333}=-\frac{2}{3} \sqrt{2(1-2 c)}\left(3 \beta v_{*}^{2}-\alpha\right)
$$

Integral $N_{334}$ is defined in the following way:

$$
\begin{aligned}
N_{334}= & \frac{384(1-2 c)^{2} \beta v_{*}}{3 \sqrt{1-2 c}(1+2 \sqrt{1-2 c})(1+4 \sqrt{1-2 c})(1+6 \sqrt{1-2 c})(1+8 \sqrt{1-2 c})(1+10 \sqrt{1-2 c})} \\
& \times\left(\left(297(1+\sqrt{1-2 c})-6(191+152 \sqrt{1-2 c}) c+16(69+40 \sqrt{1-2 c}) c^{2}\right)\right. \\
& \times F\left(6,1+\frac{1}{2 \sqrt{1-2 c}} ; 4+\frac{1}{2 \sqrt{1-2 c}} ;-1\right)
\end{aligned}
$$




$$
\begin{aligned}
& -(8 c-3)(-6(4+7 \sqrt{1-2 c})+80 c(1+\sqrt{1-2 c})) \\
& \cdot F\left(6,2+\frac{1}{2 \sqrt{1-2 c}} ; 5+\frac{1}{2 \sqrt{1-2 c}} ;-1\right) \\
& \left.+(3(\sqrt{1-2 c}-1)-2 c(4 \sqrt{1-2 c}-3)) F\left(6,3+\frac{1}{2 \sqrt{1-2 c}} ; 6+\frac{1}{2 \sqrt{1-2 c}} ;-1\right)\right) .
\end{aligned}
$$

Integral $N_{335}$ is equal to

$$
\begin{aligned}
N_{335}= & -\frac{1024 \sqrt{2}(1-2 c)^{3} \beta}{(1+3 \sqrt{1-2 c})(1+55 \sqrt{1-2 c})(1+7 \sqrt{1-2 c})(1+9 \sqrt{1-2 c})(1+11 \sqrt{1-2 c})(1+13 \sqrt{1-2 c})} \\
\times( & \left(252(1+\sqrt{1-2 c})-3(327+263 \sqrt{1-2 c}) c+(954+572 \sqrt{1-2 c}) c^{2}\right) \\
& \times F\left(8, \frac{1}{2}\left(3+\frac{1}{\sqrt{1-2 c}}\right) ; \frac{1}{2}\left(11+\frac{1}{\sqrt{1-2 c}}\right) ;-1\right) \\
& -2(8 c-3)(-8-20 \sqrt{1-2 c}+16 c+39 c \sqrt{1-2 c}) \\
& \cdot F\left(8, \frac{1}{2}\left(5+\frac{1}{\sqrt{1-2 c}}\right) ; \frac{1}{2}\left(13+\frac{1}{\sqrt{1-2 c}}\right) ;-1\right) \\
& +\left(12(\sqrt{1-2 c}-1)-53 c(\sqrt{1-2 c}-1)+c^{2}(60 \sqrt{1-2 c}-58)\right) \\
& \left.\times F\left(8, \frac{1}{2}\left(7+\frac{1}{\sqrt{1-2 c}}\right) ; \frac{1}{2}\left(15+\frac{1}{\sqrt{1-2 c}}\right) ;-1\right)\right) .
\end{aligned}
$$

Substitution of the above formulas into (2.291) gives

$$
\begin{aligned}
N_{33}= & \frac{2}{3} \sqrt{2(1-2 c)}\left(3 \beta v_{*}^{2}-\alpha\right)+\frac{2(4 c-3)}{6 c-3}\left(v_{*}-\beta v_{*}^{3}-T_{0}\right)-N_{334}-N_{335} \\
& - \begin{cases}4 T_{0}\left(\bar{N}_{331}\left(t_{1}\right)-\bar{N}_{331}\left(t_{2}\right)\right) & \text { for } v_{*}<\frac{1-2 c}{\sqrt{2}}, \\
0 & \text { for } v_{*} \geq \frac{1-2 c}{\sqrt{2}} .\end{cases}
\end{aligned}
$$

Applying (2.5), the formula for $N_{34}$ takes the form

$$
N_{34}=N_{341}+N_{342}+N_{343}-N_{344}+N_{345},
$$


where

$$
\begin{aligned}
& N_{341}= 2 T_{0} \int_{-\infty}^{0} e^{t}\left(\frac{3-4 c}{3(1-2 c)}-\operatorname{sech}^{2}(\sqrt{1-2 c} t)-\frac{\operatorname{tgh}(\sqrt{1-2 c} t)}{\sqrt{1-2 c}}\right) \operatorname{sgn}\left(-\dot{x}_{01}-v_{*}\right) d t, \\
& N_{342}= 2\left(v_{*}-\beta v_{*}^{3}\right) \int_{-\infty}^{0} e^{t}\left(\frac{3-4 c}{3(1-2 c)}-\operatorname{sech}^{2}(\sqrt{1-2 c} t)-\frac{\operatorname{tgh}(\sqrt{1-2 c} t)}{\sqrt{1-2 c}}\right) d t, \\
& N_{343}= 2 \sqrt{2}(1-2 c)\left(3 \beta v_{*}^{2}-\alpha\right) \\
& \times \int_{-\infty}^{0} e^{t}\left(\frac{3-4 c}{3(1-2 c)}-\operatorname{sech}^{2}(\sqrt{1-2 c} t)-\frac{\operatorname{tgh}(\sqrt{1-2 c} t)}{\sqrt{1-2 c}}\right) \\
& N_{344}= 12(1-2 c)^{2} \beta v_{*}(\sqrt{1-2 c} t) \operatorname{tgh}^{2}(\sqrt{1-2 c} t) d t, \\
& \times \int_{-\infty}^{0} e^{t}\left(\frac{3-4 c}{3(1-2 c)}-\operatorname{sech}^{2}(\sqrt{1-2 c} t)-\frac{\operatorname{tgh}(\sqrt{1-2 c} t)}{\sqrt{1-2 c}}\right) \\
& N_{345}= 4 \sqrt{2}(1-2 c)^{3} \beta \\
& \times \int_{-\infty}^{0} e^{t}\left(\frac{3-4 c}{3(1-2 c)}-\operatorname{sech}^{2}(\sqrt{1-2 c} t)-\frac{\operatorname{tgh}(\sqrt{1-2 c} t)}{\sqrt{1-2 c}}\right) \\
& \operatorname{sech}^{3}(\sqrt{1-2 c} t) \operatorname{tgh}^{3}(\sqrt{1-2 c} t) d t . \\
& \operatorname{sech}^{2}(\sqrt{1-2 c} t) d t, \\
& \operatorname{tgh}^{2}(\sqrt{1-2.304)})
\end{aligned}
$$

For $t_{3}>0$ and $t_{4}>0$, integral $N_{141}$ is defined as follows:

$$
N_{341}=N_{331}=-\bar{N}_{331}(0)+\lim _{t \rightarrow-\infty} \bar{N}_{331}(t)=-\frac{2 T_{0}(4 c-3)}{6 c-3} .
$$

According to (2.297), integral $N_{342}$ is

$$
N_{342}=N_{332}=\frac{2\left(v_{*}-\beta v_{*}^{3}\right)(4 c-3)}{6 c-3} .
$$

On the other hand, according to (2.299), the integral for $N_{343}$ is

$$
N_{343}=N_{333}=-\frac{2}{3} \sqrt{2(1-2 c)}\left(3 \beta v_{*}^{2}-\alpha\right)
$$


64 Stick-slip chaos in a self-excited oscillator

Taking into account (2.300), one obtains

$$
\begin{aligned}
N_{344}= & N_{334} \\
= & \frac{384(1-2 c)^{2} \beta v_{*}}{3 \sqrt{1-2 c}(1+2 \sqrt{1-2 c})(1+4 \sqrt{1-2 c})(1+6 \sqrt{1-2 c})(1+8 \sqrt{1-2 c})(1+10 \sqrt{1-2 c})} \\
\times & \left(\left(297(1+\sqrt{1-2 c})-6(191+152 \sqrt{1-2 c}) c+16(69+40 \sqrt{1-2 c}) c^{2}\right)\right. \\
& \times F\left(6,1+\frac{1}{2 \sqrt{1-2 c}} ; 4+\frac{1}{2 \sqrt{1-2 c}} ;-1\right) \\
& -(8 c-3)(-6(4+7 \sqrt{1-2 c})+80 c(1+\sqrt{1-2 c})) \\
& \cdot F\left(6,2+\frac{1}{2 \sqrt{1-2 c}} ; 5+\frac{1}{2 \sqrt{1-2 c}} ;-1\right) \\
& \left.+(3(\sqrt{1-2 c}-1)-2 c(4 \sqrt{1-2 c}-3)) F\left(6,3+\frac{1}{2 \sqrt{1-2 c}} ; 6+\frac{1}{2 \sqrt{1-2 c}} ;-1\right)\right) .
\end{aligned}
$$

According to (2.301), integral $N_{345}$ is

$$
\begin{aligned}
N_{345}= & N_{335} \\
= & -\frac{1024 \sqrt{2}(1-2 c)^{3} \beta}{(1+3 \sqrt{1-2 c})(1+55 \sqrt{1-2 c})(1+7 \sqrt{1-2 c})(1+9 \sqrt{1-2 c})(1+11 \sqrt{1-2 c})(1+13 \sqrt{1-2 c})} \\
& \times\left(\left(252(1+\sqrt{1-2 c})-3(327+263 \sqrt{1-2 c}) c+(954+572 \sqrt{1-2 c}) c^{2}\right)\right. \\
& \times F\left(8, \frac{1}{2}\left(3+\frac{1}{\sqrt{1-2 c}}\right) ; \frac{1}{2}\left(11+\frac{1}{\sqrt{1-2 c}}\right) ;-1\right) \\
& -2(8 c-3)(-8-20 \sqrt{1-2 c}+16 c+39 c \sqrt{1-2 c}) \\
& +F\left(8, \frac{1}{2}\left(5+\frac{1}{\sqrt{1-2 c}}\right) ; \frac{1}{2}\left(13+\frac{1}{\sqrt{1-2 c}}\right) ;-1\right) \\
& +\left(12(\sqrt{1-2 c}-1)-53 c(\sqrt{1-2 c}-1)+c^{2}(60 \sqrt{1-2 c}-58)\right) \\
& \left.\times F\left(8, \frac{1}{2}\left(7+\frac{1}{\sqrt{1-2 c}}\right) ; \frac{1}{2}\left(15+\frac{1}{\sqrt{1-2 c}}\right) ;-1\right)\right) .
\end{aligned}
$$

Substitution of the above formulas into (2.303) gives

$$
N_{34}=-\frac{2}{3} \sqrt{2(1-2 c)}\left(3 \beta v_{*}^{2}-\alpha\right)+\frac{2(4 c-3)}{6 c-3}\left(v_{*}-\beta v_{*}^{3}-T_{0}\right)-N_{344}+N_{345}
$$


whereas substitution of the above formulas into (2.279) gives

$$
\begin{aligned}
N_{3}\left(t_{0}\right)= & -\tilde{A} \sin \left(\omega t_{0}+\kappa\right)+\frac{2}{3} \sqrt{2(1-2 c)}\left(\delta_{11}-\delta_{21}\right)-N_{323}-N_{324} \\
& +\frac{\sqrt{2}}{3(1-2 c)}\left(\alpha_{11}-\alpha_{21}\right)\left(3 \sqrt{1-2 c}+c \psi\left(\frac{1}{4}\left(1+\frac{1}{\sqrt{1-2 c}}\right)\right)-c \psi\left(\frac{1}{4}\left(3+\frac{1}{\sqrt{1-2 c}}\right)\right)\right) \\
& +\frac{4(4 c-3)}{6 c-3}\left(v_{*}-\beta v_{*}^{3}-T_{0}\right)-N_{334}-N_{335}-N_{344}+N_{345} \\
& - \begin{cases}4 T_{0}\left(\bar{N}_{331}\left(t_{1}\right)-\bar{N}_{331}\left(t_{2}\right)+\bar{N}_{341}\left(t_{3}\right)-\bar{N}_{341}\left(t_{4}\right)\right) & \text { for } v_{*}<\frac{1-2 c}{\sqrt{2}} \\
0 & \text { for } v_{*} \geq \frac{1-2 c}{\sqrt{2}} .\end{cases}
\end{aligned}
$$

Finally, the following chaos criterion is obtained:

$$
\begin{aligned}
\tilde{A}>\mid & \frac{2}{3} \sqrt{2(1-2 c)}\left(\delta_{11}-\delta_{21}\right)-N_{323}-N_{324} \\
& +\frac{\sqrt{2}}{3(1-2 c)}\left(\alpha_{11}-\alpha_{21}\right)\left(3 \sqrt{1-2 c}+c \psi\left(\frac{1}{4}\left(1+\frac{1}{\sqrt{1-2 c}}\right)\right)-c \psi\left(\frac{1}{4}\left(3+\frac{1}{\sqrt{1-2 c}}\right)\right)\right) \\
& +\frac{4(4 c-3)}{6 c-3}\left(v_{*}-\beta v_{*}^{3}-T_{0}\right)-N_{334}-N_{335}-N_{344}+N_{345} \\
& - \begin{cases}4 T_{0}\left(\bar{N}_{331}\left(t_{1}\right)-\bar{N}_{331}\left(t_{2}\right)\right) & \text { for } v_{*}<\frac{1-2 c}{\sqrt{2}} \\
0 & \text { for } v_{*} \geq \frac{1-2 c}{\sqrt{2}}\end{cases}
\end{aligned}
$$

According to (2.92), the Melnikov function is defined by

$$
N_{1-}\left(t_{0}\right)=N_{11-}\left(t_{0}\right)+N_{12-}+N_{13-}-N_{14-},
$$

where

$$
\begin{gathered}
N_{11-}\left(t_{0}\right)=N_{11}\left(t_{0}\right)=2 \gamma \int_{0}^{\infty} R(-\sqrt{1-2 c} t) e^{-t} \cos \omega\left(t+t_{0}\right) d t \\
N_{12-}=2 \int_{0}^{\infty}\left(\delta_{1}\left(x_{10}, \dot{x}_{10}\right)+\delta_{2}\left(x_{10}, \dot{x}_{10}\right)\right) e^{-t} R(-\sqrt{1-2 c} t) d t \\
N_{13-}=N_{13}=2 \int_{0}^{\infty} e^{-t} R(-\sqrt{1-2 c} t) T\left(\dot{x}_{01}-v_{*}\right) d t \\
N_{14-}=N_{14}=2 \int_{0}^{\infty} e^{-t} R(-\sqrt{1-2 c} t) T\left(-\dot{x}_{01}-v_{*}\right) d t .
\end{gathered}
$$


The integral for $N_{12-}$ is defined as follows:

$$
N_{12-}=N_{121-}-N_{122-}-N_{123-}-N_{124-} \text {, }
$$

where

$$
\begin{aligned}
& N_{121-}=2 \sqrt{2(1-2 c)}\left(\alpha_{11}+\alpha_{21}\right) \int_{0}^{\infty} \operatorname{sech}(\sqrt{1-2 c} t) e^{-t} R(-\sqrt{1-2 c} t) d t, \\
& N_{122-}=2 \sqrt{2}(1-2 c)\left(\delta_{11}+\delta_{21}\right) \int_{0}^{\infty} \operatorname{sech}(\sqrt{1-2 c} t) \operatorname{tgh}(\sqrt{1-2 c} t) e^{-t} R(-\sqrt{1-2 c} t) d t, \\
& N_{123-}=4(1-2 c)^{2}\left(\delta_{12}+\delta_{22}\right) \int_{0}^{\infty} \operatorname{sech}^{2}(\sqrt{1-2 c} t) \operatorname{tgh}^{2}(\sqrt{1-2 c} t) e^{-t} R(-\sqrt{1-2 c} t) \\
& N_{124-}=4 \sqrt{2}(1-2 c)^{3}\left(\delta_{13}+\delta_{23}\right) \int_{0}^{\infty} \operatorname{sech}^{3}(\sqrt{1-2 c} t) \operatorname{tgh}^{3}(\sqrt{1-2 c} t) e^{-t} R(-\sqrt{1-2 c} t) d t .
\end{aligned}
$$

The computations give

$$
\begin{aligned}
& N_{121-}=\frac{\sqrt{2}}{3(2 c-1)}\left(\alpha_{21}+\alpha_{11}\right)\left(3 \sqrt{1-2 c}+c \psi\left(\frac{1}{4}\left(1+\frac{1}{\sqrt{1-2 c}}\right)\right)-c \psi\left(\frac{1}{4}\left(3+\frac{1}{\sqrt{1-2 c}}\right)\right)\right) \\
& N_{122-}=-\frac{2 \sqrt{2(1-2 c)}}{3}\left(\delta_{11}+\delta_{21}\right) \\
& N_{123-}=-\frac{128(1-2 c)^{3 / 2}\left(\delta_{22}+\delta_{12}\right)}{3(1+2 \sqrt{1-2 c})(1+4 \sqrt{1-2 c})(1+6 \sqrt{1-2 c})(1+8 \sqrt{1-2 c})(1+10 \sqrt{1-2 c})} \\
& \times\left(\left(-6 a(191+152 \sqrt{1-2 c})+297(1+\sqrt{1-2 c})+16(69+40 \sqrt{1-2 c}) c^{2}\right)\right. \\
& \times F\left(6, \frac{1}{2}\left(2+\frac{1}{\sqrt{1-2 c}}\right) ; \frac{1}{2}\left(8+\frac{1}{\sqrt{1-2 c}}\right) ;-1\right) \\
& +(3+8 c)((-6(4+7 \sqrt{1-2 c})+16 c(3+5 \sqrt{1-2 c})) \\
& \times F\left(6, \frac{1}{2}\left(4+\frac{1}{\sqrt{1-2 c}}\right) ; \frac{1}{2}\left(10+\frac{1}{\sqrt{1-2 c}}\right) ;-1\right) \\
& +(-3+3 \sqrt{1-2 c}+6 c-8 c \sqrt{1-2 c}) \\
& \left.\left.F\left(6, \frac{1}{2}\left(6+\frac{1}{\sqrt{1-2 c}}\right) ; \frac{1}{2}\left(12+\frac{1}{\sqrt{1-2 c}}\right) ;-1\right)\right)\right),
\end{aligned}
$$




$$
\begin{aligned}
N_{124-}= & \frac{1024 \sqrt{2}(1-2 c)^{3}\left(\delta_{23}+\delta_{13}\right)}{(1+3 \sqrt{1-2 c})(1+5 \sqrt{1-2 c})(1+7 \sqrt{1-2 c})(1+9 \sqrt{1-2 c})(1+11 \sqrt{1-2 c})(1+13 \sqrt{1-2 c})} \\
\times( & \left(252(1+\sqrt{1-2 c})-3 c(327+263 \sqrt{1-2 c})+(954+572 \sqrt{1-2 c}) c^{2}\right) \\
& \times F\left(8, \frac{1}{2}\left(3+\frac{1}{\sqrt{1-2 c}}\right) ; \frac{1}{2}\left(11+\frac{1}{\sqrt{1-2 c}}\right) ;-1\right) \\
& +2(3+8 c)(-8-20 \sqrt{1-2 c}+16 c+39 c \sqrt{1-2 c}) \\
& F\left(8, \frac{1}{2}\left(5+\frac{1}{\sqrt{1-2 c}}\right) ; \frac{1}{2}\left(13+\frac{1}{\sqrt{1-2 c}}\right) ;-1\right) \\
& +\left(12(-1+\sqrt{1-2 c})-53 c(-1+\sqrt{1-2 c})+(-58+60 \sqrt{1-2 c}) c^{2}\right) \\
& \left.\times F\left(8, \frac{1}{2}\left(7+\frac{1}{\sqrt{1-2 c}}\right) ; \frac{1}{2}\left(15+\frac{1}{\sqrt{1-2 c}}\right) ;-1\right)\right)
\end{aligned}
$$

and hence

$$
\begin{aligned}
N_{12-}= & \frac{\sqrt{2}}{3(2 c-1)}\left(\alpha_{21}+\alpha_{11}\right)\left(3 \sqrt{1-2 c}+c \psi\left(\frac{1}{4}\left(1+\frac{1}{\sqrt{1-2 c}}\right)\right)-c \psi\left(\frac{1}{4}\left(3+\frac{1}{\sqrt{1-2 c}}\right)\right)\right) \\
& +\frac{2}{3} \sqrt{2(1-2 c)}\left(\delta_{11}+\delta_{21}\right)-N_{123}-N_{124} .
\end{aligned}
$$

Substitution of (2.215), (2.230), (2.242), and (2.318) into (2.313) yields

$$
\begin{aligned}
N_{1-}\left(t_{0}\right)= & \tilde{A} \sin \left(\omega t_{0}-\kappa\right)+\frac{2}{3} \sqrt{2(1-2 c)}\left(\delta_{11}+\delta_{21}\right)-N_{123}-N_{124} \\
& +\frac{\sqrt{2}}{3(2 c-1)}\left(\alpha_{21}+\alpha_{11}\right)\left(3 \sqrt{1-2 c}+c \psi\left(\frac{1}{4}\left(1+\frac{1}{\sqrt{1-2 c}}\right)\right)-c \psi\left(\frac{1}{4}\left(3+\frac{1}{\sqrt{1-2 c}}\right)\right)\right) \\
& -\frac{4}{3} \sqrt{2(1-2 c)}\left(3 \beta v_{*}^{2}-\alpha\right)-N_{134}-N_{135}+N_{144}-N_{145} \\
& - \begin{cases}4 T_{0}\left(-\bar{N}_{141}\left(t_{3}\right)+\bar{N}_{141}\left(t_{4}\right)\right) & \text { for } v_{*}<\sqrt{\frac{1-2 c}{2}} \\
0 & \text { for } v_{*} \geq \sqrt{\frac{1-2 c}{2}} .\end{cases}
\end{aligned}
$$


The criterion of chaos is defined by the following inequality:

$$
\begin{aligned}
\tilde{A}>\mid & \frac{2}{3} \sqrt{2(1-2 c)}\left(\delta_{11}+\delta_{21}\right)-N_{123}-N_{124} \\
& +\frac{\sqrt{2}}{3(2 c-1)}\left(\alpha_{21}+\alpha_{11}\right)\left(3 \sqrt{1-2 c}+c \psi\left(\frac{1}{4}\left(1+\frac{1}{\sqrt{1-2 c}}\right)\right)-c \psi\left(\frac{1}{4}\left(3+\frac{1}{\sqrt{1-2 c}}\right)\right)\right) \\
& -\frac{4}{3} \sqrt{2(1-2 c)}\left(3 \beta v_{*}^{2}-\alpha\right)-N_{134}-N_{135}+N_{144}-N_{145} \\
& - \begin{cases}4 T_{0}\left(-\bar{N}_{141}\left(t_{3}\right)+\bar{N}_{141}\left(t_{4}\right)\right) & \text { for } v_{*}<\sqrt{\frac{1-2 c}{2}} \\
0 & \text { for } v_{*} \geq \sqrt{\frac{1-2 c}{2}}\end{cases}
\end{aligned}
$$

According to (2.95), the Melnikov function is

$$
N_{2-}\left(t_{0}\right)=N_{21-}\left(t_{0}\right)+N_{22-}-N_{23-}-N_{24-},
$$

where

$$
\begin{gathered}
N_{21-}\left(t_{0}\right)=N_{21}\left(t_{0}\right)=2 A \gamma \int_{-\infty}^{\infty} \dot{r}(\sqrt{1-2 c} t) \cos \omega\left(t+t_{0}\right) d t \\
N_{22-}=N_{22}=2 A \int_{-\infty}^{\infty}\left(\delta_{1}\left(x_{10}, \dot{x}_{10}\right)-\delta_{2}\left(x_{10}, \dot{x}_{10}\right)\right) \dot{r}(\sqrt{1-2 c} t) d t \\
N_{23-}=N_{23}=2 A \int_{-\infty}^{\infty} \dot{r}(\sqrt{1-2 c} t) T\left(\dot{x}_{01}-v_{*}\right) d t \\
N_{24-}=N_{24}=2 A \int_{-\infty}^{\infty} \dot{r}(\sqrt{1-2 c} t) T\left(-\dot{x}_{01}-v_{*}\right) d t
\end{gathered}
$$

Substitution of (2.250), (2.254), (2.266), and (2.276) into (2.321) yields the next Melnikov function defined in the following way:

$$
\begin{aligned}
N_{2-}\left(t_{0}\right)= & \frac{2 \pi A \gamma \omega}{\sqrt{1-2 c}} \operatorname{sech}\left(\frac{\pi \omega}{2 \sqrt{1-2 c}}\right) \sin \omega t_{0} \\
& +\frac{4 A}{3}\left(\sqrt{2(1-2 c)}\left(\delta_{11}-\delta_{21}\right)+\frac{4}{5}(1-2 c)^{3 / 2}\left(\delta_{12}-\delta_{22}\right)+\frac{4 \sqrt{2}}{5}(1-2 c)^{5 / 2}\left(\delta_{13}-\delta_{23}\right)\right) \\
& + \begin{cases}8 A T_{0}\left(\sqrt{\frac{1}{2}-\sqrt{\frac{1}{4}-\frac{v_{*}^{2}}{2(1-2 c)^{2}}}}-\sqrt{\frac{1}{2}+\sqrt{\frac{1}{4}-\frac{v_{*}^{2}}{2(1-2 c)^{2}}}}\right) & \text { for } v_{*}<\frac{1-2 c}{\sqrt{2}} \\
0 & \text { for } v_{*} \geq \frac{1-2 c}{\sqrt{2}} .\end{cases}
\end{aligned}
$$


The condition of intersection of stable and unstable manifolds is as follows:

$$
\begin{aligned}
& \frac{2 \pi|A| \gamma \omega}{\sqrt{1-2 c}} \operatorname{sech}\left(\frac{\pi \omega}{2 \sqrt{1-2 c}}\right) \\
& > \\
& \quad \frac{4 A}{3}\left(\sqrt{2(1-2 c)}\left(\delta_{11}-\delta_{21}\right)+\frac{4}{5}(1-2 c)^{3 / 2}\left(\delta_{12}-\delta_{22}\right)+\frac{4 \sqrt{2}}{5}(1-2 c)^{5 / 2}\left(\delta_{13}-\delta_{23}\right)\right) \\
& + \begin{cases}8 A T_{0}\left(\sqrt{\frac{1}{2}-\sqrt{\frac{1}{4}-\frac{v_{*}^{2}}{2(1-2 c)^{2}}}}-\sqrt{\frac{1}{2}+\sqrt{\frac{1}{4}-\frac{v_{*}^{2}}{2(1-2 c)^{2}}}}\right) & \text { for } v_{*}<\frac{1-2 c}{\sqrt{2}} \\
0 \quad \text { for } v_{*} \geq \frac{1-2 c}{\sqrt{2}}\end{cases}
\end{aligned}
$$

According to (2.98), the Melnikov function has the following form:

$$
N_{3-}\left(t_{0}\right)=-N_{31-}\left(t_{0}\right)-N_{32-}+N_{33-}+N_{34-},
$$

where

$$
\begin{gathered}
N_{31-}\left(t_{0}\right)=N_{31}\left(t_{0}\right)=2 \gamma \int_{-\infty}^{0} R(\sqrt{1-2 c} t) e^{t} \cos \omega\left(t+t_{0}\right) d t \\
N_{32-}=N_{32}=2 \int_{-\infty}^{0}\left(\delta_{1}\left(x_{10}, \dot{x}_{10}\right)-\delta_{2}\left(x_{10}, \dot{x}_{10}\right)\right) e^{t} R(\sqrt{1-2 c} t) d t \\
N_{33-}=N_{33}=2 \int_{-\infty}^{0} e^{t} R(\sqrt{1-2 c} t) T\left(\dot{x}_{01}-v_{*}\right) d t \\
N_{34-}=N_{34}=2 \int_{-\infty}^{0} e^{t} R(\sqrt{1-2 c} t) T\left(-\dot{x}_{01}-v_{*}\right) d t .
\end{gathered}
$$

Substitution of the above formulas into (2.325) gives

$$
\begin{aligned}
N_{3-}\left(t_{0}\right)= & -\tilde{A} \sin \left(\omega t_{0}+\kappa\right)-\frac{2}{3} \sqrt{2(1-2 c)}\left(\delta_{11}-\delta_{21}\right)-N_{323}-N_{324} \\
& -\frac{\sqrt{2}}{3(1-2 c)}\left(\alpha_{11}-\alpha_{21}\right)\left(3 \sqrt{1-2 c}+c \psi\left(\frac{1}{4}\left(1+\frac{1}{\sqrt{1-2 c}}\right)\right)-c \psi\left(\frac{1}{4}\left(3+\frac{1}{\sqrt{1-2 c}}\right)\right)\right) \\
& +\frac{4(4 c-3)}{6 c-3}\left(v_{*}-\beta v_{*}^{3}-T_{0}\right)-N_{334}-N_{335}-N_{344}+N_{345} \\
& - \begin{cases}4 T_{0}\left(\bar{N}_{331}\left(t_{1}\right)-\bar{N}_{331}\left(t_{2}\right)+\bar{N}_{341}\left(t_{3}\right)-\bar{N}_{341}\left(t_{4}\right)\right) & \text { for } v_{*}<\frac{1-2 c}{\sqrt{2}}, \\
0 & \text { for } v_{*} \geq \frac{1-2 c}{\sqrt{2}} .\end{cases}
\end{aligned}
$$




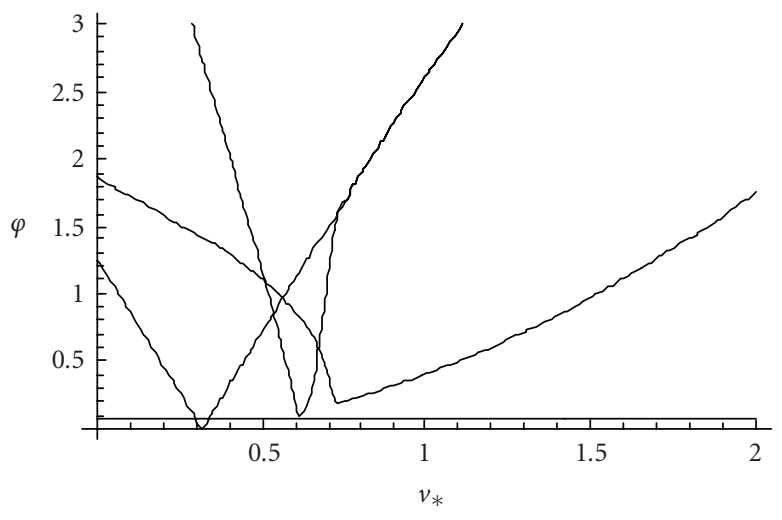

Figure 3.1. Thresholds of chaos $\gamma=\gamma\left(v_{*}\right)$ for $\delta_{1}=0,1 ; \delta_{2}=0,2 ; c=0,3 ; T_{0}=0,3 ; \alpha=0,2 ; \beta=0,1$; $v_{*}=0,2 ; \omega=1$.

Finally, the chaos criterion is given by the following inequality:

$$
\begin{aligned}
\tilde{A}>\mid & -\frac{2}{3} \sqrt{2(1-2 c)}\left(\delta_{11}-\delta_{21}\right)-N_{323}-N_{324} \\
& -\frac{\sqrt{2}}{3(1-2 c)}\left(\alpha_{11}-\alpha_{21}\right)\left(3 \sqrt{1-2 c}+c \psi\left(\frac{1}{4}\left(1+\frac{1}{\sqrt{1-2 c}}\right)\right)-c \psi\left(\frac{1}{4}\left(3+\frac{1}{\sqrt{1-2 c}}\right)\right)\right) \\
& +\frac{4(4 c-3)}{6 c-3}\left(v_{*}-\beta v_{*}^{3}-T_{0}\right)-N_{334}-N_{335}-N_{344}+N_{345} \\
& - \begin{cases}4 T_{0}\left(\bar{N}_{331}\left(t_{1}\right)-\bar{N}_{331}\left(t_{2}\right)+\bar{N}_{341}\left(t_{3}\right)-\bar{N}_{341}\left(t_{4}\right)\right) & \text { for } v_{*}<\frac{1-2 c}{\sqrt{2}} \\
0 & \text { for } v_{*} \geq \frac{1-2 c}{\sqrt{2}}\end{cases}
\end{aligned}
$$

\section{Numerical simulations}

In Figure 3.1, chaotic thresholds $\gamma=\gamma\left(v_{*}\right)$ generated by chaos criteria governed by formulas (2.115), (2.144), (2.174), (2.178), (2.198), and (2.207) and associated with collapsing of the homoclinic orbits $q_{01,2}(t)$ governed by $(2.18)$ are reported.

For the parameters $\delta_{1}=0,1 ; \delta_{2}=0,2 ; c=0,3 ; T_{0}=0,3 ; \alpha=0,2 ; \beta=0,1 ; v_{*}=0,2$; $\omega=1$, critical values of the parameter $\gamma$ are as follows: $\gamma_{\mathrm{cr} 1} \approx 0,071, \gamma_{\mathrm{cr} 2} \approx 0,47, \gamma_{\mathrm{cr}} \approx$ $1,59, \gamma_{\mathrm{cr} 4} \approx 3,76$. We assume that the coupling stiffness has the form either $k_{0}(z)=z^{5}$ or $k_{0}(z)=z^{3}$. In Figure 3.2, bifurcation diagrams of the investigated system in the case 


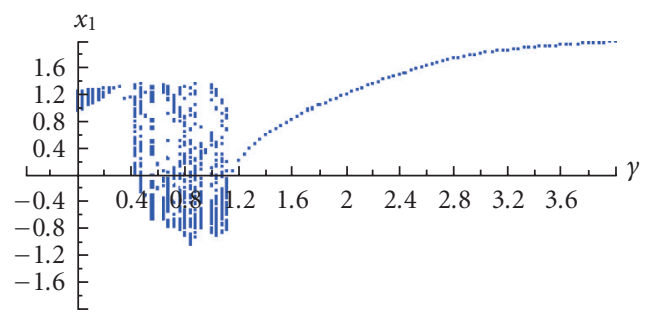

(a)

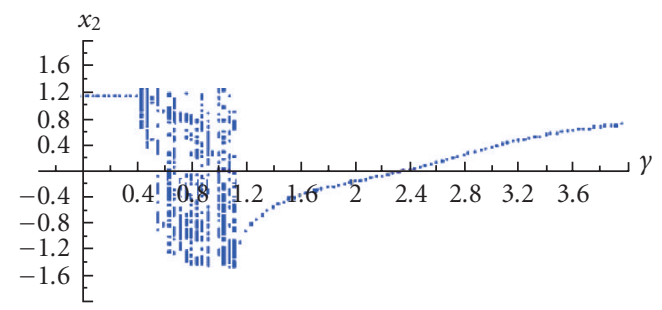

(b)

Figure 3.2. Bifurcation diagrams for $k_{0}(z)=z^{5}$ and for $\delta_{1}=0,1 ; \delta_{2}=0,2 ; c=0,3 ; T_{0}=0,3 ; \alpha=0,2$; $\beta=0,1 ; v_{*}=0,2 ; \omega=1$.

$k_{0}(z)=z^{5}$ and for $\delta_{1}=0,1 ; \delta_{2}=0,2 ; c=0,3 ; T_{0}=0,3 ; \alpha=0,2 ; \beta=0,1 ; v_{*}=0,2 ; \omega=1$ are shown. Amplitude of excitation $\gamma$ serves as the bifurcation parameter.

One may observe bifurcation in vicinity of the critical value $\gamma_{\mathrm{cr} 2}$, which takes place in the interval $(0,40,0,44)$. Figure 3.3 displays phase plots and Poincaré maps for $k_{0}(z)=$ $z^{5}$ and for $\gamma=0,40 ; \delta_{1}=0,1 ; \delta_{2}=0,2 ; c=0,3 ; T_{0}=0,3 ; \alpha=0,2 ; \beta=0,1 ; \nu_{*}=0,2$; $\omega=1$. Regular dynamics is observed. Increase of the excitation amplitude up to $\gamma=0,44$ remaining fixed other parameters results in occurrence of chaotic dynamics.

In Figure 3.4, phase plots and Poincaré maps for $k_{0}(z)=z^{5}$ and for $\gamma=0,44 ; \delta_{1}=0,1$; $\delta_{2}=0,2 ; c=0,3 ; T_{0}=0,3 ; \alpha=0,2 ; \beta=0,1 ; v_{*}=0,2 ; \omega=1$ are shown.

The system moves in a chaotic manner and chaotic attractors with complex and stickslip structures are exhibited.

Let us analyze now dynamics of the investigated system for the coupling term $k_{0}(z)=$ $z^{3}$. Note that owing to the Melnikov-Gruendler approach applied to the Duffing oscillator, the same transition into chaos is now also predicted as it was for the case of $k_{0}(z)=z^{5}$.

In Figure 3.5, bifurcational diagrams for the parameter $\gamma$ for the stiffness $k_{0}(z)=z^{3}$ and the fixed parameters $\delta_{1}=0,1 ; \delta_{2}=0,2 ; c=0,3 ; T_{0}=0,3 ; \alpha=0,2 ; \beta=0,1 ; v_{*}=0,2$; $\omega=1$ are shown.

It is confirmed that the homoclinic bifurcation takes place in the vicinity of $\gamma_{\mathrm{cr} 2}$. In Figure 3.6, phase plots and Poincaré maps for $k_{0}(z)=z^{3}$ and for $\gamma=0,44 ; \delta_{1}=0,1 ; \delta_{2}=$ 0,$2 ; c=0,3 ; T_{0}=0,3 ; \alpha=0,2 ; \beta=0,1 ; v_{*}=0,2 ; \omega=1$ are reported. 
72 Stick-slip chaos in a self-excited oscillator

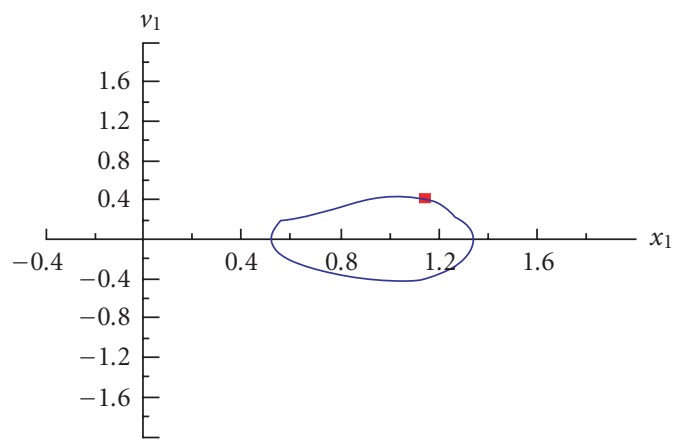

(a)

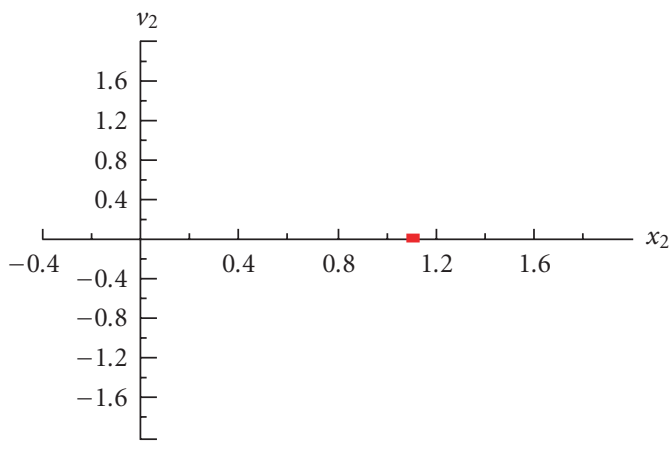

(b)

Figure 3.3. Phase plots and Poincaré maps for $k_{0}(z)=z^{5}$ and for $\gamma=0,40 ; \delta_{1}=0,1 ; \delta_{2}=0,2 ; c=0,3$; $T_{0}=0,3 ; \alpha=0,2 ; \beta=0,1 ; v_{*}=0,2 ; \omega=1$.

For $\gamma=0,44$, the system moves on the periodic orbit with excitation frequency. In Figure 3.7, phase plots and Poincaré maps for $k_{0}(z)=z^{3}$ and for $\gamma=0,48 ; \delta_{1}=0,1 ; \delta_{2}=$ 0,$2 ; c=0,3 ; T_{0}=0,3 ; \alpha=0,2 ; \beta=0,1 ; v_{*}=0,2 ; \omega=1$ are shown.

Increase of $\gamma$ up to $\gamma=0,48$ yields occurrence of a chaotic attractor.

In Figure 3.8, chaotic orbits yielded by destruction of the homoclinic orbits $q_{01,2}(t)$ and $q_{03,4}(t)$ and obtained by (2.18) and (2.19), respectively, for the coupling $k_{0}(z)=z$ are displayed.

In this case, additional critical values of the $\gamma$ parameter are found: $\gamma_{\mathrm{cr} 6} \approx 0,072, \gamma_{\mathrm{cr} 7} \approx$ $1,19, \gamma_{\mathrm{cr} 8} \approx 1,32, \gamma_{\mathrm{cr} 9} \approx 1,48, \gamma_{\mathrm{cr} 10} \approx 1,54$. They correspond to collapse of the homoclinic orbits $q_{03,4}(t)$. In Figure 3.9, the associated bifurcational diagrams for $k_{0}(z)=z$ and $\delta_{1}=$ 0,$1 ; \delta_{2}=0,2 ; c=0,3 ; T_{0}=0,3 ; \alpha=0,2 ; \beta=0,1 ; v_{*}=0,2 ; \omega=1$ are shown.

Since in the considered case we have ten different critical values of the parameter $\gamma$, one may expect much more complex chaotic dynamics. In Figure 3.10, phase projections and 


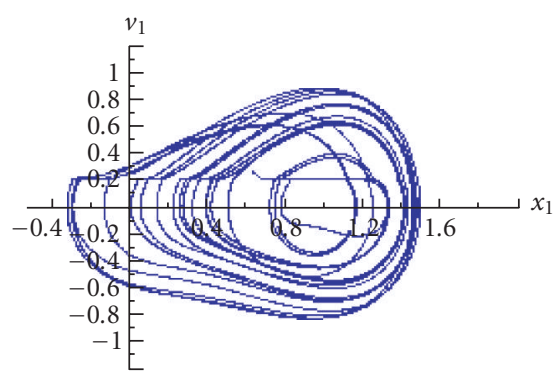

(a)

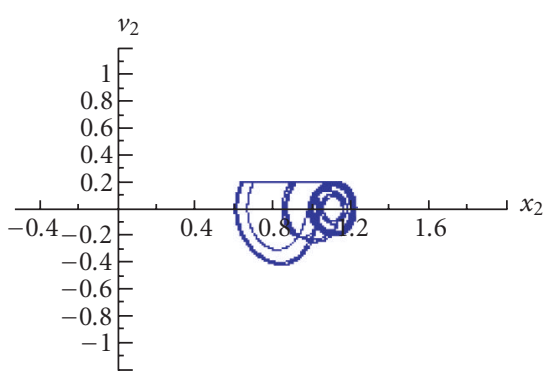

(c)

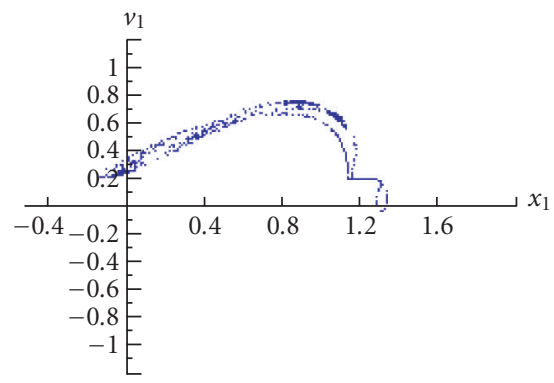

(b)

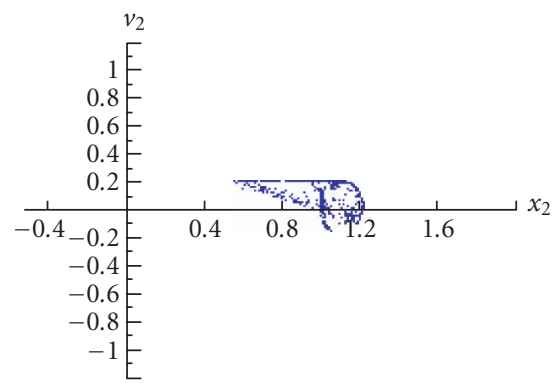

(d)

Figure 3.4. Phase plots and Poincaré maps for $k_{0}(z)=z^{5}$ and for $\gamma=0,44 ; \delta_{1}=0,1 ; \delta_{2}=0,2 ; c=0,3$; $T_{0}=0,3 ; \alpha=0,2 ; \beta=0,1 ; v_{*}=0,2 ; \omega=1$.

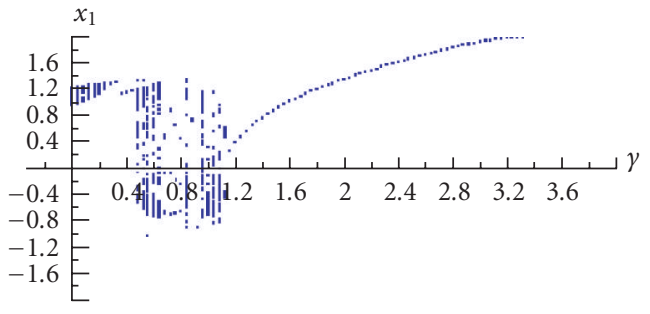

(a)

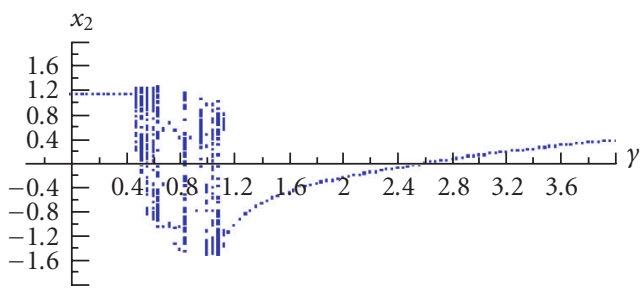

(b)

Figure 3.5. Bifurcation diagrams for $k_{0}(z)=z^{3}$ and for $\delta_{1}=0,1 ; \delta_{2}=0,2 ; c=0,3 ; T_{0}=0,3 ; \alpha=0,2$; $\beta=0,1 ; v_{*}=0,2 ; \omega=1$. 
74 Stick-slip chaos in a self-excited oscillator

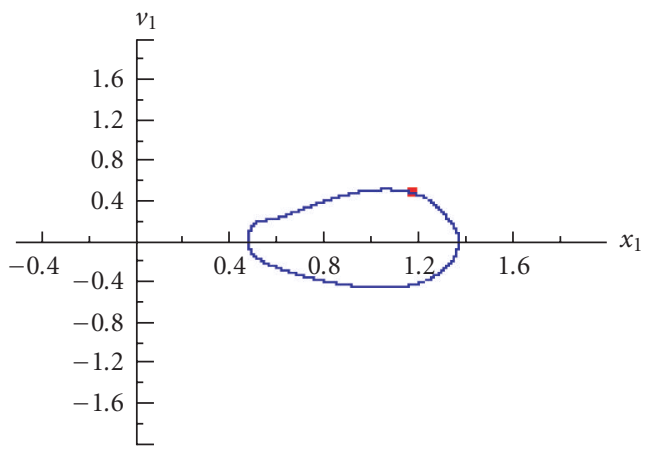

(a)

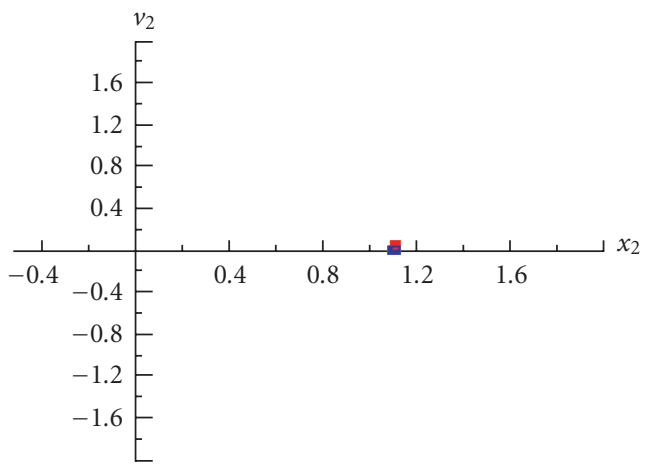

(b)

Figure 3.6. Phase plots and Poincaré maps for $k_{0}(z)=z^{3}$ and for $\gamma=0,44 ; \delta_{1}=0,1 ; \delta_{2}=0,2 ; c=0,3$; $T_{0}=0,3 ; \alpha=0,2 ; \beta=0,1 ; v_{*}=0,2 ; \omega=1$.

Poincaré maps of the studied for $k_{0}(z)=z$ and for $\gamma=0,60 ; \delta_{1}=0,1 ; \delta_{2}=0,2 ; c=0,3$; $T_{0}=0,3 ; \alpha=0,2 ; \beta=0,1 ; v_{*}=0,2 ; \omega=1$ are shown.

In the beginning, the system moves in a periodic manner in the vicinity of one of its equilibrium position. In Figure 3.11, phase plots and Poincaré maps for $k_{0}(z)=z$ and for $\gamma=0,61 ; \delta_{1}=0,1 ; \delta_{2}=0,2 ; c=0,3 ; T_{0}=0,3 ; \alpha=0,2 ; \beta=0,1 ; v_{*}=0,2 ; \omega=1$ are reported.

Increase of the control parameter $\gamma$ up to $\gamma=0,61$ results in a qualitative change of the phase portraits. Amplitudes of oscillations increased, and the externally excited mass moves between previous three equilibrium positions in a complex chaotic manner.

\section{Concluding remarks}

The carried out Melnikov-Gruendler method of analytical and numerical analysis of the coupled self-excited oscillators included arbitrary type of coupling elements. In general, 


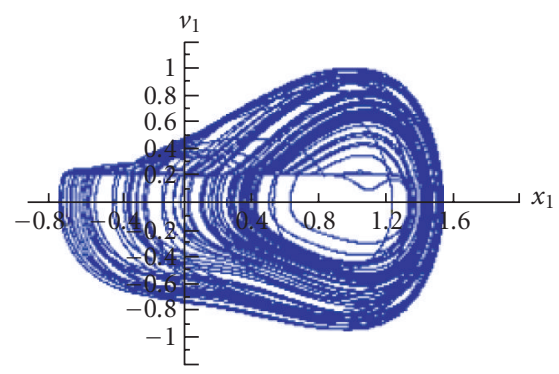

(a)

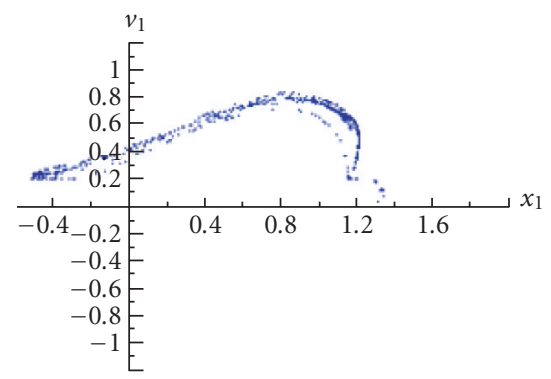

(b)

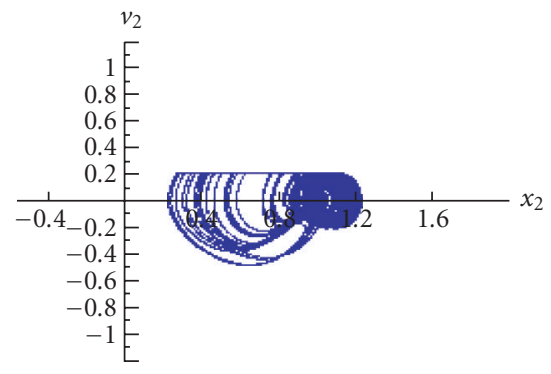

(c)

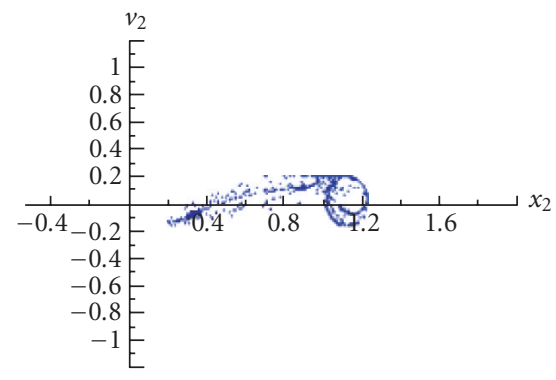

(d)

Figure 3.7. Phase plots and Poincaré maps for $k_{0}(z)=z^{3}$ and for $\gamma=0,48 ; \delta_{1}=0,1 ; \delta_{2}=0,2 ; c=0,3$; $T_{0}=0,3 ; \alpha=0,2 ; \beta=0,1 ; v_{*}=0,2 ; \omega=1$. 
76 Stick-slip chaos in a self-excited oscillator

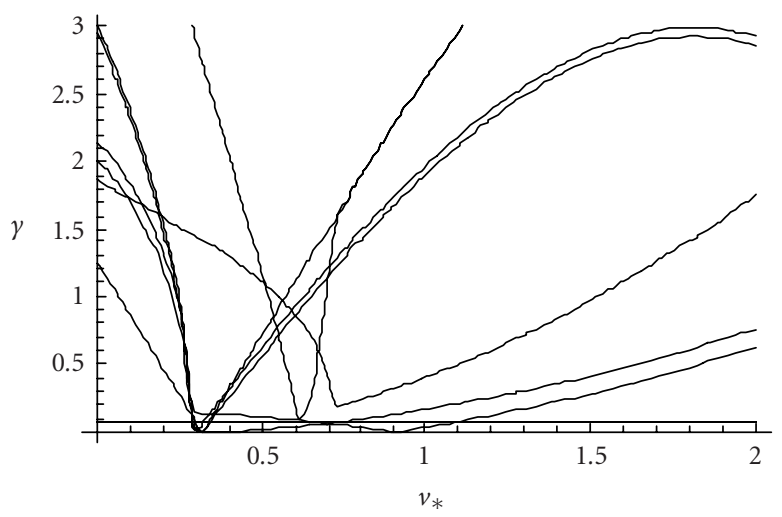

Figure 3.8. Chaotic thresholds for $\gamma=\gamma\left(v_{*}\right), k_{0}(z)=z$ and for $\delta_{1}=0,1 ; \delta_{2}=0,2 ; c=0,3 ; T_{0}=0,3$; $\alpha=0,2 ; \beta=0,1 ; \omega=1$.

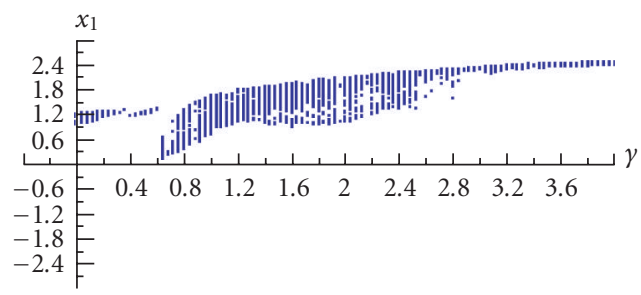

(a)

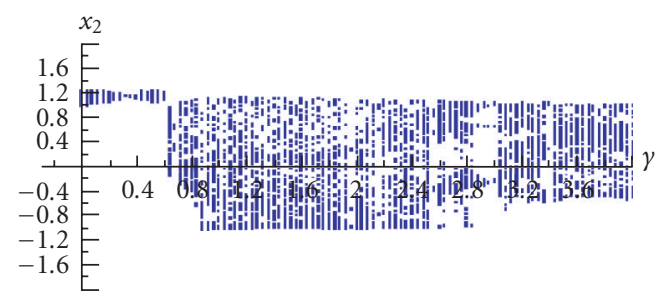

(b)

Figure 3.9. Bifurcational diagrams for $k_{0}(z)=z$ and for $\delta_{1}=0,1 ; \delta_{2}=0,2 ; c=0,3 ; T_{0}=0,3 ; \alpha=0,2$; $\beta=0,1 ; v_{*}=0,2 ; \omega=1$.

two homoclinic orbits exist which yield six chaos critera. Furthermore, depending on the chosen type of coupling elements, one may also expect additional homoclinic orbits associated with additionally found two more homoclinic orbits which yielded six more chaotic thresholds. In result practically homogeneous wide chaotic zone occurred in the bifurcation diagram with the existence of narrow periodic windows. 


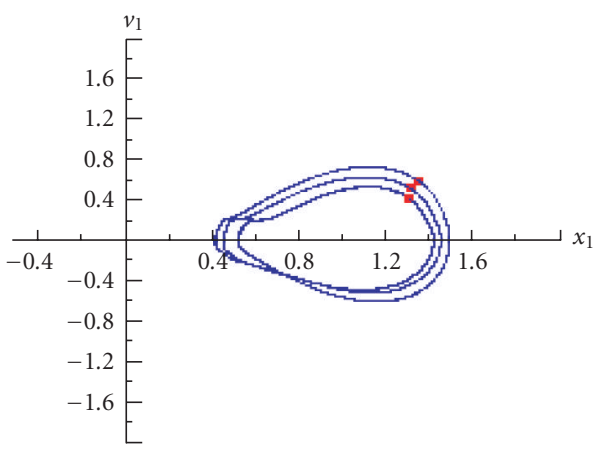

(a)

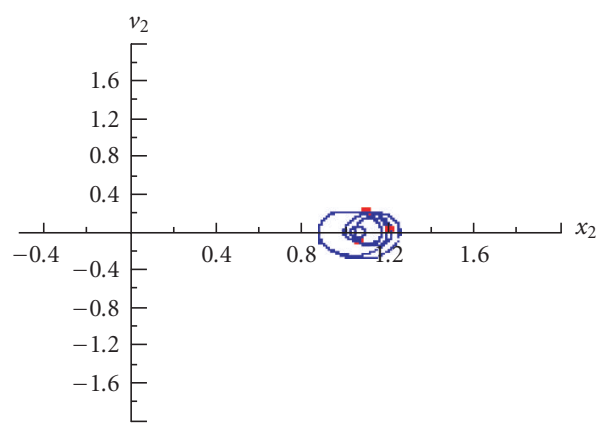

(b)

Figure 3.10. Phase plots and Poincaré maps for $k_{0}(z)=z$ and for $\gamma=0,60 ; \delta_{1}=0,1 ; \delta_{2}=0,2 ; c=0,3$; $T_{0}=0,3 ; \alpha=0,2 ; \beta=0,1 ; v_{*}=0,2 ; \omega=1$.

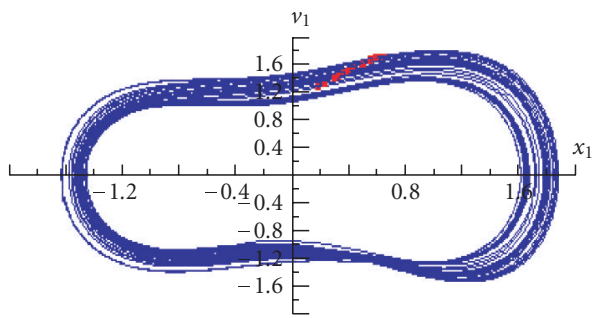

(a)

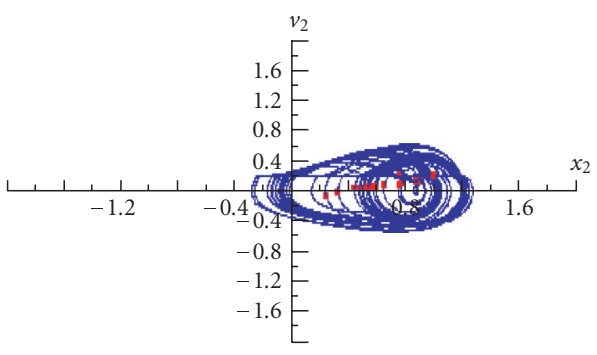

(c)

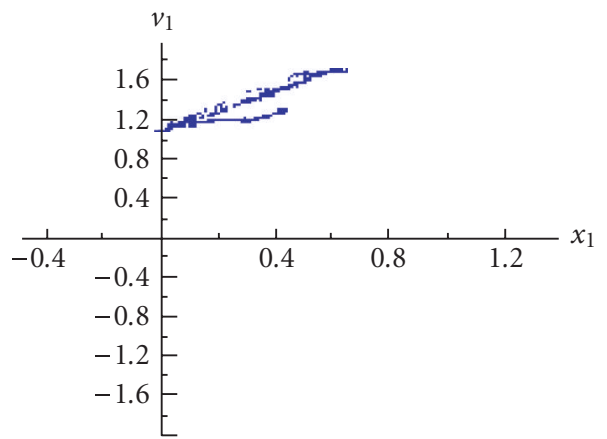

(b)

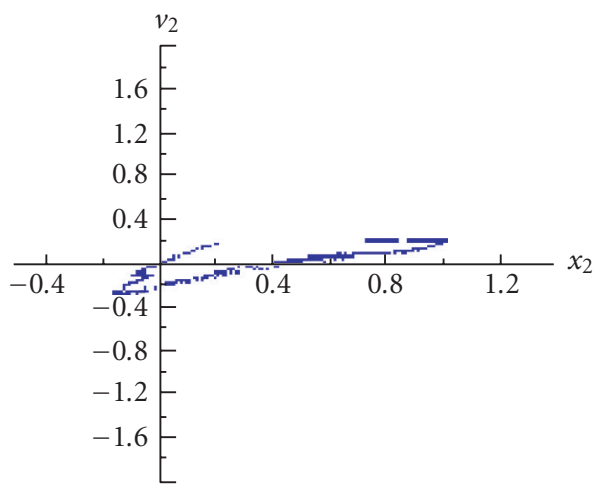

(d)

Figure 3.11. Phase plots and Poincaré maps for $k_{0}(z)=z$ and for $\gamma=0,61 ; \delta_{1}=0,1 ; \delta_{2}=0,2 ; c=0,3$; $T_{0}=0,3 ; \alpha=0,2 ; \beta=0,1 ; v_{*}=0,2 ; \omega=1$. 


\section{Acknowledgment}

This work has been partially supported by the Polish Ministry of Education and Science (Grant no. 4 T07A 031 28).

\section{References}

[1] J. Awrejcewicz, Analysis of self-excited vibration due to dry friction in a system with two degrees of freedom, Scientific Bulletin of Technical University of Lodz, Mechanics 82 (1993), 921-931.

[2] __ Oscillations of Discrete Deterministic Systems, WNT, Warsaw, 1996.

[3] J. Awrejcewicz and L. Dzyubak, Stick-slip chaotic oscilations in a quasi-autonomus mechanical system, International Journal of Nonlinear Sciences and Numerical Simulations 4 (2003), 155160.

[4] J. Awrejcewicz and M. Holicke, Detection of stick-slip chaotic oscillations using Melnikov's method, Proceedings of the International Conference on Nonlinearity, Bifurcation and Chaos: The Doors to the Future (ICNBC '96), Łódź, September 1996, pp. 71-74.

[5] _ Melnikov's method and stick-slip chaotic oscillations in very weakly forced mechanical systems, International Journal of Bifurcation and Chaos 9 (1999), no. 3, 505-518.

[6] J. Awrejcewicz and P. Olejnik, Stick-slip dynamics of a two-degree-of-freedom system, International Journal of Bifurcation and Chaos 13 (2003), no. 4, 843-861.

[7] J. Awrejcewicz and Yu. Pyryev, Influence of tribilogical processes on a chaotic motions of a bush in a cylinder-bush system, Meccanica 38 (2003), no. 6, 749-761.

[8] J. Awrejcewicz and D. Sendkowski, Stick-slip chaos prediction in a two degrees-of-freedom mechanical system with friction, Proceedings of the 7th Conference on Dynamical Systems - Theory and Applications (J. Awrejcewicz, A. Owczarek, and J. Mrozowski, eds.), Łódź, December 2003, pp. $145-152$.

[9] G. Białkowski, Classical Mechanics, PWN, Warsaw, 1975.

[10] V. Brunsden and P. Holmes, Power spectra of strange attractors near homoclinic orbits, Physical Review Letters 58 (1987), no. 17, 1699-1702.

[11] A. R. Bulsara, W. C. Schieve, and E. W. Jacobs, Homoclinic chaos in systems perturbed by weak Langevin noise, Physical Review. A. Third Series 41 (1990), no. 2, 668-681.

[12] U. Galvanetto, S. R. Bishop, and L. Briseghella, Mechanical stick-slip vibrations, International Journal of Bifurcation and Chaos 5 (1995), no. 3, 637-651.

[13] C. Georghiou, Solution to problem 83-10, SIAM Review 26 (1984), no. 2, 282-283.

[14] J. Gruendler, The existence of homoclinic orbits and the method of Melnikov for systems in $R^{n}$, SIAM Journal on Mathematical Analysis 16 (1985), no. 5, 907-931.

[15] _ Homoclinic solutions for autonomous dynamical systems in arbitrary dimension, SIAM Journal on Mathematical Analysis 23 (1992), no. 3, 702-721.

[16] _ Homoclinic solutions for autonomous ordinary differential equations with nonautonomous perturbations, Journal of Differential Equations 122 (1995), no. 1, 1-26.

[17] J. Guckenheimer and P. Holmes, Nonlinear Oscillations, Dynamical Systems, and Bifurcations of Vector Fields, Applied Mathematical Sciences, vol. 42, Springer, New York, 1983.

[18] P. Holmes, A nonlinear oscillator with a strange attractor, Philosophical Transactions of the Royal Society of London 292 (1979), no. 1394, 419-448.

[19] P. Holmes and J. E. Marsden, Melnikov's method and Arnol'd diffusion for perturbations of integrable Hamiltonian systems, Journal of Mathematical Physics 23 (1982), no. 4, 669-675.

[20] B.-P. Koch and R. W. Leven, Subharmonic and homoclinic bifurcations in a parametrically forced pendulum, Physica D. Nonlinear Phenomena 16 (1985), no. 1, 1-13.

[21] A. Mielke and P. Holmes, Spatially complex equilibria of buckled rods, Archive for Rational Mechanics and Analysis 101 (1988), no. 4, 319-348. 
[22] F. C. Moon, Experiments on chaotic motions of a forced nonlinear oscilator: strange attractors, Journal of Applied Mechanics 8 (1980), 638-644.

[23] F. C. Moon and P. Holmes, A magnetoelastic strange attractor, Journal of Sound and Vibration 65 (1979), no. 2, 275-296.

[24] F. C. Moon and G.-X. Li, Fractal basin boundaries and homoclinic orbits for periodic motion in a two-well potential, Physical Review Letters 55 (1985), no. 14, 1439-1442.

[25] K. Popp and P. Stetler, Nonlinear Oscillations of Structures Induced by Dry Friction, Springer, Berlin, 1989.

[26] F. M. A. Salam, The Mel'nikov technique for highly dissipative systems, SIAM Journal on Applied Mathematics 47 (1987), no. 2, 232-243.

[27] M. Taki, Homoclinic chaos in bistable optical systems, Proceedings of the International Conference on Nonlinearity, Bifurcation and Chaos: The Doors to the Future (ICNBC'96), Łódź, September 1996, pp. 239-242.

Jan Awrejcewicz: Department of Automatics and Biomechanics, Technical University of Lodz, 1/15 Stefanowski Street, 90924 Lodz, Poland

E-mail address: awrejcew@p.lodz.pl

Mariusz Holicke: Department of Automatics and Biomechanics, Technical University of Lodz, 1/15 Stefanowski Street, 90924 Lodz, Poland 


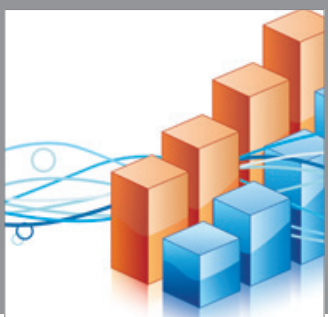

Advances in

Operations Research

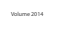

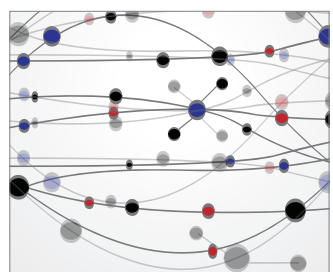

\section{The Scientific} World Journal
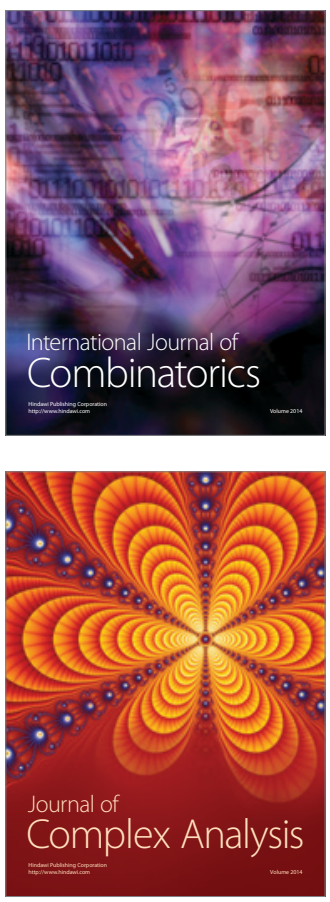

International Journal of

Mathematics and

Mathematical

Sciences
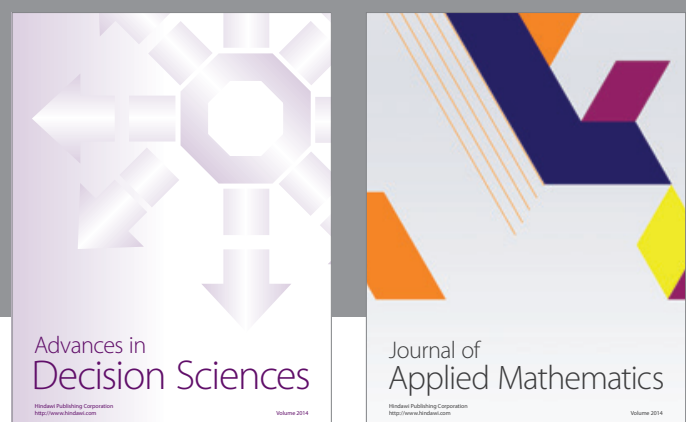

Journal of

Applied Mathematics
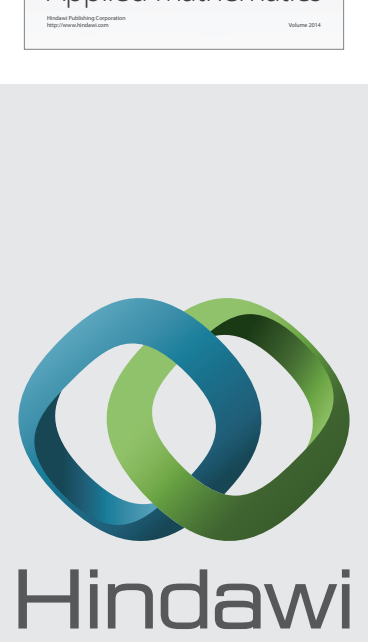

Submit your manuscripts at http://www.hindawi.com
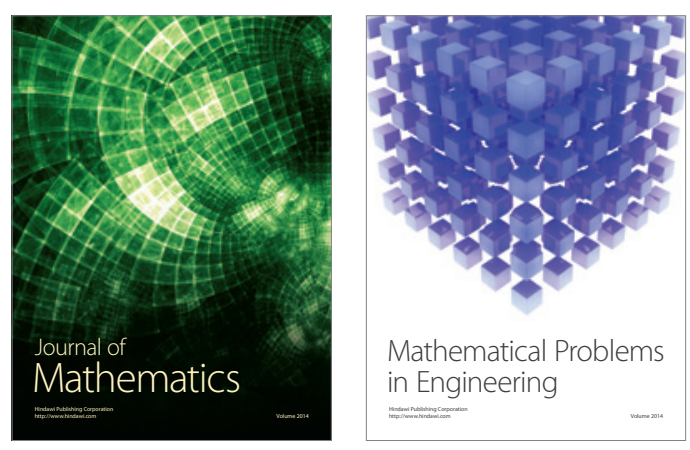

Mathematical Problems in Engineering
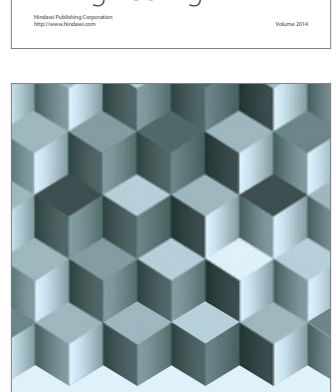

Journal of

Function Spaces
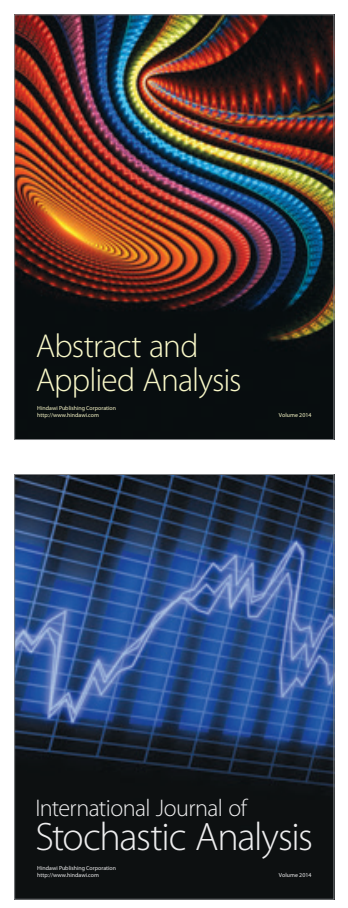

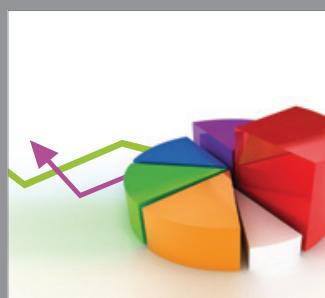

ournal of

Probability and Statistics

Promensencen
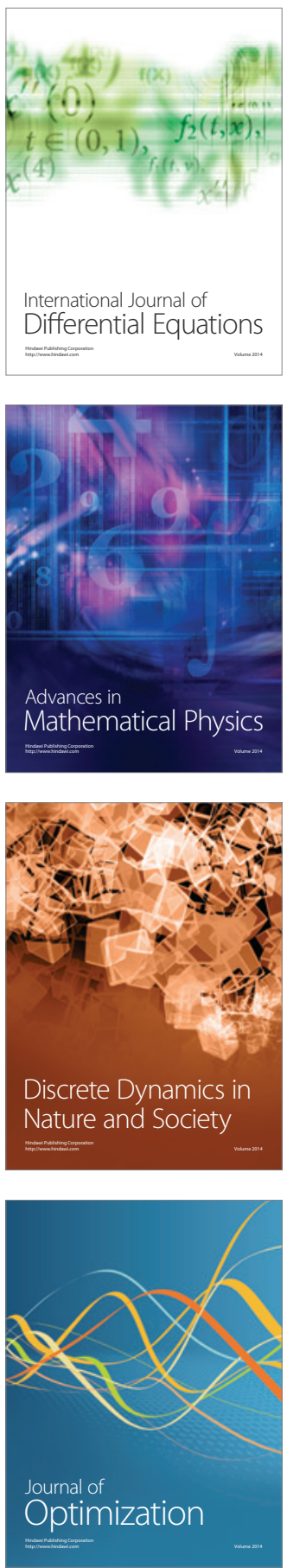